\title{
XV Overall Polish in vitro Culture and Plant Biotechnology Conference
}

\section{Biotechnological utilization of in vitro culture variability}

\author{
September 17-20, 2018, Rogów
}

The conference is dedicated to Prof. dr hab. Maciej Zenkteler

\begin{abstract}
Session I Various faces of biotechnology progress
Session II New tools in plant biotechnology

Session III Haploids and chromosome analysis

Session IV Biotechnology in flora protection

Session V Plant growth regulators and plant cell in stress conditions

Session VI Biotechnology in pharmacology

Session VII Biotechnology application increases the biological knowledge
\end{abstract}

Conference is held under patronage

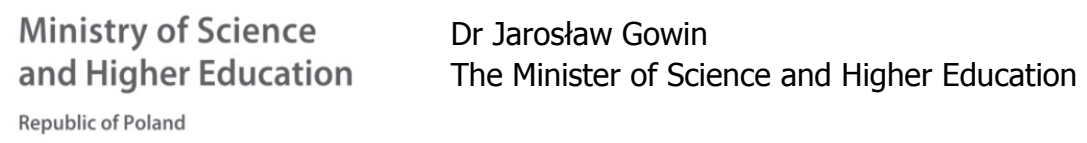

The Rector of Warsaw University of Life Sciences - SGGW

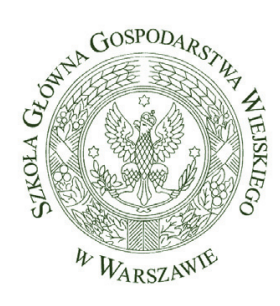

\section{Co-organizers}

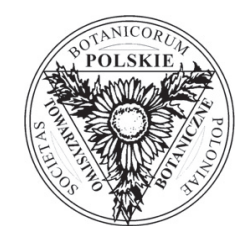

$0 / 2$

POLSKA AKADEMIA NAUK
OGRÓD BOTANICZNY-CENTRUM ZACHOWANIA RÓZZNORODNOŚC ZNEJ W POWSINIE
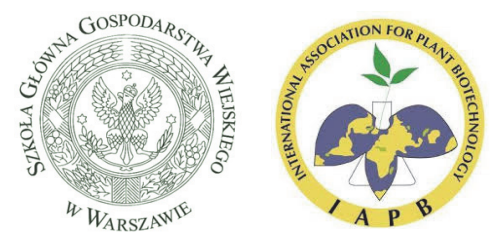
Prof. dr hab. Wiesław Bielawski

Prof. dr hab. Romuald Zabielski

Prof. dr hab. Tomasz Twardowski

Prof. dr hab. Henryk Żybura

Dr hab. Adam Rostański

Dr Paweł Kojs

Prof. dr hab. Jan J. Rybczyński

Prof. dr hab. Teresa Cegielska-Taras

Prof. dr hab. Franciszek Dubert

Prof. dr hab. Małgorzata Gaj

Dr hab. Ewa Hanus-Fajerska

Prof. dr hab. Ewa Kępczyńska

Prof. dr hab. Jan Kępczyński

Prof. dr hab. Ewa Łojkowska

Prof. dr hab. Anna Mikuła

Dr hab. Rafał Mól, prof. UAM

Prof. dr hab. Agnieszka Pietrosiuk

Prof. dr hab. Monika Rakoczy-Trojanowska

Prof. dr hab. Jan Szopa-Skórkowski

Prof. dr hab. Zofia Szweykowska-Kulińska

Dr hab. Barbara Thiem, prof. UM

Prof. dr hab. Tomasz Twardowski

Prof. dr hab. Przemysław Wojtaszek

Prof. dr hab. Halina Wysokińska

Prof. dr hab. Elżbieta Zenkteler

Prof. dr hab. Janusz Zimny

\section{Honoree Committee}

The Rector of Warsaw University of Life Sciences - SGGW

Corresponding Member, Chair of the council of provosts

of Biological and Agricultural Sciences Division II of the PAS

President of Biotechnology Committee PAS

Dean of The Faculty of Forestry,

Warsaw University of Life Sciences - SGGW

President of Polish Botanical Society

Director of PAS Botanical Garden - CBDC in Powsin

\section{Scientific Committee}

President, PAS Botanical Garden - CBDC in Powsin

Plant Breeding and Acclimatization Institute (IHAR) - National

Research Institute, Poznań Division

Institute of Plant Physiology PAS in Crakow

Department of Genetics, University of Silesia in Katowice

President of Plant Tissue Culture Section

of Polish Botanical Society

Department of Plant Biotechnology,

University of Szczecin

Department of Plant Physiology and Genetic Engineering,

University of Szczecin

Department of Biotechnology, Intercollegiate Faculty of Biotechnology University of Gdansk and Medical University of Gdansk

PAS Botanical Garden - CBDC in Powsin

Department of General Botany, Institute of Experimental Biology, Adam Mickiewicz University, Poznań

Department of Pharmaceutical Biology and Medicinal Plant

Biotechnology, Medical University of Warsaw

Department of Plant Genetics, Breeding and Biotechnology,

Faculty of Horticulture, Warsaw University of Life Sciences-SGGW

Department of Genetical Biochemistry, Faculty of Biotechnology, Universisty of Wrocław

Institute of Molecular Biology and Biotechnology,

Adam Mickiewicz University, Poznań

Department of Pharmaceutical Botany and Plant Biotechnology,

Poznan University of Medical Sciences

Institute of Bioorganic Chemistry PAS, Poznań

Institute of Molecular Biology and Biotechnology,

Adam Mickiewicz University, Poznań

Department of Biology and Pharmaceutical Botany,

Medical University of Łódź

Department of General Botany, Institute of Experimental Biology, Adam Mickiewicz University, Poznań

Plant Breeding and Acclimatization Institute (IHAR) - National Research Institute in Radzików

\section{Organizing Committee}

Organizing Committee consists form scientists of Department of Experimental Plant Biology

PAS Botanical Garden - CBDC in Powsin, members of Polish Botanical Society

Prof. dr hab. Anna Mikuła

MSc. Małgorzata Grzyb

Prof. dr hab. Jan J. Rybczyński

Dr Karolina Tomiczak

Dr Aneta Baczewska-Dąbrowska

Dr Marcin Olszak

MSc. Wojciech Tomaszewicz

MSc. Jan Marcinkiewicz

Dr Ewa Muszyńska
President

Secretary

Faculty of Agriculture and Biology Department of Botany, Warsaw University of Life Sciences - SGGW 


\section{Ladies and Gentlemen, Dear Participants, Dear Friends}

Let me dedicate the present Conference to the memory of prof. dr hab. Maciej Zenkteler (1931-2017), professor of Adam Mickiewicz University in Poznań, the great plant tissue culturist and plant experimental embryologist. A great academic teacher of botany, supervisor of Master theses and $\mathrm{PhD}$ dissertations, our mentor and friend. The organizer of the Second and Third Conference held in Poznań, in 1975 and 1980.

Our Conference represents a significant contribution towards better understanding of the progress in plant biotechnology that has been made in nearly forty five years. Today we have been meeting for the fifteenth time since 1974, the year of the first conference organized by dr hab. Jan Stefan Bojanowski and held by the Institute of Plant Breeding and Acclimatization in Radzików. This is the fifteenth Conference and 9 speakers have been invited to talk about the newest trends and latest results of their research groups in the field of plant biotechnology.

To summarize our scientific achievements, I am very proud to say that altogether we have listened to 98 session lectures, 169 oral presentations and have seen 1055 posters presented by over 1700 Conference participants attending. Since the second Conference, Abstract Books have been independently published using the available techniques of printing. The Polish Journal Biotechnologia and its modern edition BioTechnologia initially published in Polish and subsequently in English has helped us to distribute the Conference achievements. The Conference has been hosted in many centers throughout Poland: the Institute of Plant Breeding in Radzików (twice), the Institute of Plant Genetics PAS (twice), the Institute of Pomology in Skierniewice, Warsaw University of Medicine in Warsaw, University of Silesia in Katowice, Agriculture University in Krakow, University of Technology and Agriculture in Bydgoszcz, University of Gdańsk, University of Szczecin, Adam Mickiewicz University in Poznań, University of Life Sciences in Poznań and the Polish Academy of Sciences Botanical Garden - Center of Biological Diversity Conservation in Warsaw (twice). The leading persons, in their present position, in respective Centers are: Doctors Andrzej Stolarz, Maciej Hempel, Janusz Zimny, Professors Elżbieta Zenktler and Maciej Zenkteler, Professors Mirosława Furmanowa, Barbara Skucińska, Lucyna Drozdowska, Mirosław Małuszyński, Ewa Kępczyńska, Jan Kępczyński, Ewa Łojkowska, Anna Mikuła and Zbigniew Broda. I would like to extend my special thanks to numerous members of local Organizing Committees and Scientific Committees for their wonderful job in helping me to carry on forward the idea of the Tissue Culture and Plant Biotechnology in our country. I also wish to express my deep thanks to the Authorities of the all universities and institutes for their assistance and readiness to let us use their premises and facilities of their institutions for the purpose of our Conferences.

Special thanks go to my younger co-workers: prof. dr hab. Anna Mikuła and dr Karolina Tomiczak who have been assisting me and providing support in organizing Conferences since they joined my scientific team.

The program includes the following subjects of interest: new tools in plant biotechnology, in vitro plant differentiation and its conditions, plant cell in stress conditions, utilization of an in vitro culture and plant biotechnology.

Finally, I am very glad to introduce to all of you 101 abstracts of lectures, oral presentation and posters presenting the scientific program of plant tissue culture and biotechnology in Poland today. 


\section{PROGRAMME \\ XV Overall Polish in vitro Culture and Plant Biotechnology Conference \\ September 17-20, 2018, Rogów}

\section{Monday September $17^{\text {th }}, 2018$}

15:00 Registration and poster mounting

18:00 Welcome Party

Tuesday September $18^{\text {th }}, 2018$

7:30-8:30 Registration

\section{8:30 Open Ceremony (Chairman: prof. Anna Mikuła and prof. Jan J. Rybczyński) \\ 8:45 JAN J. RYBCZYŃSKI \\ Forty four years of activity and fifteenth conference}

9:00 Session dedicated to the memory of prof. dr hab. Maciej Zenkteler

9:00 Rafat Mól

Scientific achievements of the holy memory Professor Maciej Zenkteler (1931-2017)

\section{9:30 Session I. Various faces of biotechnology progress}

(Chairman: prof. Elżbieta Zenkteler)

9:30 IwONa Kosieradzka, MAGdalena MATUSIEWICZ

The use of genetically modified organisms in the diet of humans and animals

10:00 FrankLin Gregory

Plant recalcitrance against Agrobacterium mediated transformation - present status and future perspectives

\section{0:30 Coffee break}

10:50 Aleksandra Królicka, Marta Krychowiak, Angelika MichalaK, Daria Świgoń, Weronika GidZińSKa, Rafat BanasiUK, AGNIESZKa CHYLEWSKA, MAGDALENA ZiąBKA, MAGDALENA NARAJCZYK Plants and nanoparticles of metals - perspectives and challenges

11:20 Tomasz Skrzypczak, Shraddha Wadurkar, Rafat Krela, WojCieCh KWiatKowski, Mostafa Wahby, Stig NielSEN, Mary K. Heinrich, Payam Zahadat, Daniel Hofstadler, IgOr KuKSin, Kasper Stoy, Serge Kernbach, Thomas SChmickL, Phil Ayres, Heiko Hamann, Przemystaw Wojtaszek Plants stimulation for development of flora robotica bio-hybrid

11:50 Stawomir Sowa, ANNA LinKIEWICZ, JANUSZ ZimnY Detection, identification and quantification of products resulting from the use of new plant breeding techniques

12:20 Session II. New tools in plant biotechnology (Chairman: prof. Andrzej Joachimiak)

12:20 WaCkaw OrCZYK, Sebastian GaSParis, AnNa Nadolska-OrczYK Genome editing - new frontiers in plant biotechnology and plant breeding

\section{2:50 EWA KĘPCZYŃSKA}

Regulation of somatic embryogenesis induction in Medicago truncatula Gaertn. at the physiological and molecular level

13:20 ANNa Wójcik, MatgoRzata Gaj

microRNA - small molecules with a huge impact on the regulation of developmental plasticity of plant somatic cells cultured in vitro

\section{3:50 Lunch}

\section{4:50 Continuation of session II}

14:50 Magdalena ŻuK, Jan SZOPa-SkóRKOWSKi Techniques of gene function assessment using GMO and EMO methods

15:20 Sebastian Gasparis, Maciej Kata, Mateusz Przyborowski, WaCŁaw Orczyk, AnNa Nadolska-Orczyk Genome editing in barley - application in gene function studies and modifications of useful traits

15:35 Karolina Sobańska, Joanna Cerazy-WaliszeWSKa, Aurelia ŚluSarkieWicz-JARZina, Aleksandra PonitKa, STANISŁAW JEŻOWSKI, TOMASZ PNIEWSKI Biolistic transformation of Miscanthus sinensis and Miscanthus $\times$ giganteus

15:50 EMILIA MichatowsKa, JUSTYNA LEMA-RUMIŃSKA Evaluation of ISSR markers usefulness in the research of Chrysanthemum x grandiflorum Ramat./Kitam. plants obtained via somatic embryogenesis 


\section{6:05 Alicja Tymoszuk}

Silver and cooper nanoparticles in iv vitro culture of chrysanthemum: disinfecting properties and influence on adventitious shoots regeneration

16:20 Magdalena DZiato, Jan SZOPA-SKóRKOWSKI, Magdalena ŻUK Directed and hereditary modification in CHS gene methylation induced by oligonucleotides

16:35 First poster session and coffee break

18:30 Dinner

19:30 Workshop

Wednesday September $19^{\text {th }}, 2018$

8:30 Session III. Haploids and chromosome analysis (Chairman: prof. Anna Mikuła)

8:30 JANUSZ ZIMNY

Induced rye embryogenesis - a summary of 35 years of research

9:00 IwONA ŻuR

Activity of an antioxidative system as a factor determining microspore embryogenesis in triticale (x Triticosecale Wittm.)

9:30 Edyta Skrzypek, Kinga DZiurka, Ilona CzyczyŁo-Mysza, IZABela MarcińSKa, Kamila KapŁoniaK, ANGELIKA NOGA, MARZENA WARCHOK

Production of oat (Avena sativa L.) doubled haploids via androgenesis and wide crosses

10:00 SyLWia OleszCZUK

Meiotic restitution as a process determining doubling the chromosome number in haploids of triticale

10:30 ANDRZEJ JOACHIMIAK

Plant sex chromosomes in vivo and in vitro

11:00 Coffee break

\section{1:20 Continuation of session III}

11:20 Marzena Warchot, Edyta SKRZYPeK, Ilona CZyCZYŁo-Mysza, Kinga DZiURKa, IZabela MarcińSKa, ANGELIKA NOGA, KAMILA KAPŁONIAK

Oat (Avena sativa L.) anther culture: the effect of pretreatment and media composition on embryo-like structures production

11:35 Monika Gajecka, Marek Marzec, Beata Chmielewska, Janusz Jelonek, Justyna Zbieszczyk, Iwona Szarejko Chloroplast differentiation during regeneration of green and albino plantlets in microspore culture of barley

11:50 Dorota Weigt, Sylwia Mikotajczyk, Janetta Niemann, AgnieszKa Tomkowiak, Danuta Kurasiak-Popowska, JOANNA ZYPRYCH-WALCZAK, IDZI SIATKOWSKI

The effect of zearalenone on the anther culture of wheat

12:10 Session IV. Biotechnology in flora protection (prof. Janusz Zimny)

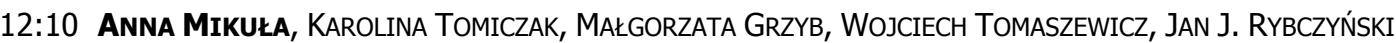
Biotechnology in plant diversity conservation

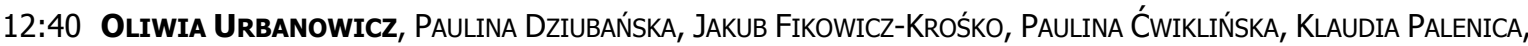
MAGDALENA BAJOR, ZOFIA OZYMKO Large scale micropropagation of rare and endangered mosses

12:55 Marzena Parzymies, Magdalena Pogorzelec, Barbara Banach-AlbińSKa, Alicja Świstowska, AGNIESZKA SZCZUROWSKA, ARTUR SERAFIN Use of tissue culture for ex situ conservation of the endangered Salicaceae species in the territory of Poland

13:10 ANNA ŹRÓBEK-SOKOLNIK Is it possible to regenerate Pulsatilla patens (L.) Mill. from petioles using in vitro cultures?

13:25 Bożena Szewczyk-Taranek, Barbara Prokopiuk, Monika Cioć, Bożena PaWŁowska Cryopreservation of shoot tips serves Staphylea pinnata L. germplasm conservation

13:40 Lunch

14:40 Session V. Plant growth regulators and plant cell in stress conditions (Chairman: prof. Jan Szopa-Skórkowski)

14:40 JAN KĘPCZYŃSKI Smoke-derived karrikin - a new natural compound regulating growth and development of plants

15:10 Karolina Nowakowska, AndRZej PaCholczaK Meta-Topolin as an alternative to 6-Benzyladenine during micropropagation 
15:25 IwONA SZYP-BOROWSKa, KRYSTYNA SZCZYGIEt, JAN KOWALCZYK

In vitro regeneration of adult Pinus sy/vestris $L$. trees

15:40 Agnieszka PŁażek, Aneta SŁomka, Franciszek Dubert, PrzemysŁaw Kopeć, marta Hornyák, MICHAK DZIURKA, JAKUB PASTUSZAK

The effect of trophic stress on embryo sac development in pistils of Fagopyrum esculentum (Moench.) in in vitro conditions

16:10 Aleksandra Boba, Jan SZOPA-SKÓRKOWSKI, ANNA KULMa Terpenoids in flax response to fusariosis

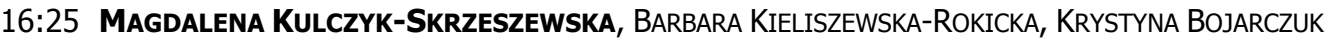
Inoculation of Populus nigra 'Italica' and P. $x$ canescens microplants with mycorrhizal fungi increase their tolerance to salt stress

16:40 Weronika Kozłowska, Kamil WiŚnieWSKI, NATALIA TRETER, AdAM MatKowSKi Effect of photosynthetically active radiation (PAR) and white $L E D$ light on growth and rosmarinic acid production of Perovskia spp. in vitro cultures

\section{6:55 Coffee break}

\section{7:15 Continuation of the session $V$}

17:15 Barbara Piwowarczyk, Krzysztof Tokarz, Wojciech Makowski, Izabela WyŁuPek Comparison of grass pea seedlings response to osmotic and chemical stress in in vitro conditions

17:30 DARIUSz KuLUS

Molecular, biochemical and phenotypic evaluation of Lamprocapnos spectabilis (L.) Fukahura plantlets recovered from shoot tips cryopreserved via encapsulation-vitrification technique

\section{7:45 Second poster session}

19:30 Gala dinner

\section{Thursday September $20^{\text {th }}, 2018$}

\section{8:30 Session VI. Biotechnology in pharmacology} (Chairman: prof. Ewa Kępczyńska)

\section{8:30 Ewelina Piatczak}

Iridoid and phenylethanoid glycosides accumulation in in vitro cultures of plants from Rehmannia genus

9:00 Katarzyna Mańko, Mateusz Kawka, Katarzyna SykŁowska-Baranek, Maciej Pilarek, Agnieszka Pietrosiuk A simultaneous root immobilization and in situ extraction for intensification of secondary metabolism in Rindera graeca in vitro cultures

\section{9:15 BARBARA THIEM}

Application of in vitro culture techniques in the maintenance of Linnaea borealis $L$. - protected species of Polish flora

9:30 AnNa Stojakowska, NatAlia KŁECZEK, JANUSZ MaLARZ Biologically active terpenoid constituents from shoot cultures of Carpesium divaricatum

\section{9:45 Coffee break}

10:05 Session VII. Biotechnology application increases the biological knowledge (Chairman: prof. Małgorzata Gaj)

10:05 Marcin Olszak, Petr Berko, Wojciech Burza Induced totipotent stem cells and somatic embryogenesis in plants: new answers to old questions

10:35 Laurencja Szata, Katarzyna Sosnowska, Teresa Cegielska-Taras Biotechnological methods used in Brassica napus resynthesis

11:05 Wojciech SZYPUŁA, AgnieSZKa Pietrosiuk In vitro culture and biotechnology of Lycopodiaceae sensu lato. Have hopes been fulfilled?

11:35 EWA KACZMAREK, MAŁGORZATA PAŁUCKA DNA barcoding and banking as a way to preserve forest biodiversity in the Białowieża Forest, Poland

11:50 Matgorzata GrzYb, JAN JAROSŁAW RyBCZYŃSKI, ANNA MikUtA Single- versus multiple-cell origin of somatic embryos in the tree fern Cyathea delgadii

12:05 JAN JAROSŁaW RYBCZYŃSKI, KaROLINA TOMICZAK, ANNA MiKUŁA Various levels of the morphogenic phenomenon in tree ferns in vitro culture

\section{2:35 Summary of the conference - achievements}

13:00 Lunch and good by for every one 


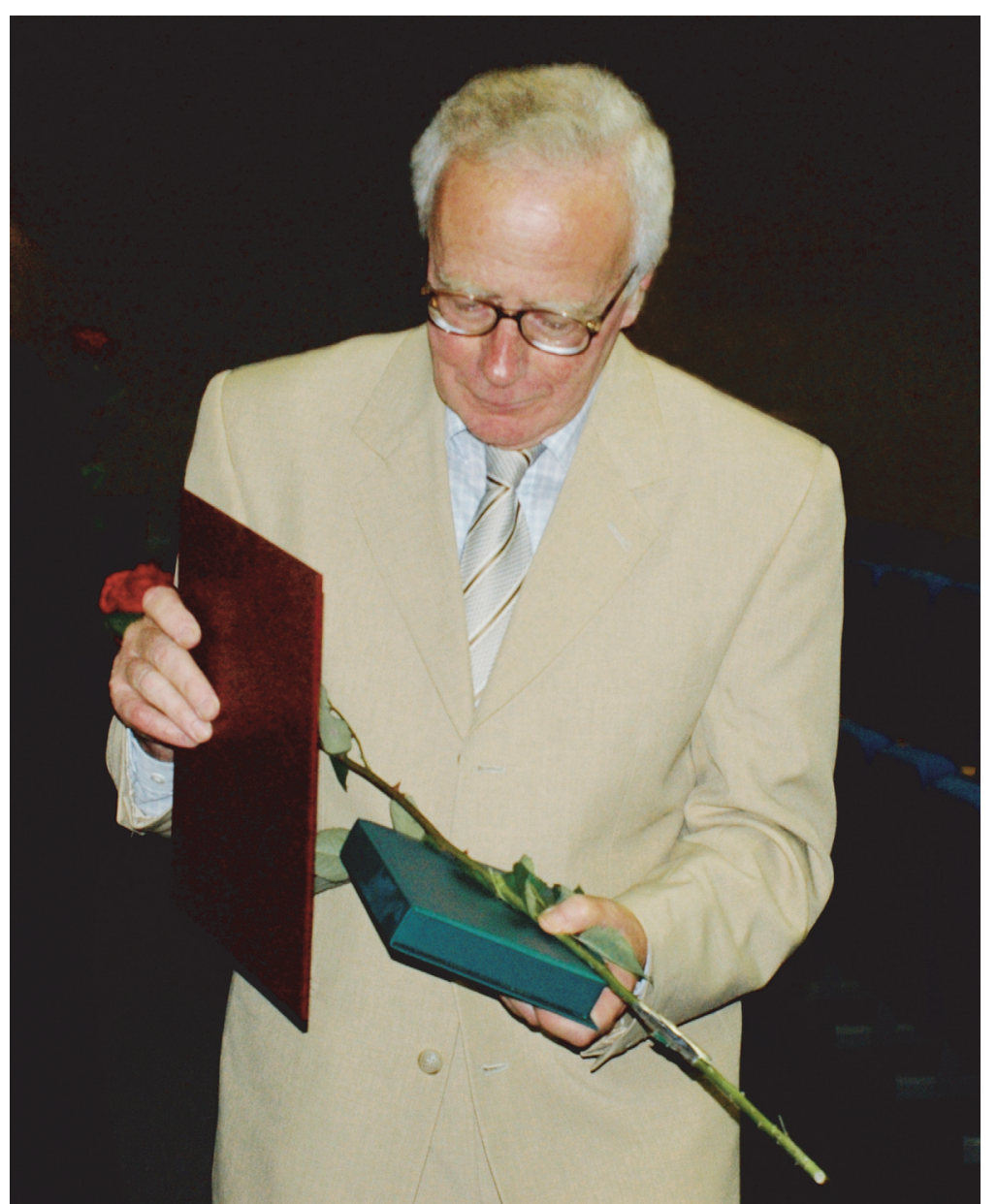

Prof. dr hab. Maciej Zenkteler (1931-2017) 


\section{Hymn na eksperymentowanie Prof. dr hab. Macieja Zenktelera}

Chojnie mikrosporami, pyłkami i zalążkami szafował, steryliami zawsze materiał roślinny traktował.

Pąki kwiatowe różnie kokietował, no i nawet je kastrował,

na kwiaty i kłosy izolatory zakładał.

Słupki wycinał, zalążnie rozcinał,

worki pyłkowe natomiast przecinał.

Łożysko izolował,

łagiewki ku woreczkom zalążkowym zaganiał, mezalians aranżował,

niańki dla zarodków angażował.

Mikro- i makroelementy hojnie rozlewał, cytokininami i auksynami mniej obficie polewał, umiarkowanie pożywki słodził, natomiast pylniki głodził,

światłem lub ciemnością reakcje ich indukował, chłodem lodówkowym również traktował, chromosomy ostatecznie zredukował.

Obserwował, wybierał, materiał utrwalał, kroił go i barwił

i wszystkich pięknymi fotografiami karmił,

W tych działaniach pięćdziesiąt pięć lat On był stały, tylko one się zmieniały.

Jan J. Rybczyński

\section{Anthem to Professor Maciej Zenkteler's experimenting}

He squandered generously with microspores, pollen and embryos, always sterilising the treated plant material promptly.

Flower buds he coquetted variously, and well, sometimes he castrated them, too. and also put insulators on flowers and ears.

He cut out pistils, and cut open ovaries,

while cutting through the pollen sacks as well.

Then he would insulate the placenta, drive the pollen tubes towards embryo sacs, arranging mesalliance,

and then engaged nannies for the embryos.

He generously poured out micro- and macronutrients, supplementing them less heavily with cytokinins and auxins, and moderately sweetened the media, starving, in the meantime, the anthers.

He induced their reactions with light and darkness, but also treated them with fridge cold, eventually reducing chromosomes.

He then observed, selected, and recorded the material, sliced it and stained,

and finally treated everyone to beautiful photographs.

For fifty five years he continued to do so, persistent in his activities, while only species were changing over time. 


\title{
Scientific achievements of the holy memory Professor Maciej Zenkteler (1931-2017)
}

\author{
RAFAŁ MóL \\ Department of General Botany, Faculty of Biology, Adam Mickiewicz University, Poznań, Poland \\ *ramol@amu.edu.pl
}

Maciej Zenkteler (1. 10. 1931 - 6. 08. 2017) pursued his academic career at the Adam Mickiewicz University in Poznań where he completed the studies in biology and started to teach in 1956 . He graduated in 1957 with a master's degree in botany working on the root tissue culture of carrot. Then he continued studies on male sterility in carrot which also earned him his $\mathrm{PhD}$ degree in 1962. He became a world-known scientist in experimental embryology thanks to his works on in vitro pollination of ovules and embryo rescue in distant crosses of angiosperms. In this field of research, he completed his habilitation procedure in 1968, and became a professor in 1978 and finally the professor ordinarius in 1989. He was head of the Laboratory of General Botany in 1978-2002. When retired, he continued to work on intergeneric pollination in vitro, and haploid or hybrid plant development. This was the main area of interest over his entire life but he also worked on androgenesis in vitro and the plant micropropagation as well. Among Professor's most important discoveries were fertilization analyses in interspecific and intergeneric crosses in Caryophyllaceae family, haploid embryo development after crossing wheat with Hordeum bulbosum, induction of androgenesis in anther cultures of solanaceous plants and grasses, obtaining of Salix $\times$ Populus hybrids by ovule pollination in vitro and embryo rescue. Professor Maciej Zenkteler had over 90 scientific papers published, and he was the editor and co-author of a unique textbook for Polish students and scientists interested in plant in vitro techniques Hodowla komórek i tkanek roślinnych (Plant Cell and Tissue Culture). However, not only his personal scientific achievements are important. Even more important was how he has influenced his students, colleagues and co-workers. Professor supervised Master theses of 126 students and $9 \mathrm{PhD}$ dissertations and reviewed $44 \mathrm{PhD}$ dissertations, 23 works for postdoctoral degrees, and 14 for professor's titles. He collaborated with many scientific institutions in Poland where he initiated new research in relation to haploid production and wide hybridization. Professor Maciej Zenkteler was deeply involved in research projects at the institutes of the Polish Academy of Sciences: the Institute of Plant Genetics in Poznań, the Institute of Dendrology in Kórnik, as well as at the Plant Breeding and Acclimatization Institute (IHAR) - National Research Institute in Radzików, and IHAR Research Centers in Poznań and Bydgoszcz. He was always ready to discuss problems and to suggest fascinating research topics. By his work and experience in plant tissue cultures and plant embryology, he was very well recognized and often invited by departments or laboratories using in vitro techniques for experimental embryology in Poland (to mention only the Jagiellonian University and the Agricultural University in Kraków, the Maria Skłodowska-Curie University in Lublin, the Botanical Gardens in Powsin, Poznań University of Life Sciences, the Institute of Plant Physiology in Kraków), and also abroad (e.g. Max-Planck-Institutes in Tübingen and Köln, Freie Universität in Berlin, Ghent University). He was often invited to give talks at many European universities and at numerous scientific conferences around the world. Professor Maciej Zenkteler, his life and work, had a great impact on the development of a new research branch - experimental embryology, and on the implementation of new methods in crop breeding and plant production in Poland. 


\section{Sponsors}

OLMPUS Olympus Polska Sp. z o.o.

\section{(hiogenet) \\ WYPOSAŻENIE LABORATORIUM}

\section{$\Rightarrow$ A.G.A. \\ Analytical}

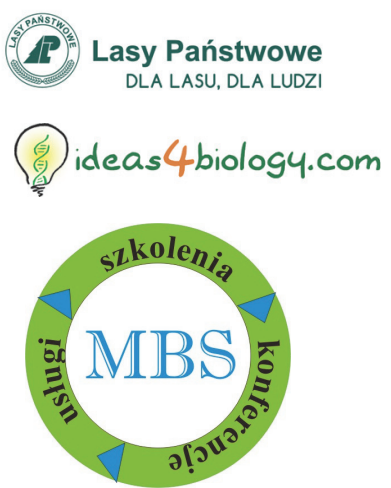

Biogenet Sp. z o.o.

A.G.A. Analytical

State Forests

ideas4biology

MBS - Szkolenia, Konferencje, Usługi Sp. z o.o.

\section{Media patronage and partners}

类

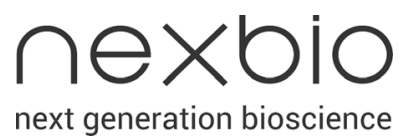




\title{
The use of genetically modified organisms in the diet of humans and animals
}

\author{
IWONa Kosieradzka *, Magdalena Matusiewicz \\ Department of Animal Nutrition and Biotechnology, Faculty of Animal Science, Warsaw University of Life Sciences - SGGW \\ Warszawa, Poland \\ *iwona_kosieradzka@sggw.pl
}

Despite the constantly growing acreage of cultivation of GM plants and over 20 years long history of GM crops utilization in feeds and foods, the safety of their influence on animal and human health is still a subject of hot public debates and various anti-GMO campaigns.

The resistance to biotic and abiotic factors limiting the production of GM plants can be an important contribution to solving the growing problem of the global food deficit, increasing the stability of production. It seems, however, that also biofortification of the diet through the genetic modification of plant raw materials accumulating high quality nutrients, micronutrients and bioactive substances is a strategy worth considering both in the context of the phenomenon of so-called "hidden hunger" and to meet the specific needs of highly developed societies in Europe. GM crops can be a source of deficient nutrients, substances with detoxifying, immunomodulatory, anti-carcinogenic, therapeutic proteins, a component of special diets dedicated to consumers with health problems (e.g. those suffering from allergy) or have particularly desirable sensory characteristics. Vegetable GM feedstocks may have features that facilitate storage. The use of GM plants for feed purposes creates the opportunity of influencing the productivity and health of animals, improve the quality of animal products, and reduce the harmful effects of animal production on the environment.

Despite the potentially wide range of applications of GMOs in human and animal nutrition, they are not fully accepted in by consumers and producers of food and feed in EU Member states. Raw materials obtained by cisgenesis and intragenesis are more favored than those obtained via transgenesis. It seems that "New breeding techniques" (or new breeding innovations - Zinc finger nuclease (ZFN) technology, CRISPR systems Oligonucleotide directed mutagenesis (ODM), RNA-dependent DNA methylation (RdDM), Grafting, Cisgenesis and intragenesis, Synthetic genomics, etc.) will in the future be more widely used in plants with increased nutritional and dietary value, although their legal status requires regulation.

Regardless of the direction, purpose, technique of modification, the commercialization of consumer crops obtained by genetic modification techniques requires the estimation of risk, which requires in-depth research (case by case) conducted in this direction with the use of advanced laboratory techniques. The analysis of the results of experiments carried out by foreign and national scientific centers (the Institute of Animal Production, the Institute of Genetics and Animal Husbandry PAS, and others), proves the lack of unequivocal, reliable evidence of the negative impact of GMOs authorized for use in animal and human nutrition. The research of genetically modified plants shows, for the most part, no significant differences in relation to raw materials not modified and in many cases indicates the benefits resulting from their use in the diet.

The assessment of the intended and unexpected effects of modifications in the context of their nutritional safety and their impact on the consumer's body was also the general goal of the research conducted by the team of the Department of Animal Nutrition and Biotechnology of the Faculty of Animal Science at WULS-SGGW. The studied plants were grown in the laboratories of the Faculty of Horticulture, Biotechnology and Landscape Architecture of the Warsaw University of Life Sciences and the Faculty of Biotechnology of the University of Wroclaw. Experiments on model animals showed no negative impact on the body and a number of benefits of their use as a component of a diet with health effects. 


\title{
Plant recalcitrance against Agrobacterium mediated transformation - present status and future perspectives
}

\author{
FRANKLIN GREGORY \\ Institute of Plant Genetics of the Polish Academy of Sciences, Poznań, Poland \\ *fgre@igr.poznan.pl
}

Agrobacterium is popularly known as the "natural genetic engineer" because of its innate capacity to infect plants and introduce a piece of DNA (T-DNA or transferred DNA) from its tumor inducing (Ti) or root inducing (Ri) plasmid into plant cells via a process known as "T-DNA transfer". Once inside the plant cell, the T-DNA is transported into the nucleus, where it stably integrates into the plant genome. T-DNA encodes genes for the synthesis of auxins, cytokinins, and opines. Hence, T-DNA integration into the host genome and its expression results in an imbalance of host cell auxin/cytokinin ratio, which leads to uncontrolled cell division resulting in the development of "crown galls" or "hairy roots" coupled with opine synthesis. While these neoplastic diseases affect the growth and productivity of the host plants and considered as a disease (Gelvin, 2009), the T-DNA transfer process has been exploited in such a way that Agrobacterium is used as a vector to introduce genes into plants (Nester, 2015).

Today, A. tumefaciens is an indispensable tool in contemporary functional genomics and crop improvement. In spite of the routine use of $A$. tumefaciens in functional genomics of model plant species, several economically important plant species (for example: members of legumes, cereals, biofuel crops, fruit trees, medicinal plants) remain resistant or difficult to transform using this bacterium, due to "plant recalcitrance" a phenomenon not well understood so far. Our ability to apply contemporary molecular biology tools (CRISPR/Cas9, RNA interference: RNAi) is currently limited to a few plant species due to prevalence of this phenomenon across plant kingdom.

Although the mechanism of plant recalcitrance is ambiguous, activation of plant defense is considered to be a prevailing cause (Franklin et al., 2008; Pitzschke, 2013; Hou et al., 2016). When co-cultivated with A. tumefaciens, Hpericum perforatumcells initiated typical defense responses characterized by a biphasic reactive oxygen species (ROS) burst, secondary metabolic changes, cell wall lignification, pathogenesis related $(P R)$ gene upregulation etc., subsequently leading to the mortality of the bacterium, thus preventing T-DNA transfer (Franklin et al., 2007; Franklin et al., 2008, 2009; Singh et al., 2014). On the other hand, A. tumefaciens co-cultivation with tobacco BY2 cells under the same conditions lead to successful T-DNA transfer (Franklin et al., 2008). It is emerging that in both compatible and incompatible plant- $A$. tumefaciens interactions, an initial defense response is induced. In the case of compatible interactions, despite the initial transient activation of basal host defense, the subsequent transfer of virulence factors might lead to the suppression of immune responses, resulting in successful transformation as observed in tobacco (Veena et al., 2003; Franklin et al., 2008). By contrast, for incompatible interactions the initially evoked plant defense response is long lasting (and successful), thereby, affecting the bacterium and preventing T-DNA transfer into plant cells, as observed in $H$. perforatum (Franklin et al., 2008), making these plants recalcitrant to A. tumefaciens - mediated transformation.

The current status of our understanding of the mechanisms of plant recalcitrance against $A$. tumefaciens mediated plant transformation and the future perspectives will be discussed in the light of $A$. tumefaciens $-H$. perforatum interaction. 


\title{
Plants and nanoparticles of metals - perspectives and challenges
}

\author{
AleKSandra Królicka ${ }^{1 *}$, Marta KrychowiaK ${ }^{1}$, ANGElika MichalaK ${ }^{1}$, Daria ŚWigon ${ }^{1}$, \\ WERONIKa GIDZińSKa ${ }^{1}$, RAFAE BANASIUK ${ }^{1}$, AgNIESZKa ChYLEWSKa ${ }^{2}$, \\ MAGDALENA ZIABKA ${ }^{3}$, MAGDALENA NARAJCZYK ${ }^{4}$ \\ ${ }^{1}$ Laboratory of Biologically Active Compounds, Intercollegiate Faculty of Biotechnology University of Gdansk \\ and Medical University of Gdansk, Gdańsk, Poland \\ ${ }^{2}$ Laboratory of Intermolecular Interactions, Faculty of Chemistry, University of Gdansk, Gdańsk, Poland \\ ${ }^{3}$ Department of Ceramics and Refractories, Faculty of Materials Science and Ceramics, \\ AGH University of Science and Technology, Kraków, Poland \\ ${ }^{4}$ University of Gdansk, Faculty of Biology, Laboratory of Electron Microscopy, Gdańsk, Poland \\ *aleksandra.krolicka@biotech.ug.edu.pl
}

Nanotechnology is a fast-growing field of science due to the increasing interest in nanostructures. Nanoparticles are nanostructures with at least one dimension under $100 \mathrm{~nm}$ and a high surface to mass ratio. Silver nanoparticles are the most popular type of nanomaterials. Because of their antimicrobial properties, they found various applications in a number of products: medical, personal hygiene, cleaning and disinfection, building materials, textiles, and even food processing. Silver nanoparticles are produced mainly by chemical synthesis. However, chemical approaches are based on the use of potentially hazardous substances. Thus, more research is concentrated on alternative production methods. One of them is a synthesis with the use of plant extracts. Biomolecules that are present in those extracts usually act as both reducing and capping agents. Moreover, many of extracts' components have shown to be active against animal and plant pathogens on their own. Green synthesis is energy efficient, easy, and fast. It allows to avoid the use of toxic and dangerous compounds so it does not harm the environment. In this method different biological systems, including bacteria, fungi, and plants, are used for the production of nanoparticles. Biological synthesis methods allow to control the size and shape of nanoparticles. These are important factors considering their biomedical use. Silver nanoparticles have already been synthesized using plant extracts from: Magnolia grandiflora, Eucalyptus angophoroides, Citrus limon, Capsicum annuumL., Camellia sinensis, Cinnamomum altissimum Kosterm., Rosa rugosa, Hibiscus cannabinus, Cycas, Acalypha indica, Coffea L., Drosera sp., and many others. The antimicrobial properties of the silver nanoparticles obtained using the extracts of the aforementioned plant species are very often comparable to nanoparticles obtained by the chemical method. Depending on the extract used for the nanostructure synthesis and experimental conditions, a different antimicrobial activity of the final product can be obtained. Because of the multiplicity of factors which may influence the activity of silver nanoparticles new and more efficient ways of their synthesis are still being investigated. An excellent example of the use of green chemistry is the production of silver nanoparticles utilising carnivorous plants (Drosera sp and Dionaea muscipula). Carnivorous plants are a natural source of pharmacologically important compounds (e.g. glucosides, flavonoids, phenolic acids) that are used as substrates in the production of pharmaceuticals with a great antimicrobial potential against Gram positive and Gram negative bacteria. Our results show that flavonoids from carnivorous plants possess high antioxidant properties and thus are also excellent silver ions bioreductans. In addition, silver nanoparticles obtained using carnivorous plants can be combined with secondary metabolites isolated from plants or whole extracts from plant material. This combination successfully creates a synergistic effect which allows to reduce the doses of both components in order to fight human pathogens, which leads to a decrease in the cytotoxicity of both silver nanoparticles as well as secondary metabolites, and thus ensures safety conditions? for potential future patients. 


\title{
Plants stimulation for development of flora robotica bio-hybrid
}

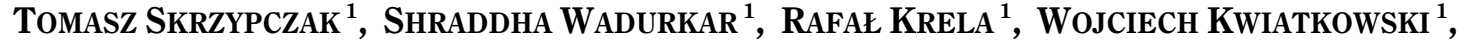 \\ Mostafa Wahby $^{2}$, Stig Nielsen ${ }^{3}$, Mary K. Heinrich ${ }^{4}$, Payam Zahadat $^{5}$, Daniel Hofstadler ${ }^{5}$, \\ Igor Kuksin ${ }^{6}$, Kasper Stoy ${ }^{3}$, Serge Kernbach ${ }^{6}$, Thomas Schmickl ${ }^{5}$, Phil Ayres ${ }^{4}$, Heiko Hamann ${ }^{2}$, \\ PrzemyseaW WOJTASZEK ${ }^{1 *}$ \\ ${ }^{1}$ Department of Molecular and Cellular Biology, Adam Mickiewicz University, Poznań, Poland \\ ${ }^{2}$ Universität zu Lubeck, Lubeck, Germany \\ ${ }^{3}$ IT University of Copenhagen, Copenhagen, Denmark \\ ${ }^{4}$ Centre for Information Technology in Architecture (CITA), Copenhagen, Denmark \\ ${ }^{5}$ Universität Graz, Graz, Austria \\ ${ }^{6}$ Cybertronica UG GmbH, Stuttgart, Germany \\ *przemow@amu.edu.pl
}

Flora robotica in an interdisciplinary project that focus on development of plants-robots bio-hybrids that grow together as architectural artifacts. Here we report on diverse achievements that are to be integrated soon into one system. Crucial parts of the designed system are climbing plants, braided artificial structure, controllers for artificial structure and plants. Common bean, Fallopia aubertii and Wisteria sinensis are the species of choice that are being tested for response for different stimulus for plants shaping. We have been working on vibrations, chemical growth regulators and light of different wavelengths as plants stimulation, which are being implemented into the system. Plants growth controller in response to changing light direction have been developed and, respectively. Vascular Morphogenesis Controller (VMC) for growth of braided structures. VMC will provide plants-supporting growth of artificial structures. Recently, Integrated Growth Projection has been made that will help in running reality gap experiments with flora robotica system. Robots required for implementing controllers algorithms are also being constructed. Except robots for actuating plants, also Phytosensor device for getting data from both components will be installed and linked to communicate with actuating robots. In result strongly integrated managed by robots growth of plants and braided structures will be created and form architectural artifacts.

This work is funded by EU Horizon 2020 Future and Emerging Technologies funds under grant agreement No. 640959 . 


\title{
Detection, identification and quantification of products resulting from the use of new plant breeding techniques
}

\author{
SŁaWomir Sowa *, ANNa LinkiEWICZ, JaNuSZ ZimNY \\ Department of Plant Biotechnology and Cytogenetics, Plant Breeding and Acclimatization Institute - National Research Institute, \\ Radzików, Poland \\ *s.sowa@ihar.edu.pl
}

Plant breeding is one of the key elements of the modernization of agriculture towards sustainable food, feed and fuel production. To achieve this goal new biotechnological techniques and especially new plant breeding techniques (NBTs) are continuously being developed. NBTs are a set of innovative methods used for genome improvement (intragenesis, cisgenesis, RNA-dependent DNA methylation, oligonucleotide directed mutagenesis, reverse breeding, zinc finger nucleases ZFN1-3, TALE nucleases, CRISPR-Cas9 system). The current legal definition of GMOs was elaborated when these techniques where not discovered so it is unclear which of them should fall under the GMO legislation. In the European Union (EU) a contained use of genetically modified microorganisms and genetically modified organisms, as well as placing GMOs on the market requires prior authorization. The development and validation of GMO determination methods are, beside GMO risk assessment, an essential part of GMO authorisation in the EU. Current EU legislation requires identification of any authorised GMO and quantification of GMOs authorised as food or fodder. Placing GM varieties on the EU market requires also post market environmental monitoring (PMEM). Robust molecular methods for detection, identification and quantification of GMOs must be developed to monitor GMOs as well as for proper labelling of GM food and feed. These methods must be specific to identify each GMOs ("event specific methods") and meet defined parameters for validation. In this work we present and discuss the possibility of application of molecular methods (Polymerase Chine Reaction - PCR, real-time PCR, digital PCR, New Generation Sequencing, enzyme-linked immunosorbent assay - ELISA) for detection, identification and quantification of products resulting from the use of NBTs taking into account the level of introduced changes into the plant genome (DNA deletion, DNA insertion, single nucleotide polymorphism etc.). According to some opinions the application of genome editing techniques should not lead to products classified as GMOs in case the of DNA deletion of any size, however it is not clear which changes introduced into genomic DNA by genetic engineering that go beyond single base pair substitution should fall under the GMO legislation. The European Commission is currently investigating the legal status of NBTs. Many breeding companies have already developed new varieties of agricultural plant species with the use of NBTs but in the situation of the lack of legal clarity, they are not placing them on the market yet. Scientists are also routinely implementing these new techniques into their research as some of them allow very precise site specific genome editing. Nevertheless, global harmonization of both legal classification of NBTs and the development of methods for GMO determination is a prerequisite for safe implementation of NBTs into plant breeding. It is also crucial for post market environmental monitoring and detection of unauthorized GMOs in the global trade. This is especially important in the current situation where asymmetric and asynchronous authorization of GMOs is becoming a significant problem. 


\title{
Genome editing - new frontiers in plant biotechnology and plant breeding
}

\author{
WaClaw OrczyK *, Sebastian GaSParis, AnNa Nadolska-OrczyK \\ Department of Genetic Engineering, Plant Breeding and Acclimatization Institute - National Research Institute, Radzików, Poland \\ *w.orczyk@ihar.edu.pl
}

A targeted modification of selected loci of a higher plant genome remains a subject of a long term effort. Subsequent generations of targeted mutagenesis methods such as meganucleases, zinc finger proteins, transcription element like proteins and recently CRISPR/Cas9 system present the development of the efforts undertaken. A common and the most important element of all these strategies is the induction of a DNA double strand brake (DSB) in a preselected target loci. DSB repair induced in a living cell enables, depending on the experimental system, either the targeted mutagenesis or, in more advanced approaches, a precise replacement of the selected genomic region with an engineered fragment of DNA. The system based on CRISPR/Cas9 offers, comparing with other strategies, a relatively simple method of in vivo targeting of the preselected genomic locus. The induced mutations represent either SNP or Indels and they are indistinguishable from natural or conventionally induced mutations.

The process of a targeted induction of DSB relies on the activity of the nucleoprotein complex built of a Cas9 endonuclease and a single guided RNA (sgRNA). The complex can be delivered into the living cell through a direct transfer of the Cas9/sgRNA molecules to plant cells or by a genetic transformation with genes encoding Cas9 and sgRNA followed by their expression and in vivo production of Cas9/sgRNA. In either case the $23 \mathrm{nt}$ long sgRNA, which is complementary to the selected region of the target gene, associates with Cas9 protein and directs it to the target site of gDNA. Since the locus of sgRNA and Cas9 encoding genes and the locus of induced by the system mutation are not linked, it is possible to segregate both lociand to obtain mutated plants with no foreign DNA. This opens up the possibility to obtain mutated plants with novel traits, which might be considered as non-GMO.

The prerequisite of CRISPR/Cas9 application in plant biotechnology is a detailed knowledge of the target genes. In particular it is: 1) knowledge of the genomic and coding sequence of the gene, 2) information on the paralogues number of the gene, 3) information on the homologues of the gene in allopolyploids and 4) knowledge of the biological functions of the gene. The known gDNA sequence of the gene, its structure and function are the basis for the selection of the best region for targeted mutagenesis. There are numerous articles on CRISPR/Cas9 applications in plant sciences. So far the system has been successfully used in the most important crops such as rice, wheat, barley and soybean. In many cases the induced mutation led to novel useful traits and, what is also important, these plants contained no Cas9/sgRNA encoding genes. In countries where approval for commercialization depends on the characteristics of the plant itself (so called "product-based legislation") such plants do not fall into GMO-based legislation or the legislation is much simpler than for GM crops. In countries (including EU) where approval for commercialization considers not only the resultant plants but also its pedigree and the used technology (so called "process-based legislation") the way for commercialization is currently similar as for GM crops. Despite the fact that technology offers great advantages for plant biotechnology and plant breeding the benefits derived from its application will entirely go to the countries which will be open for its approval. This obvious imbalance stirs a discussion on the role of genome editing in green biotechnology. The current list of species, traits and cases already approved for commercialization as well as the discussion of the prospects of the technology in modern agriculture will be presented.

\section{Acknowledgements}

The work is financed by the National Science Centre, grant No. UMO-2015/17/D/NZ9/02020 (SG). 


\title{
Regulation of somatic embryogenesis induction in Medicago truncatula Gaertn. at the physiological and molecular level
}

\author{
EWA KĘPCZYŃSKA \\ Department of Plant Biotechnology, Faculty of Biology, University of Szczecin, Szczecin, Poland \\ * ekepcz@univ.szczecin.pl
}

In addition to in vitro somatic embryogenesis (SE) being a proven tool for massive propagation of commercial crops, it provides a good model system to study physiological, biochemical and molecular events during plant embryogenesis. It is a complex biological process in which somatic plant cells utilize their capacity of inducing an embryo and subsequently regenerating a complete plant. Competence to induce SE is known to be highly correlated with the genotype, as exemplified by Medicago truncatula cv. Jemalong embryogenic M9-10a (E) variant, a crop model for the study of SE. The line has been derived directly from non-embryogenic M9 (NE) genotype, which makes it possible to compare the states with "switched on" or "switched off" SE. In vitro SE in angiosperm and gymnosperm plants is regarded to be triggered primarily by auxins and cytokinins. However, our knowledge on the participation of other phytohormones such as gibberelins $\left(\mathrm{GA}_{\mathrm{s}}\right)$ and abscisic acid (ABA) is still far from complete.

To date, there have been no data concerning the analysis of simultaneous endogenous levels of gibberellin $\mathrm{A}_{3}$ $\left(\mathrm{GA}_{3}\right)$, ABA and IAA during the SE induction phase. As indicated by the LC-MS analysis of tissues of both $M$. truncatula lines sampled at five time-points (day 0,2, 7, 14 and 21) during the induction phase, the lines differ not only in the content of the primary explants phytohormones mentioned, but also in the dynamics of changes in the contents during the entire induction phase. The results obtained suggest that all the phytohormones mentioned participate in the SE induction in $M$. truncatula. Expression profiling confirmed the up-regulation of the newly identified genes encoding the enzymes involved in the biosynthesis and catabolism of gibberellins and ABA during the induction phase in $M$. truncatula. The presence of several genes encoding classical transcription factors during SE induction (WUS, WOX5, SERF, SERK1, SERK2) was confirmed. Moreover, the LEC1, L1L, LEC2, FUS3, ABI3, $S T M, A G L 15$ and $B B M$ genes were identified in the $M$. truncatula genome. All these genes, except $A G L 15$, can be suitable markers with which to monitor the progress of SE. Genes coding proteins from the Polycomb Repressive Complex1 (PRC1-LHP1, RING1a, BMI1a, EMF1, VRN1), Polycomb Repressive Complex2 (PRC2-CLF, SWN, FIE, MSI1, VRN2) and Tritorax Group (TrXG-ATX2, ATX3.1, ATX3.2 and ATX5-like), the critical epigenetic regulators of plant development, were identified in the $M$. truncatula genome for the first time, and their expression during SE induction was analyzed. The expression of the $P R C 1, P R C 2$ and $\operatorname{Tr} G G$ protein-coding genes in primary leaf explants was lower in the embryogenic than in the non-embrogenic line, which may suggest that primary explants of the two lines differ in their chromatin methylation level.

\section{Acknowledgements}

This work was supported by the National Science Centre (NCN) grant No. NN303801340. 


\title{
microRNA - small molecules with a huge impact on the regulation of developmental plasticity of plant somatic cells cultured in vitro
}

\author{
anna Maria WóJciK*, Maegorzata Danuta GaJ \\ Department of Genetics, Faculty of Biology and Environmental Protection, University of Silesia, Katowice, Poland \\ *anna.wojcik@us.edu.pl
}

microRNAs (miRNAs), the products of MIRNA genes, belong to the small non-coding RNAs and regulate gene expression by degradation of the targeted mRNAs or translational repression. The key role of miRNAs in the regulation of plant development in vivo has been widely documented and recently a regulatory impact of miRNAs on morphogenic processes induced in in vitro cultured tissue has become evident. The reports demonstrated the engagement of miRNAs in callus production, organogenesis and somatic embryogenesis (SE) induced in vitro in cultured explants of different plants.

Studies on SE in Arabidopsis, a model in plant genomics, have recently contributed significantly to our current knowledge on the genetic regulation of plant cell totipotency. Consistent with a common belief that the embryogenic transition of differentiated somatic cells requires extensive reprogramming of the cell transcriptome, an intensive modulation of the genes with regulatory function including those encoding transcription factors (TFs) and microRNAs (miRNAs) were indicated to control SE induction in Arabidopsis [1, 2].

Several $T F$ genes that play a decisive/key role in SE induction have been identified, including, BBM, WUS, AGL15 and LEC1, LEC2 while the role of miRNAs was assumed to be pivotal in the regulation of the SE-involved TFs, given that almost $80 \%$ of the miRNA-targeted genes encode TFs. And indeed, numerous miRNAs were reported to be expressed during the SE of different plants including Arabidopsis thaliana, Citrus sinensis, Dimocarpus longan, Gossypium hirsutum, Larix kaempferi, Larix leptolepis, Manihot esculenta, and Zea mays. Moreover, a mutation in the DCL1 gene that encodes the key enzyme of miRNA biosynthesis was found to inhibit the SE response in Arabidopsis. Although these studies confirmed the engagement of miRNAs in the embryogenic transition that is induced in vitro, our knowledge about the biological function of the specific miRNAs and the targeted genes in the SE induction is still limited.

Hence, we analyzed several miRNAs of differential expression in the embryogenic culture of Arabidopsis [2] from the functionality point of view. Given that auxin plays a key role in SE induction we focused our analysis on the miRNAs that are related to auxin. Thus we studied miR393, miR160, miR390, and miR165/166 that control in vivo the genes encoding auxin receptors, ARF transcription factors and TFs (PHB/PHV) controlling LEC2 that activates auxin biosynthesis in $\mathrm{SE}$, respectively. The analysis involving various genomic approaches provided experimental evidence on the regulatory role of miR393, miR160, miR390, miR165/166 and targeted genes during SE induction were indicated [2-4].

The role of auxin-related miR393, miR160, and miR165/166 in the induction of the embryogenic program triggered in the somatic cells of Arabidopsis cultured in vitro was demonstrated. The auxin-related miRNAs regulate the SE induction via the control of genes involved in the perception (TIR1, AFB2), signaling (ARF10, ARF16), and synthesis ( $L E C 2)$ of auxin. It has been proposed that the complex interplay involving regulatory interactions that act within and between the miR160- and miR165/166-mediated pathways need further experimental validation. Identification of other targets of the SE-involved miRNAs, and in particular those related to hormone- and stressresponses, is essential to fully define the genetic network controlling SE induction.

\section{References}

[1] Gliwicka et al. (2013) doi: 10.1371/journal.pone.0069261.

[2] Szyrajew et al. (2017) doi: 10.3389/fpls.2017.00018.

[3] Wójcik and Gaj (2016) doi: 10.1007/s00425-016-2505-7.

[4] Wójcik et al. (2017) doi: 10.3389/fpls.2017.02024.

This work was supported by a grant from the National Science Centre in Poland (PRELUDIUM 2016/21/N/NZ2/01718). 


\title{
Techniques of the gene function assessment using GMO and EMO methods
}

\author{
MAGDALENA ŻUK *, JAN SZOPA-SKÓRKOWSKI \\ Department of Genetic Biochemistry, Faculty of Biotechnology, University of Wrocław, Wrocław, Poland \\ *mzuk@ibmb.uni.wroc.pl
}

The development of DNA sequencing methods has resulted in a vast accumulation of the genomic data in the databases. Thus, the great challenge for biology is to determine the function of the identified sequences and to use them for biotechnology purposes. The most effective tool to realize this task is generation of genetically modified organisms. Until recently the GMO generating methodology has been based on the making of "mutants" by knocking out genes through transposon tagging, RNAi, etc. was the most frequently used. Very recently the extension of genes modification toolbox has been achieved owing to the possibility of introducing gene changes or new genes in a more targeted manner. Clustered, regularly interspaced short palindromic repeats systems (CRISPR), comprising Cas9 endonucleases and a single guide RNAs (gRNAs), and transcription activator-like effector nucleases (TALENs) have emerged as potent biotechnological tools for genes modification. The applying of both Cas $9 / \mathrm{sgRNA}$ and TALENs is precise genetic alterations within any genome as demonstrated in several plant examples. It should, however, be pointed out that both technologies are very efficient for the gene "loss of function" while gene replacement for the "gain of function" requires more research. Although the GMO generating methodology is the most frequently used, it is rather bothersome and time consuming. Another drawback that is frequently stressed is the fact that the genome continuity is physically broken when new, external or self-altered sequence is introduced. Besides, GMOs are seldom accepted outside of the laboratory, which strongly limits their application for wide industry.

It is thus a great challenge for the gene engineering methodology to develop a method which is fast, efficient, reliable and most importantly based on epigenetic approaches. The engineered CRISPR/Cas system to be nucleaseinactive may serve as a suitable DNA-targeting module for activation-induced cytidine deaminase (AID) to catalyze $\mathrm{C}$ to $\mathrm{T}$ changes which remind a natural process based on $\mathrm{C}$ residue methylation. AID-generated deoxyuridine in DNA is mutagenic as it can be miss-recognized as deoxythymine, resulting in $\mathrm{C}$ to $\mathrm{T}$ mutations. It was demonstrated in yeast that point mutations were dominantly induced at cytosines within the range of three to five bases surrounding the -18 position upstream of the PAM sequence on the non-complementary strand to gRNA. Thus, the nucleasedeficient CRISPR/Cas system may serve as a suitable DNA-targeting module for site-specific mutagenesis without breaking the DNA strands continuity.

Recently, several studies have proposed a targeted genome editing based on the plant treatment with short oligonucleotide sequences (OLIGO) that are homologues to the gene of interest. The technology was firstly applied for the modification of mammalian genes. In most cases OLIGO silences the target gene by interference but in a few cases the target gene activation has been reported.

Our recent data suggest that OLIGO technology is effective in the analysis of the gene function as well as in the generating new types of plants. The changes in the methylation of the endogenous $\beta$-1,3-glucanase ( $\beta$-glu), lycopen $\beta$-cyclase (LCB) and the chalcone synthase (CHS) genes were obtained upon treating flax with short oligodeoxynucleotides antisense to the coding region. In vitro cultured plants and F3 generation flax plants were characterized by up-regulation of $\beta$-glu, suppression of LCB and CHS genes, changes in the methylation of CCGG motif of the target genes and total DNA and, more importantly, plants obtained by this technology showed similar features as respective GMOs. This suggests that epigenetic tools might revolutionize the process of crop plants improvement. 


\title{
Genome editing in barley - its application in gene function studies and modifications of useful traits
}

\author{
Sebastian Gasparis *, Maciej Kata, Mateusz Przyborowski, Waclaw OrczyK, Anna Nadolska-Orczyk \\ Department of Functional Genomics, Plant Breeding and Acclimatization Institute - National Research Institute, Radzików, Poland \\ *s.gasparis@ihar.edu.pl
}

Genome editing technologies allow modification of virtually any genomic DNA sequence in a broad range of organisms. This technique relies on the application of sequence specific endonucleases and a cellular DNA repair system for precise modification of genome sequences. In practice, the endonuclease is fused with specific DNA binding domains and transferred to the nucleus where it generates double strand DNA breaks (DSB) in a site specific manner. As a result of errors in the DSB repair process, small insertions, deletions or rearrangements may occur at the joining site of broken DNA leading to gene knock-out mutations. In more advanced applications, the DSB can be generated to introduce new DNA sequence at a specific genomic location. This process may be utilized for a precise molecular design of crop cultivars or for functional/structural analysis of sequenced genes. The clustered regularly interspaced short palindromic repeat Cas9-associated (CRISPR/Cas9) system is recently developed, most innovative genome editing technology. It was adapted from the prokaryotic adaptive immunity system against invading foreign DNA. This system consists of two elements: a Cas9 endonuclease protein and a single, non-coding RNA molecule (single guided RNA, sgRNA) that guides the Cas9 nuclease to the target DNA sequence. Depending on the application, the CRISPR/Cas9 elements can be introduced into the host cells in two fashions: 1) by stable genetic transformation as a single DNA construct or, 2) by transient assay as a nucleoprotein complex.

With its completely sequenced genome, barley may be considered as a diploid model species for closely related hexaploid cereals and the application of a CRISPR/Cas9 technology gives a great opportunity to study gene functions in cereals.

In this study we developed an optimized CRSIPR/Cas9 system for efficient genome editing in barley. The optimization steps included the design of synthetic, codon optimized Cas9 gene and the sgRNA constructs with enhanced expression in monocot plants. Additionally, a polycistronic tRNA-gRNA (PTG) construct was designed for simultaneous editing of multiple target genes. A set of binary vectors for both simplex and multiplex editing of selected barley genes was prepared. The CRSIPR/Cas9 vectors were used to produce transgenic barley plants by Agrobacterium-mediated transformation. The effectiveness of the CRISPR/Cas9 system was demonstrated by generating targeted mutations in the ethylene response transcription factor gene $\mathrm{Nud}$. The obtained mutant plants possessed a new visible trait, i.e. naked (hulles) grains.

Another set of CRSIPR/Cas9 vectors were used for targeted mutagenesis of two barley $C K X$ genes, $H_{v} C K X 1$ and $H_{v} C K X 3$ belonging to small family of genes encoding cytokinin oxidase/dehydrogenase enzymes that regulate endogenous levels of cytokinin hormones. The $C K X$ genes show different developmental and tissue specific expression patterns suggesting their specialized functions in certain organs.

$H_{v} C K X 1$ and $H_{v} C K X 3$ were edited separately in two independent experiments using two different sgRNAs specific for each gene. In the third experiment, a single PTG construct was used for simultaneous editing of both $C K X$ genes. The rates of mutations generated in $\mathrm{T}_{0}$ plants ranged from 18 to $88 \%$. In most of the tested transgenic lines the induced mutation were transmitted to the $\mathrm{T}_{1}$ generation which allowed for selection of homozygous $c k x 1$ or ckx3 mutants. The first results showed significantly decreased activity of CKX enzymes in developing spikes of $c k x 1$ mutants. Further studies will be performed to assess the CKX enzymes activity in other organs and their impact on cytokinin metabolism.

\section{Acknowledgements}

This research is supported by the National Science Center grant UMO-2015/17/D/NZ9/02020. 


\title{
Biolistic transformation of Miscanthus sinensis and Miscanthus $\times$ giganteus
}

\author{
Karolina Sobańska, Joanna Cerazy-Waliszewska, Aurelia Ślusarkiewicz-Jarzina, \\ Aleksandra Ponitka, StanisŁaw Jeżowski, Tomasz PNiewski * \\ Institute of Plant Genetics, Polish Academy of Sciences, Poznań, Poland \\ *tpn@igr.poznan.pl
}

Miscanthus is a genus of about 20 species of perennial giant grasses which have great potential of up to $30 \mathrm{t} / \mathrm{ha}$ of biomass yield in countries of moderate climate. This biomass can be used as a renewable feedstock for conversion into bioenergy or biofuels such as bioethanol. Biotechnological methods based on genetic engineering and plant transformation can be an essential complement of the efforts to improve the miscanthus biomass yield or its quality.

Here we report the establishment of a transformation system of Miscanthus sinensis and M. x giganteus through microparticle bombardment (a biolistic method).

Preliminary tests showed that hygromycin B is an appropriate selection factor for the miscanthus species. Therefore two plasmid vectors containing $h p t$ marker gene (coding hygromycin phosphotransferase) were used in the studies: pCAMBIA1201 and optimised pCAHGA. The vector pCAHGA was derived from pCAMBIA1201 and comprised the coding sequence of $o h p t$ gene, i.e. an optimised according to the codon usage in grasses and placed under control of the ubiquitin1 gene promoter (UBQ) from Zea mays (GenBank J× 947345.1), and the GUS (uid) coding sequence driven by the promoter of actin gene - actin1 (Act1) from Oryza sativa (GenBank S44221.1). In the studies, three genotypes of $M$. sinensis (Ms1, Ms16 and Ms17) and two genotypes of $M . \times$ giganteus (MG3 and MG4) were transformed with plasmid DNA coated on gold microcarriers. Several combinations of microbombardment parameters as pressure and distance to target were tested. About 8-10-week-old embryogenic calli ( 80 per variant) obtained from immature inflorescences were used as plant material for transformation. The callus induction was conducted on the C-17 as a basal medium, supplemented with 2.4-D $\left(5.0 \mathrm{mg} / \mathrm{dm}^{3}\right), \mathrm{BAP}\left(0.5 \mathrm{mg} / \mathrm{dm}^{3}\right)$ and $90 \mathrm{~g} / \mathrm{dm}^{3}$ maltose. The callus was transferred on the osmotic medium (the same callus induction medium additionally supplemented with $36.4 \mathrm{~g} / \mathrm{dm}^{3}$ sorbitol and mannitol each) 24 hours before transformation. All steps of plant regeneration, including plantlet development, growth and tillering and rooting, were conducted on 190-2 medium supplemented with KIN $\left(0.5 \mathrm{mg} / \mathrm{dm}^{3}\right)$ and NAA $\left(0.5 \mathrm{mg} / \mathrm{dm}^{3}\right)$ and $5.0 \mathrm{mg} / \mathrm{dm}^{3}$ of hygromycin as a selection agent. Finally, 35 transgenic plants, 3 for $M$. sinensis and 32 for $M$. $\times$ giganteus, were obtained and only for pCAHGA vector. These were micropropagated up to 5 clones, which were rooted and transferred to soil. Clones were analysed using PCR to detect transgenes, both ohpt and uid. T-DNA genomic integration was confirmed in all clones of all 35 transformants. The transformation efficiency varied from 3.8 to $11.3 \%$ and the mean was $6.3 \%$. The transgenic character of the obtained plants was also confirmed by RT-PCR and fluorometric GUS assay. Further analyses of the obtained transgenic plants, such as the determination of the number of transgene copies and the transcription activity of the ohpt gene using ddPCR, as well as the HPT activity assay, are in progress. This is the first report on the establishment of the biolistic transformation of $M . \times$ giganteus and the essential progress in the transformation of $M$. sinensis.

This research was realised as a part of SORMISOL project, No. PBS1/A8/9/2012, supported by the NCRD. 


\title{
Evaluation of the usefulness of ISSR markers in the research of Chrysanthemum $\times$ grandiflorum Ramat./Kitam. plants obtained via somatic embryogenesis
}

\author{
Emilia MichatowsKa, Justyna Lema-RUMińsKa* \\ UTP University of Science and Technology in Bydgoszcz, Bydgoszcz, Poland \\ *lem-rum@utp.edu.pl
}

Chrysanthemum (Chrysanthemum $\times$ grandiflorum Ramat./Kitam.) occupies a high position in the gardening market, and its production continues to increase every year. To satisfy the growing needs of the market, new methods of propagation and breeding are being sought. One of the most efficient regenerative methods is somatic embryogenesis (SE), which can be used both for micropropagation of the plants and for breeding of new cultivars at the stage of regeneration from singular somatic cells. The greatest SE advantage is its efficiency, specifically a high number of somatic embryos regenerated from a single explant. An underrated but very important aspect of somatic embryogenesis is a possible variation in plants regenerated applying that method, often resulting from adding high concentrations of growth regulators to the medium at the ES induction stage, and it is related to the callus phase which frequently mediates the regeneration of somatic embryos. The variation can be applied in new cultivars breeding programmes. To detect the variation at the genetic level, the molecular markers are used. Inter Simple Sequence Repeat primers (ISSR) based on the amplification of DNA segments are placed between two opposite-directed repeat regions which comprise microsatellite repeat sequences. These repetitions are characterized by a high variation and they are widespread throughout the genome.

The objective of the study was to determine the usefulness of six different ISSR primers for the genetic stability research in the chrysanthemum plants obtained through the somatic embryogenesis. Experimental material consisted of the lines of two cultivars: "Lady Salmon" (LS1-LS5) and "Lady Vitroflora" (LV1-LV5). The plants obtained from meristems constituted the controls. DNA was isolated from the chrysanthemum leaves with the use of Genomic Mini AX Plant kit (A\&A Biotechnology firm). The electrophoretic separation was performed on $1.5 \%$ agarose gel with the voltage current equaling $90 \mathrm{~V}$ for 20 minutes and $110 \mathrm{~V}$ for the next 90 minutes. The analysis of the reaction products was made using GelAnalyzer 2010 program. The primers used in the PCR reaction showed a notable polymorphism between the genotypes analyzed. In "Lady Salmon" cultivars 236 products were obtained; 23 bands were polymorphic, while in "Lady Vitroflora" 221 products were produced, from which 19 showed a variability. All the primers (S1-S6) showed a polymorphism in the inter-microsatellite region; the highest polymorphism in "Lady Salmon" and "Lady Vitroflora" lines with primer S3, whereas the lowest - in "Lady Salmon" cultivar with primers S5 and S6, and in "Lady Vitroflora" cultivar with primer S1. The highest average polymorphism was observed with the use of primer S2 and the lowest - with primer S5, which allowed to select the primers for genetic variation analyses. ISSR markers find their application in the assessment of the genetic stability of Chrysanthemum $\times$ grandiflorum Ramat./Kitam. obtained as a result of the somatic embryogenesis. 


\title{
Silver and copper nanoparticles in in vitro culture of chrysanthemum: disinfecting properties and influence on adventitious shoot regeneration
}

\author{
AlicJa TyMOSZUK \\ Faculty of Agriculture and Biotechnology, UTP University of Science and Technology in Bydgoszcz, \\ Bydgoszcz, Poland \\ *alicja.tymoszuk@utp.edu.pl
}

In the recent years an intensive development of nanotechnology has been observed. Nanoparticles are characterized by a high chemical reactivity and easy interaction with biological material. Disinfecting properties of metal nanoparticles can be very useful for effective reduction of microbiological contamination during in vitro culture initiation. Their influence on plant growth, development and genetic stability is also noteworthy.

In the present study the usefulness of silver, gold and copper nanoparticles in disinfection of Chrysanthemum $\times$ grandiflorum/Ramat./ Kitam. "Richmond" shoot tips was evaluated. The explants were first rinsed under running water, incubated in $5 \%$ detergent solution for $5 \mathrm{~min}$, and then in $70 \%$ ethanol solution for $5 \mathrm{~s}$. Next, the explants were incubated in nanocolloids (Ag; $\mathrm{Au}$; $\mathrm{Cu}$ or $\mathrm{Ag}$ with $\mathrm{Cu}, 5$ or $10 \mathrm{ppm}$, for 6 or $9 \mathrm{~min}$ ). Then they were rinsed for $5 \mathrm{~min}$ in sterile distilled water. The explants were dried on sterile paper and inoculated, in polar position, on the standard MS (Murashige and Skoog, 1962) medium. During four successive weeks, observations were made for the appearance of contamination. The share of disinfected cultures, depending on the research object, ranged from 50 to $100 \%$. The most effective were: gold, silver and silver mixed with copper; $93.75 \%, 87.50 \%$ and $87.50 \%$ contaminationfree cultures, respectively. The share of disinfected cultures for copper nanoparticles was 59.38\%. The nanocolloids demonstrated a satisfying antibacterial and antifungal activity even at low concentrations and a short time of disinfection.

In the second study, internodes of Chrysanthemum × grandiflorum "Lilac Wonder", "Richmond" and "Satinbleu" were inoculated horizontally onto the MS medium supplemented with $0.6 \mathrm{mg} \cdot \mathrm{dm}^{-3}$ benzylaminopurine (BAP) and $2 \mathrm{mg} \cdot \mathrm{dm}^{-3}$ indoleacetic acid (IAA) for adventitious shoots regeneration. Silver and copper nanocolloids, $50 \mathrm{~nm}$ in size, were also added to the medium at the concentrationof of 5,10 or $18.5 \mathrm{ppm}$. The regeneration was conducted for 10 successive weeks. The obtained results showed that the addition of silver and copper nanoparticles to the medium significantly reduced the efficiency of adventitious shoots regeneration, particularly at the concentrations of 10 and $18.5 \mathrm{ppm}$.

The effect of silver nanocolloid (18.5 ppm) added to the medium on the genetic stability of chrysanthemum was determined. Genomic DNA was isolated from leaves of regenerated adventitious shoots and from control plants propagated in vitro using single-node method on the MS medium without plant growth regulators and nanoparticles. The genetic stability was determined by RADP-PCR and ISSR-PCR method. In each experiment, five primers were tested. Then the electrophoretic separation of PCR products on an agarose gel was performed. Based on the analysis of band patterns, it was found that adventitious shoots regenerated on a medium with a silver nanocolloid demonstrated genetic variation as compared to the control plants. This was also confirmed by the phenotypic evaluation of flowering plants. Nanosilver particles can be a potential source of variability in chrysanthemum breeding. 


\title{
Directed and hereditary modification in CHS gene methylation induced by oligonucleotides
}

\author{
MAGDAlena DZIALO $^{1 *}$, JAN SzOPA-SKóRKOWSKI ${ }^{2}$, MAGDALENa ŻUK ${ }^{1}$ \\ ${ }^{1}$ Department of Genetic Biochemistry, Faculty of Biotechnology, University of Wroclaw, Wrocław, Poland \\ ${ }^{2}$ Department of Genetics, Plant Breeding and Seed Production, Wroclaw University of Environmental and Life Sciences, Wrocław, Poland \\ *magdalena.dzialo@uwr.edu.pl
}

Chalcone synthase (CHS) has been recognized as a crucial enzyme in the phenylpropanoid biosynthesis pathway. Apart from its leading role in the production of phenolic compounds (primarily flavonoids) with many valuable biological activities beneficial to medicine, $\mathrm{CHS}$ is also well appreciated in the science. Genetic engineering definitely facilitates expanding the knowledge of the function and genetics of CHS in plants. The CHS gene is one of the most intensively studied genes in flax. The engineering of the CHS gene through genetic approaches has already been widely investigated. Considering the numerous restrictions concerning the application of the genetically modified (GM) crops, the main purpose of this research was the optimization of the plant's modulation via epigenetics. For the last two decades, epigenetics has been considered as an equally important source of organism variability as genetic modifications. Epigenetic changes refer to the chromatin modifications that do not occur due to the interruption of the nucleic acid sequence, such as DNA methylation or various histone modifications. In our study the plants modified through two methods were compared: a widely popular agrotransformation and a relatively novel oligodeoxynucleotide (OLIGO) strategy. It was recently highlighted that the OLIGOs technique can be used in a quick analysis of the gene function before proceeding to a vector-mediated transformation. In order to understand the molecular background of the epigenetic variation in more detail and evaluate the use of OLIGOs as a tool for predictable and stable gene engineering, we concentrated on the integration of gene expression and gene-body methylation. The treatment of flax with a series of short oligonucleotides homologous to a different part of CHS gene isoforms revealed that those directed to the regulatory gene regions activated gene expression, while those directed to non-coding region caused a reduction of the gene activity, and those homologous to a coding region may have a variable influence on its activity. Also the methylation status of plants upon OLIGO treatment was changed and was associated with gene expression changes. However, only certain - CCGG - motifs along gene sequence were affected. The changes concerned the same - CCGG - gene motifs and resulted in the modification of the gene activity. It thus suggests the epigenetic-genetic interaction in the gene expression regulation mediated by DNA methylation. The analyzed DNA motifs of CHS flax gene were more accessible for methylation when located within $\mathrm{CpG}$ island. The motifs methylation led also to the rearrangement of the nucleosome location and thus to the changes in the gene expression. However, another mechanism such as the competition for transcription factors between OLIGO and its gene homolog cannot be excluded. The obtained results suggest that the OLIGO action is highly specific and leads to the induction of a heritable genome diversification. Consequently, an oligodeoxynucleotides technology might be a valuable alternative for the improvement of crops. 


\title{
Induced rye embryogenesis - a summary of 35 years of research
}

\author{
JANUSZ ZIMNY \\ Plant Breeding and Acclimatization Institute - National Research Institute, Radzików, Poland \\ *j.zimny@ihar.edu.pl
}

The paper presents the progress made over the past 35 years in the field of rye plant regeneration in in vitro cultures at the Polish Academy of Sciences Botanical Garden - Center for Biological Diversity Conservation in Powsin (PAS BG-CBDC) and at the Plant Breeding and Acclimatization Institute Radzików (PBAI). In the 1970s, a number of studies were carried out in Poland on the regeneration of plants in rye in in vitro cultures. They were focused mainly on regeneration from microspores, although it was already obvious that for the plant material used for regeneration to be morphogenic, it should contain a meristatically active tissue with a strong cell division potential. For this reason, in the case of rye, young inflorescences, apical and root meristems, or leaf base were used. Jan Rybczyński in the Institute of Plant Genetics PAS in Poznań, concentrated his studies on the immature embryo as an explant for cultures and dissected elements of the embryo as a source of callus, capable of regenerating plants. Subsequent work carried out at the PAS BG-CBDC in Powsin showed the ability of many genotypes of Secale cereale and some wild species of the taxa to induce somatic rye embryos. Further research on somatic embryogenesis was continued at the Max Planck Institute in Cologne and led to the publication of an efficient regeneration method. In this paper the process has also been described morphologically and histologically. At the same time, methods of isolation and culture of rye protoplasts and their transformation using PEG were established. The developed method of somatic embryogenesis was used in the 1990s in order to efficiently transform triticale and rye at the PBAI in cooperation with the University of Hamburg. After 2000, in cooperation with Dr. Lucjan Madej, at PBAI, efforts were made to regenerate haploid plants from rye microspores. The application of selected stress chaning the development path of microspores into the sporophytic one and the work on the selection of androgenic genotypes led to the regeneration of doubled haploid rye with extremely high efficiency. In our research, we investigated the effect of various stresses on microspore viability. We used also nine different stress combinations to induce androgenesis from anthers. The experiments were carried out on microspores and anthers of winter rye breeding lines. The results showed a correlation between the genotype and the stress used, and the level of androgenesis induction and microspore mortality, at an early stage of cultivation. The survival rate of microspores was the highest for two genotypes, after pre-cooling at $4{ }^{\circ} \mathrm{C}$ for two weeks, and further pre-cultivation of anther in a mannitol solution at $4{ }^{\circ} \mathrm{C}$ for seven days. The cooling of shoots for three weeks was equally effective. The use of heat shock $\left(32^{\circ} \mathrm{C}\right)$ led to $100 \%$ cell death. We are presenting the first so highly efficient division of isolated rye microspores and regeneration of almost 2 thousand green plants of rye from anthers.

After many years of research we can report high a regeneration efficiency of rye that enables to use it for testing gene expression in transformed plants and to shorten the breeding cycle of new hybrid cultivars. 


\title{
Activity of an antioxidative system as a factor determining microspore embryogenesis in triticale $(\times$ Triticosecale Wittm.)
}

\author{
IWONA ŻUR \\ The Franciszek Górski Institute of Plant Physiology, Polish Academy of Sciences, Kraków, Poland \\ *i.zur@ifr-pan.edu.pl
}

Isolated and in vitro cultured microspores are particularly interesting study objects due to their potential to initiate an alternative, sporophytic pathway of development called microspore embryogenesis (ME), highly homological to zygotic embryo development in planta. A huge advantage of the in vitro culture method is the unique opportunity to microscopically visualize the process and to analyse molecular and physiological factors controlling and regulating its course on a single cell level. Moreover, its final result - haploid/doubled haploid (DH) plants are highly advantageous in many domains of basic studies, biotechnology and breeding. However, despite many efforts, an efficient ME induction required for its commercial application has only been developed in a limited number of plant species. The main cause of the very slow progress in a wider application of DH technology is the fact that ME is controlled by a great number of endogenous and environmental factors constituting a network of dynamic interactions.

One of the most important requirements for an effective $\mathrm{ME}$ is the proper selection of the stress used as a trigger inducing microspore reprogramming. Stress treatment has to be strong enough to start a signal for the redirection of microspore development in a large number of cells, but not exceed the level of cell stress tolerance. As very different stress factors can be used for $\mathrm{ME}$ initiation, microspore reprogramming has to be triggered by one of general stress reactions. A possible candidate is the generation of reactive oxygen species (ROS) inevitably associated with aerobic cell metabolism but significantly enhanced in stress conditions. An excessive production of ROS causes a progressive oxidative damage and can ultimately result in cell death. On the other hand, ROS have been recognized as signalling molecules involved in the regulation of a variety of cellular processes, including the increase of tolerance to various environmental stresses. The effect of the stress depends on the precise equilibrium between ROS generation and the activity of the antioxidative system, composed of enzymatic and low molecular weight antioxidants present in plant cells.

To verify this hypothesis, the antioxidative system activity under various stress treatments and its association with ME effectiveness were studied in anther and isolated microspore cultures of triticale ( $\times$ Triticosecale Wittm.) with the use of several DH lines significantly varied in respect of their embryogenic potential. The results confirm that a properly balanced activity of the antioxidative system seems to be the first, though not the only one, prerequisite for efficient $\mathrm{ME}$ initiation.

The research was performed in the frame of projects NN310452638 and 2015/18/M/NZ3/00348 financed by the National Science Centre and supported by institutional funding of the Institute of Plant Physiology Polish Academy of Sciences, project No. T1Zb2/2016. 


\title{
Production of oat (Avena sativa L.) doubled haploids via androgenesis and wide crosses
}

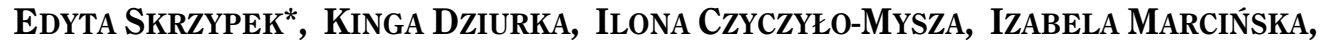 \\ Kamila KaploniaK, Angelika Noga, Marzena Warcho€ \\ The Franciszek Górski Institute of Plant Physiology, Polish Academy of Sciences, Kraków, Poland \\ * e.skrzypek@ifr-pan.edu.pl
}

Oat (Avena sativa L.) is gaining importance due to the health-promoting properties, e.g. as an ingredient of food, medicinal and cosmetic products. New cultivars that are created are mainly based on a few years of inbred crosses to get plants with high homozygosity and subsequent selections of individuals with desirable traits. The method allowing to shorten the above-mentioned procedure is to obtain doubled haploids (DH). Oat DH are produced by androgenesis and wide crosses. The most common problem in the oat anther culture is the very low percentage of obtained embryo-like structures (ca. $2 \%$ ) and regenerated plants (less than $0.01 \%$ ), in which albinism often occurs. Pollination of oat by maize seems to be more promising as the frequency of embryo formation is higher than in the anther cultures (to 9 and 3\%, respectively). The efficiency of the wide crossing method depends on the donor plants growing conditions and in vitro culture conditions, mainly temperature, light intensity and growth regulators in the regeneration media [1, 3, 4]. Formation of haploid embryos is also highly influenced by the genotype. After wide crossing, it is often possible to generate unstable chromosomal systems, as a result of which the male chromosomes are eliminated from the developing embryo during subsequent cell divisions in the early stages of embryogenesis. This leads to the development of highly homozygous plants with the desired genotype. After oat wide crossing, maize chromosomes or their fragments might be incorporated into the oat genome. Even half of the plants obtained by oat and maize crosses can be hybrids. Nearly $60 \%$ of hybrids are stable and fertile, but they frequently show a strong differential phenotypic effect [2]. Various morphological changes in the $\mathrm{F}_{1}$ progeny are observed depending on the maize chromosome added into the oat genome.

\section{Acknowledgements}

The research was funded by The National Centre for Research and Development, PBS3/B8/17/2015 and Institute of Plant Physiology PAS.

\section{References}

[1] Noga A., Skrzypek E., Warchoł M. et al. (2016) In Vitro Cell Dev. Biol. Plant. 52: 590-597.

[2] Rines H.W., Phillips R.L., Kynast R.G. et al. (2009) Theor. Appl. Genet. 119: 1255-1264.

[3] Skrzypek E., Warchoł M., Czyczyło-Mysza I. et al. (2016) Cereal Res. Commun. 44: 490-500.

[4] Warchoł M., Skrzypek E., Nowakowska A. et al. (2016) Plant Growth Regul. 78: 155-165. 


\title{
Meiotic restitution as a process determining doubling the chromosome number in haploids of triticale
}

\author{
SYLWIA OLESZCZUK \\ Department of Plant Biotechnology and Cytogenetics, \\ Plant Breeding and Acclimatization Institute- National Research Institute, Radzików, Poland \\ *s.oleszczuk@ihar.edu.pl
}

Poliploidization, plays a crucial role in plant speciation and evolution. Many studies confirmed the occurrence of one or more rounds of independent genome duplication events in most angiosperms [2]. In allopolyploid plants, such as triticale, an additional amount of genetic material allows it to significantly increase its resistance to disease, helps in the adaptation to the changing environment conditions and has a wide influence on the improvement of the quality of value in use. In such interspecific hybrids, poliploidization includes wide hybridization followed by a spontaneous doubling of the number of chromosomes. Among natural processes, a meiotic restitution is known as a cause of chromosome doubling and hence the fertility in several new hybrids [1]. In restitution, meiotic division of the cells is changed into a mitosis-like non-reductional process (sister chromatids are separated at the first meiotic division there is no the second meiotic division), forming dyads instead of tetrads at the end of meiosis II. This results in the formation of functional, unreduced gametes with the somatic chromosome number (2n) [4]. The participation in fertilization of such gametes leads to an increase of ploidy in the offspring compared with the parent. However, the molecular mechanism controlling the disruption of meiosis and leading to the production of unreduced gametes remains unknown despite of many analyses carried out at molecular and cytological levels.

Despite the presence of restitution in nature, only sporadically was it considered a phenomenon which determines the doubling of chromosomes number in haploid regenerants obtained via androgenesis. In androgenicgenerated plants, mainly the fusion of haploid nuclei at the early stages of the microspore divisions during in vitro culture as well as endoreduplication and c-mitosis were found to be responsible for genome doubling. Due to the fact that wheat, as one of the parent components of triticale, probably appeared as a result of meiotic restitution, it should be assumed that the process could also be present in triticale and be responsible for the creation of unreduced gametes. As a result of meiotic restitution, the plant forming from haploid cells are able to produce seeds without the necessity of applying mitotic inhibitors like colchicine. The discovery of genetic mechanisms responsible for the restitution may open interesting possibilities to translate this knowledge into its application in the generation of doubled haploid lines in plant breeding and in scientific research.

\section{References}

[1] Cai X., Xu S.S. (2007) Genome 8: 151-161.

[2] Jiao Y., Wickett N.J., Ayyampalayam S. et al. (2011) Nature 473: 97-100.

[3] Ramanna M.S., Jacobsen E. (2003) Euphytica 133: 3-18. 


\title{
Plant sex chromosomes in vivo and in vitro
}

\author{
ANDRZEJ JOACHIMIAK \\ Department of Plant Cytology and Embryology, Jagiellonian University, Kraków, Poland \\ *a.joachimiak@uj.edu.pl
}

Spatial separation of reproductive organs in plants is one of the mechanisms protecting them against self-fertilization and in Angiospermae it can be realized through monoecism or dioecism. Although dioecism is the best mechanism promoting cross-fertilization, it occurs in only 6\% of Angiospermae (14620 species out of 240000), belonging to the $7 \%$ of the genera and $38 \%$ of the families. The vast majority of dioecious plant species do not possess sex chromosomes, and consequently lacks chromosomal sex determination, which is common in animals. In about 30 species of Angiospermae the occurrence of heteromorphic sex chromosomes was shown and although it is a unique phenomenon in plants, it attracts attention of many researchers, especially geneticists and evolutionists. The dispersion within the genealogical tree proves that sex chromosomes in Angiospermae originated repeatedly and independently on one another and that they are evolutionarily young. Despite their young evolutionary age, both the systems of sex chromosomes and the systems of chromosomal sex determination in plants show significant differentiation.

Studying two genera of species possessing sex chromosomes and those not possessing them (Silene and Rumex) provided much interesting information about the origin and structure of sex chromosomes, the differentiation of the sex chromosome systems and sex determination. The basic established facts are as follows:

1) Sex chromosomes resulted from a pair of autosomes possessing two key genes (regions): one of them determined the formation of male organs (stamens), the other - female organs (pistils). Mutation inactivating the former resulted in the formation of chromosome $\mathrm{X}$ (proto-X), while mutation inactivating the latter - chromosome $\mathrm{Y}$ (proto-Y);

2) The primitive pair of sex chromosomes was homomorphic and its differentiation proceeded slowly. This process was triggered by a gradual loss of recombination between proto-X and proto-Y, which was necessary for maintaining dioecism (otherwise a decrease in fertility and probable recurrence of bisexuality would have occurred);

3) A loss of recombination resulted in changes occurring independently in both chromosomes, particularly, however, in chromosome $Y$, where degeneration of part of genes and accumulation of non-coding sequences occurred. One of the main mechanisms responsible for these phenomena was so called Muller's ratchet;

4) In plants, the evolution did not lead to a significant genetic degeneration of chromosome $Y$ or a decrease of its size (a common phenomenon in animals). Conversely, in plants chromosome $Y$ became bigger than chromosome $\mathrm{X}$. It suggests that differentiation of sex chromosomes in plants is subject to slightly different rules than in animals. However, these rules have not been sufficiently examined so far.

5) The primary system of sex chromosomes is a simple system $X X / X Y$, where the male sex is heterogametic. Secondarily, as a result of chromosomal mutations, complex systems (e.g., XX/XY1Y2 or X1X1X2X2/X1Y1X2Y2) could have appeared. The system of heteromorphic sex chromosomes based on female heterogamety (animal equivalent: $Z Z / Z W)$ in plants did not develop at all.

The majority of previous research on plant sex chromosomes was conducted on material (root tips, leaves, flowers) obtained directly from plants. Only in some cases in vitro cultures were used, especially in order to obtain root meristems (hairy roots cultures) easily. It is definitely not enough because in vitro experiments can be extremely helpful to explain such matters as degeneration of chromosome Y, epigenetic modifications of sex chromatin, differences between sexes at the biochemical and physiological levels as well as the cell response to different factors and stimuli. In the future this kind of research will definitely be conducted much more intensively. 


\title{
Oat (Avena sativa L.) anther culture: the effect of pretreatment and media composition on the embryo-like structures production
}

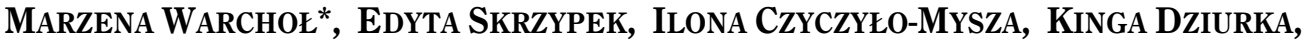 \\ IZABEla MarcińsKa, ANGElika Noga, KaMila KaPŁoniaK
}

Department of Biotechnology, The Franciszek Górski Institute of Plant Physiology, Polish Academy of Sciences, Kraków, Poland

*m.warchol@ifr-pan.edu.pl

Androgenesis is defined as a developmental pathway consisting in the change of the normal gametophytic development of microspores into a sporophytic one. Although androgenesis-based methods have been in recent years particularly successful in the many Gramineae, oat (Avena sativa L.) is still considered one of the most recalcitrant cereal crop with respect to doubled haploidy. Many endogenous and exogenous factors affect the embryogenic response of anthers in a culture, the most important of them being: the genotype, the stage of microspores development, pretreatment of anthers, the composition of the in vitro culture medium, and their interactions. Experiments were conducted to evaluate the factors influencing androgenesis, i.e. pretreatment and the composition of the induction media. Cultivars of oat (Avena sativa L.), Bingo and Chwat derived from Strzelce Plant Breeding Ltd. were used as sources of anthers. Donor plants were grown under controlled conditions, at $21 / 17^{\circ} \mathrm{C}$ day/night, $16 \mathrm{~h}$ photoperiod, in a greenhouse. The shoots were placed in Hoagland [1] liquid medium and cooled at $4^{\circ} \mathrm{C}$ for 2 or 3 weeks, and then half of them were placed at $32^{\circ} \mathrm{C}$ for 24 hours. Anthers were aseptically isolated on W14 media [4] and $\mathrm{C} 17$ [3] with the addition of auxin: 2,4-dichlorophenoxyacetic acid (2,4-D) in concentrations of 2.0 or $5.0 \mathrm{mg} / \mathrm{dm}^{3}$, picloram $0.5 \mathrm{mg} / \mathrm{dm}^{3}$, dicamba $0.5 \mathrm{mg} / \mathrm{dm}^{3}$ and naphthyl-1-acetic acid (NAA) $2.0 \mathrm{mg} / \mathrm{dm}^{3}$ and cytokinins: kinetin $0.5 \mathrm{mg} / \mathrm{dm}^{3}$, 6-benzylaminopurine (BAP) $0.5 \mathrm{mg} / \mathrm{dm}^{3}$. The medium was enriched with $9 \%$ maltose, solidified with $0.6 \%$ agar, and the $\mathrm{pH}$ was adjusted to 6.0 . The efficiency of the androgenesis was determined by the number of embryogenic structures (ELS) obtained from 100 anthers.

In the present research cold pre-treatment of oat panicles had a significant effect on the number of ELS which were formed on the all tested media. The most ELS were obtained when the anther was cultured on W14 medium with the addition of: 1) $5.0 \mathrm{mg} / \mathrm{dm}^{3}$ 2,4-D, $0.5 \mathrm{mg} / \mathrm{dm}^{3}$ kinetin and $2.0 \mathrm{mg} / \mathrm{dm}^{3} \mathrm{NAA}$ and 2) $2.0 \mathrm{mg} / \mathrm{dm}^{3} 2$,4-D and $0.5 \mathrm{mg} / \mathrm{dm}^{3}$ kinetin (1.6 ELS/100 anthers and $1.4 \mathrm{ELS} / 100$ anthers respectively). The number of ELS varied also between cultivars. In the cv. Chwat, 77 ELS were obtained (1.1 ELS /100 anthers), whereas in the cv. Bingo 29 ELS (0.5 ELS / 100 anthers).

\section{Acknowledgements}

The research was funded by The Franciszek Górski Institute of Plant Physiology, Polish Academy of Sciences.

\section{References}

[1] Hoagland D.R., Arnon D.I. (1938) California Agri. Exp. Station Circul. 347: 1-39.

[2] Murashige T., Skoog F. (1962) Physiol. Plant. 15: 473-497.

[3] Wang X.Z., Hu H. (1984) Plant Sci. Lett. 36: 237-239.

[4] Ouyang J.W., Jia S.E., Zhang C., Chen X.D., Feng G.H. (1989) Ann. Rep. Inst. Genet. Acad. Sin. (1986-1988): 91-92. 


\title{
Chloroplast differentiation during regeneration of green and albino plantlets in the microspore culture of barley
}

\author{
Monika Gajecka *, Marek Marzec, Beata Chmielewska, Janusz JeloneK, \\ JUSTYNA ZBIESZCZYK, IWONA SZAREJKo \\ Department of Genetics, University of Silesia, Katowice, Poland \\ *monika.gajecka@us.edu.pl
}

The application of doubled haploids (DHs) methods in breeding programs shortens the time required for the development of new varieties. Androgenesis is potentially the most effective method of DH production, but despite the optimised protocols for microspore embryogenesis in many cereal species, the effectiveness of androgenesis is vastly limited by frequent occurrence of albino plantlets among regenerants. For the induction of an embryogenic development of microspores in vitro, the application of stress, e.g. high or low temperature, or sugar and nitrogen starvation, is required to change the developmental pathway from the gametophytic to the sporophytic program. Microspores contain plastids, which give rise to chloroplasts in regenerating androgenic plantlets. During the in vivo pollen maturation, a vegetative cell contains proplastids which differentiate into amyloplasts to serve as starch storage for further pollen action. Due to the maternal inheritance, plastids enclosed in the generative cell are excluded or degraded. However, not all proplastids during in vitro culture differentiate into chloroplasts, which causes the regeneration of albino plantlets. This phenomenon reduces the use of some cultivars in breeding programs to extreme levels, but the cause of albinism has not as yet been determined.

In this study, we compared two barley cultivars producing contrasting rates of green regenerants in an isolated microspore culture. Both cultivars produced more than 100 plantlets per 100,000 microspores in total, however, the rate of green plantlets in cv. "Jersey" was $95 \%$ and in cv. "Mercada" only $5 \%$. In the presented study, the expression level of genes related to plastid development, chloroplast differentiation and photosynthesis, as well as plastome copy number and plastid ultrastructure during regeneration of androgenic embryos between 43 day and 65 day of culture were compared for both cultivars. The regenerated green plantlets of both cultivars showed a normal chloroplast development while the albino plantlets contained plastids arrested at the early stage of development. However, green regenerants of cv. "Jersey" contained ca. 30\% more chloroplasts than green plantlets of cv. "Mercada". Plastids of albino regenerants of both tested cultivars contained only prolamellar body or unarranged thylakoids membrane within plastids, in contrast to well development grana in chloroplasts of green androgenic plantlets. The expression level of plastid or nuclear-encoded genes related to photosynthesis was extremely reduced in albino plantlets in comparison to the green regenerants. Additionally, the expression level of plastid $r R N A s$ increased drastically during embryo regeneration in cv. "Jersey" which was not observed during regeneration in the cultivar "Mercada" producing mostly albino regenerants. Contrary to cv. "Jersey", cv. "Mercada" also showed no increase in the expression level of transcriptional factors regulating the expression of photosynthetic genes induced by light, such as $G L K 1$ and $G L K 2$, what was probably the consequence of an impaired expression of plastid $r R N A$.

This study was fully supported by the Polish Ministry of Agriculture and Rural Development grant No. HOR hn - 801-PB-7/15. 


\title{
The effect of zearalenone on the anther culture of wheat
}

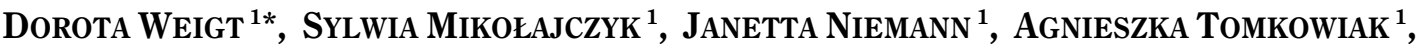 \\ DANUTA KURASIAK-POPOWSKa ${ }^{1}$, JOANNA ZYPRYCh-WALCZAK ${ }^{2}$, IDZI SiaTKOWSKI ${ }^{2}$ \\ ${ }^{1}$ Department of Genetics and Plant Breeding, University of Life Sciences, Poznań, Poland \\ ${ }^{2}$ Department of Mathematical and Statistical Method, University of Life Sciences, Poznań, Poland \\ *dorota.weigt@up.poznan.pl
}

Zearalenone (ZEA) is a mycotoxin produced mainly by fungi from the genus Fusarium, which are regular contaminants of cereal crops worldwide. Used in an appropriate concentration, it stimulates indirect regeneration and increases the frequency of embryo formation in maize pollination system [1]. The effect of ZEA was similar to the activity of auxins in in vitro cultures affecting the calli proliferation and cell differentiation. Due to these properties, ZEA was used in the experiment as a potential factor, which could decrease the genotypes' dependence in the anther culture of wheat by stimulating cells to divide and embryogenic structures to form. So far, ZEA has not been examined as a component of induction media in androgenesis.

The aim of the present study was to examine the influence of ZEA on the induction of embryogenic structures (ES), the formation of green plantlets (GP) and albino plantlets (AP) and the ploidy level (PL) in the anther culture of wheat.

Nineteen $\mathrm{F}_{1}$ hybrids of winter and spring wheat were used. All were from breeding crosses, obtained in the Department of Genetics and Plant Breeding, Poznań University of Life Sciences. Spikes were cold-treated at $4{ }^{\circ} \mathrm{C}$ for 1 week. The anthers were transferred onto a C 17 -inducing medium supplemented with $90 \mathrm{~g} / 1$ maltose and solidified by $6 \mathrm{~g} / \mathrm{l}$ gerlite. Six combinations of growth regulators and ZEA were applied:

1) $1.0 \mathrm{mg} / \mathrm{dm}^{3} 2,4-\mathrm{D}, 1.0 \mathrm{mg} / \mathrm{dm}^{3}$ dicamba (DIC), $0 \mu \mathrm{l} / \mathrm{dm}^{3} \mathrm{ZEA}$ (DIC-0),

2) $1.0 \mathrm{mg} / \mathrm{dm}^{3} 2,4-\mathrm{D}, 1.0 \mathrm{mg} / \mathrm{dm}^{3}$ dicamba (DIC), $100 \mu \mathrm{l} / \mathrm{dm}^{3}$ ZEA (DIC-1),

3) $1.0 \mathrm{mg} / \mathrm{dm}^{3}$ 2,4-D, $1.0 \mathrm{mg} / \mathrm{dm}^{3}$ dicamba (DIC), $200 \mu \mathrm{l} / \mathrm{dm}^{3}$ ZEA (DIC-2),

4) $1.5 \mathrm{mg} / \mathrm{dm}^{3} 2,4-\mathrm{D}, 0.5 \mathrm{mg} / \mathrm{dm}^{3}$ kinetin (KIN), $0 \mu \mathrm{l} / \mathrm{dm}^{3}$ ZEA (KIN-0),

5) $1.5 \mathrm{mg} / \mathrm{dm}^{3}$ 2,4-D, $0.5 \mathrm{mg} / \mathrm{dm}^{3}$ kinetin (KIN), $100 \mu \mathrm{l} / \mathrm{dm}^{3}$ ZEA (KIN-1),

6) $1.5 \mathrm{mg} / \mathrm{dm}^{3}$ 2,4-D, $0.5 \mathrm{mg} / \mathrm{dm}^{3}$ kinetin (KIN), $200 \mu \mathrm{l} / \mathrm{dm}^{3}$ ZEA (KIN-2).

600 anthers of each of the 9 genotypes were plated in every combination of grow regulators. A total of 34200 anthers were cultured and were incubated in the dark at $28^{\circ} \mathrm{C}$. Individual ES were transferred onto the regeneration medium MS supplemented with $0.5 \mathrm{mg} / \mathrm{dm}^{3} \mathrm{NAA}$, incubated in culture room at $24^{\circ} \mathrm{C}$ and sub-cultured every 3 weeks. GP were transplanted to soil and placed in a growth chamber. The ploidy level of the obtained plants was analyzed by laser flow cytometry. Haploid plants were treated using $0.05 \%$ colchicine solution.

The influence of ZEA on ES formation was significant. $200 \mu \mathrm{l} / \mathrm{dm}^{3}$ addition of ZEA to both DIC and KIN medium was most productive in the induction of microspores proliferation. The effectivity of ES formation ranged from $4.1 \%$ (ES per 100 plated anthers) on KIN-0 to $8.8 \%$ on DIC-2 medium. ZEA supplementation $\left(200 \mu 1 / \mathrm{dm}^{3}\right)$ had also a beneficial effect on GP formation increasing efficiency to $150 \%$ (GP per 100 plated anthers) on DIC-2 medium and to $176 \%$ on KIN-2 medium in comparison to Dic-0 and KIN-0 respectively. The influence of ZEA on AP formation and ploidy level was not significant. AP formation ranged from $0.5 \%$ (AP per 100 plated anthers) on KIN-0 and KIN-2 to $1.3 \%$ on DIC-0 and DIC-2. The addition of ZEA to the induction medium significantly increased the number of ES and GP, which however did not affect the AP formation and PL.

\section{References}

[1] Biesaga-Kościelniak J., Marcińska I., Wędzony M., Kościelniak J. (2003) Plant Cell Rep. 21: 1035-1039. 


\title{
Biotechnology in plant diversity conservation
}

\author{
Anna MikuŁa *, Karolina TomiczaK, MaŁgorzata Grzyb, WoJciech Tomaszewicz, Jan J. Rybczyński \\ Polish Academy of Sciences Botanical Garden - Center for Biological Diversity Conservation in Powsin, Poland \\ *a.mikula@obpan.pl
}

Biological diversity, that is the basis of human survival and economic development, is the variability within and between species and ecosystems. The anthropogenic pressure, the introduction of alien species, as well as domesticated species have dramatic effects on plant diversity, which is reflected in an increase in the number of threatened species. It has been estimated that one third of the global plant species are threatened in different level according to the International Union of Conservation of Nature (IUCN). The conservation of plant diversity is a global concern that is attempted to solve either by in situ or ex situ methods. Approximately 7.4 million germplasm accessions representing more than 16,500 plant species are currently conserved in 1750 genebanks worldwide, i.e. botanical gardens, field gene collections, seed banks, liquid nitrogen banks. Advances in biotechnology help to broaden the crop diversity, complement the ex situ preservation programs for plant biodiversity, and provide the new tools to improve conservation and management of plant diversity of crop, wild, and threatened species.

Biotechnological methods, especially those associated to in vitro culture and cryopreservation, support the multiplication, collection, and short- to long-term conservation of many plant species. Due to cell totipotency, almost any part of the plant is sufficient to regenerate a whole plantlets under the appropriate tissue culture conditions. It leads to the production of a new category of germplasm, such as shoot tips, somatic embryos, embryogenic tissue, cell lines with special attributes, and genetically transformed material. In vitro technique has been extensively applied for multiplication and regeneration of over 1000 different plant species, including many rare and endangered species. Their tissue culture systems of massive clonal multiplication can be used to further in vitro plant germplasm conservation under slow growth conditions, and to long-term cryostorage. Slow growth conservation bases on reducing the growth rates of the stored plant materials by modifying the growth media composition or storage under minimal growth conditions, such as, low temperature and dark incubation. Cryopreservation technique is the most effective approach for storage of plant cells, tissues, parts of organs and embryos at ultra-low temperature $\left(-196^{\circ} \mathrm{C}\right)$. It is a preservation method that enables plant genetic resources to be conserved safely, and cost-effectively. Although various cryopreservation methods based on the standard protocols, such as slow freezing, encapsulation/dehydration, vitrification, encapsulation/vitrification and droplet/vitrification are widely applied, the development of simple, reliable and cost effective method is still essential.

The aim of the talk is to present the current state of knowledge on the application of the biotechnological tools in the development of ex situ collections (both crops and endangered species) and their contribution toward an integrated system to conserve plant species. 


\title{
Large scale micropropagation of rare and endangered mosses
}

\author{
Oliwia Urbanowicz ${ }^{1 *}$, Paulina Dziubańska ${ }^{1}$, Jakub Fikowicz-Krośko ${ }^{2}$, Paulina Ćwiklińska ${ }^{3}$, \\ Klaudia Palenica ${ }^{1}$, Magdalena BaJor ${ }^{1}$, Zofia Ozymko \\ ${ }^{1}$ Pomeranian Science and Technology Park Laboratory Gdynia, Gdynia, Poland \\ ${ }^{2}$ Department of Biotechnology, Intercollegiate Faculty of Biotechnology, University of Gdansk and Medical University of Gdańsk, Poland \\ ${ }^{3}$ AIRA PAULINA CWIKLINSKA, Gdańsk, Poland \\ *o.urbanowicz@ppnt.gdynia.pl
}

Since 2009 BioLaboratory of Pomeranian Science and Technology Park Gdynia has worked on projects concerning ex situ conservation of bog mosses. Peatland protection is important because it improves air quality, provides a unique habitat and helps fight climate change. In the project, we have taken care of glacial relicts species such as: Tomenthypnum nitens, Helodium blandowii, Paludella squarrosa and Scorpidium scorpioides. Mosses covered by the project are associated with the calcareous groundwater. They occur in rare plant communities in Europe. Most of them are located in northern Poland. They are diffused in many habitats but a gradual decrease in the number of habitats is observed. We developed protocols for the establishment of $e x$ situ collections of sensitive plant groups where the initiation material is limited. Several methods of sterilizing the starting material and growing nutrient media were tested.

The aim of the study was the micropropagation and optimization of growth conditions protocols for four peatland plants: Tomenthypnum nitens, Helodium blandowii, Paludella squarrosa and Scorpidium scorpioides. Our research contributes to the conservation biology, as well as to the potential use of these bryophytes species in many biotechnology research.

The collections were made from various zones of biodiversity of the Pomeranian peat bogs located in Sulmino, Suleczyno, Mechowisko Radosc, Tuchom and Bialogora. Micropropagation of Tomenthypnum nitens, Helodium blandowii, Paludella squarrosa and Scorpidium scorpioides was initiated using a tissue. A series of experiments were carried out to assess the best sterilization technique for each tested plant. After sterilization, explants were cultured on a modified Murashige and Skoog basal medium (1/2 MS - 1/2 concentration macro elements), incubated in a photoperiod for $16 / 8$ hour at $22 \pm 2^{\circ} \mathrm{C}$ during 1-2 months. Afterwards, the cultures were transferred on the fresh growing medium every four months. In the next stage the best of cultures were transferred on a different medium for growing optimization (Hoagland, MS, 1/2 MS, RM, CHU(N6)). An optimized medium was described for each species. Results of optimization were adapted to increase the production of plant material: Tomenthypnum nitens, Helodium blandowii, Paludella squarrosa and Scorpidium scorpioides using a temporary immersion system (TIS). We present a utilization of a large-scale plant-breeding bioreactor, which has been adopted to multiply bryophytes biomass. Methods developed in in vitro culture have been wide used to increase the production of these plants in the future.

In this study, highly-efficient methods for micropropagation Tomenthypnum nitens, Helodium blandowii, Paludella squarrosa and Scorpidium scorpioides, have been developed. The micropropagation can be applied to produce these species of bog moss which are difficult to reproduce conventionally. Optimum growth parameters have been adjusted in order to achieve moss material ready to reintroduce to its natural range. Protocols used for the establishment of ex situ plant collections for conservation are applicable to a range of species with different ecological requirements. We are able to produce successful cultures from limited plant material. 


\title{
Use of tissue culture for ex situ conservation of the endangered Salicaceae species in the territory of Poland
}

\author{
Marzena Parzymies ${ }^{* 1}$, Magdalena Pogorzelec ${ }^{2}$, Barbara BanaCh-Albińska ${ }^{3}$, \\ Alicja ŚWISTOWSKA ${ }^{1}$, AgNIESZKa SZCZUROWSKA ${ }^{4}$, ARTUR SERAFIN ${ }^{5}$ \\ ${ }^{1}$ Department of Ornamental Plants, Dendrology and Landscape Architecture, University of Life Sciences in Lublin, Lublin, Poland \\ ${ }^{2}$ Department of Hydrobiology and Protection of Ecosystems, University of Life Sciences in Lublin, Lublin, Poland \\ ${ }^{3}$ Department of Zoology, Animal Ecology and Hunting, University of Life Sciences in Lublin, Lublin, Poland \\ ${ }^{4}$ Department of Plant Physiology, University of Life Sciences in Lublin, Lublin, Poland \\ ${ }^{5}$ Department of Environmental Engineering and Land Surveying, University of Life Sciences in Lublin, Lublin, Poland \\ * marzena.parzymies@up.lublin.pl
}

There are two relict boreal willows in Poland, Salix lapponum and Salix myrtilloides. These glacial relicts are present mainly on the eastern side of Vistula River. Both species have the status of endangered (EN, "Red Data Book") or vulnerable (EN by IUCN). Currently, their confirmed stands are located in Łęczna-Włodawa Lakeland in the Poleski National Park, in Biebrzański National Park and in Puszcza Knyszyńska.

The inventory conducted in the years 2010-2014 in the stands located in the Polesie Lubelskie Region described in the literature, proved an $80 \%$ decrease in a number of stands. A reduction in the number of specimens in the existing populations was also found. Therefore, there is no doubt that the species should be subjected to immediate conservation.

It was decided that the propagation $e x$ situ and the reintroduction of the obtained specimens would be the most effective method for active conservation. Due to a very low amount of plant material available and unsuccessful germination of seeds, micropropagation was chosen as the only possible way to obtain new plants.

The plant material for the in vitro culture initiation were fragments of shoots $5 \mathrm{~cm}$ in length, obtained from mother plants growing in natural stands. The explants were surface sterilized with different methods. It was noted that despite the disinfection method, most explants were contaminated, which might have resulted from the specific conditions of the mother plants habitat (Lake Bikcze), very high humidity and the closure of the peat bog in a basin. The two most effective methods were: 1) fungicide followed by water detergent solution followed by $70 \%$ ethanol followed by $1 \%$ solution of $\mathrm{NaOCl}$ and 2) water detergent solution followed by $70 \%$ ethanol followed by $0.1 \%$ of mercuric chloride. Those two methods allowed to obtain around $25 \%$ of contamination free explants.

The composition of the media affected the regeneration and multiplication of shoot explants. After preliminary studies, the shoot tissue cultures were cultivated on the MS (Murashige and Skoog, 1962) medium supplemented with benzyladenine (BA) in concentrations of 1.0 or $0.1 \mathrm{mg} / \mathrm{dm}^{3}$ and indolebutyric acid (IBA) in concentrations of 0.1 or $0.01 \mathrm{mg} / \mathrm{dm}^{3}$. It was observed that $S$. lapponum plants preferred a higher concentration of growth regulators while $S$. myrtilloides preferred the lower one. Due to a visible browning of the media and a low quality of shoots, the media were supplemented with activated charcoal (AC) in the concentration of $2.0 \mathrm{~g} / \mathrm{dm}^{3}$ or ascorbic acid (AA) in the concentration of $20 \mathrm{mg} / \mathrm{dm}^{3}$. The use of activated charcoal improved the quality of plants but decreased branching.

As the shoots rooted spontaneously, the rooting stage was omitted and the rooted shoots were transferred to a mixture of peat of 3.5-4.5 $\mathrm{pH}$, peat of $5.5-6.5 \mathrm{pH}$ and sand $(1: 1: 1 \mathrm{v} / \mathrm{v})$. The acclimatization process was conducted in high humidity conditions. As both willows are peat bog species, the pots with plants were partially submerged in water.

The obtained plants were reintroduced into natural stands in the third decade of May 2017. At present, they are being monitored. After a preliminary evaluation in the year 2018 it was noted that most of plants survived winter and are expected to grow. 


\title{
Is it possible to regenerate Pulsatilla patens (L.) Mill. from petioles using in vitro cultures?
}

\author{
ANNA ŹRÓBEK-SOKOLNIK \\ Department of Botany and Nature Protection, University of Warmia and Mazury in Olsztyn, Olsztyn, Poland \\ *a.zrobeksokolnik@uwm.edu.pl
}

Pulsatilla patens (L.) Mill. (syn. Anemone patens L.) is a species of a flowering, perennial herb plant of the family Ranunculaceae which is native to Europe, Russia, Mongolia, China, Canada and the United States. In Europe, the general population of $P$. patens is dramatically declining because of the destruction and disappearance of natural habitats, cessation of grazing, forest fires, and the habitat eutrophication. This is the reason for giving $P$. patens the status of a highly threatened species and a special concern of conservationists. Hence the inclusion of the species in the European Red List of vascular plants and in the national Red Data Books of all European countries where this plant occurs. In Poland, $P$. patens has been strictly protected since 1958. It should be noted that $P$. patens is an important plant not only from the biological and ecological perspective, but also due to its medicinal, ornamental, and cultural value, which gives another reason to study this species.

It is widely believed that $P$. patens reproduces only generatively. Little is known about the possibility of its reproduction in the vegetative way. For this reason, the aim of the study was to establish if it is possible to obtain in vitro regeneration of $P$. patens from petioles and thus obtain regenerants without affecting the natural populations. The experimental material were sterile young and tender petioles collected from $P$. patens plants growing in the garden collection of the Department of Botany and Nature Protection, Faculty of Biology and Biotechnology UWM in Olsztyn. The surface-sterilized explants were cut into $0.5-\mathrm{cm}$ pieces and arranged horizontally on the surface of the respective medium. The tested media were chosen based on literature data on the micropropagation of other Pulsatilla species, and own previous experiments. Callus induction and/or adventitious shoot regeneration process was conducted on MS medium supplemented with $0.5 \mathrm{mg} / \mathrm{dm}^{3}$ IAA and $0.25-2.0 \mathrm{mg} / \mathrm{dm}^{3} Z$ eatin $; 0.05 \mathrm{mg} / \mathrm{dm}^{3}$ IAA and $0.5 \mathrm{mg} / \mathrm{dm}^{3}$ Zeatin; $0.1 \mathrm{mg} / \mathrm{dm}^{3} \mathrm{NAA}$ with combination with 1.0 or $3.0 \mathrm{mg} / \mathrm{dm}^{3} \mathrm{BAP}$, Kin or $2 \mathrm{iP} ; 1.0 \mathrm{mg} / \mathrm{dm}^{3} 2,4-\mathrm{D}$ and $0.5 \mathrm{mg} / \mathrm{dm}^{3}$ BAP; 0.5 or $1.0 \mathrm{mg} / \mathrm{dm}^{3}$ 2,4-D and $0.5 \mathrm{mg} / \mathrm{dm}^{3}$ TDZ. The obtained callus was transferred to MS medium supplemented with $1.0 \mathrm{mg} / \mathrm{dm}^{3}$ BAP and $1.0 \mathrm{mg} / \mathrm{dm}^{3}$ PVP 10 for shoot regeneration. The obtained single shoots or shoot clumps were transferred to one of the variants of proliferation medium - MS medium supplemented with $0.05 \mathrm{mg} / \mathrm{dm}^{3}$ IAA and $0.5-1.5 \mathrm{mg} / \mathrm{dm}^{3}$ Zeatin; MS or WPM medium supplemented with $0.05 \mathrm{mg} / \mathrm{dm}^{3}$ IAA and $1.0 \mathrm{mg} / \mathrm{dm}^{3}$ BAP. The newly regenerated shoots (approximately $3-5 \mathrm{~cm} \mathrm{long}$ ) were separated, and then placed on a half-strength MS medium supplemented with $5.0 \mathrm{mg} / \mathrm{dm}^{3}$ a-naphthalene acetic acid (NAA) and $3.76 \mathrm{mg} / \mathrm{dm}^{3}$ riboflavin for root induction. After 5 days in dark conditions, test tubes with shoots were transferred to light (16-h photoperiod) for auxin peroxidation.

The morphogenetic response was observed only on MS medium with 2,4-D and $0.5 \mathrm{mg} / \mathrm{dm}^{3}$ BAP (callus and shoots); $0.5 \mathrm{mg} / \mathrm{dm}^{3}$ 2,4-D and $0.5 \mathrm{mg} / \mathrm{dm}^{3} \mathrm{TDZ}$ (callus); $1.0 \mathrm{mg} / \mathrm{dm}^{3} \mathrm{BAP}$ and $1.0 \mathrm{mg} / \mathrm{dm}^{3} \mathrm{PVP} 10$ (shoots from callus); and $0.05 \mathrm{mg} / \mathrm{dm}^{3}$ IAA and $0.5 \mathrm{mg} / \mathrm{dm}^{3}$ Zeatin (shoots). Shoot proliferation was observed on all tested media. The process of root formation was not induced under the conditions of our study.

This has been the first successful report of in vitro shoot regeneration of $P$. patens from petioles, but it is still necessary to establish optimal conditions for successful rooting of the obtained shoots. 


\title{
Cryopreservation of shoot tips serves Staphylea pinnata L. germplasm conservation
}

\author{
Bożena Szewczyk-TaraneK*, Barbara Prokopiuk, Monika Cioć, Bożena Pawlowska \\ Department of Ornamental Plants, University of Agriculture in Krakow, Kraków, Poland \\ *bozena.szewczyk-taranek@urk.edu.pl
}

Staphylea pinnata L. (Eng. common name - European bladdernut) is a rare and endangered shrub with ornamental value, growing in deciduous forests of southern Europe extending to Asia Minor [1]. This distinctive species is the only representative of Staphyleaceae in Europe with its northern range boundary found in southeastern Poland. Bladdernut is a termophilous calcicole, stoloniferous shrub growing up to $5 \mathrm{~m}$, with numerous sparsely branched stems. It blossoms in mid-May with a distinctive, pendent inflorescence and roundish, pearl-like, scented flowers. Weaver (1980) [3] estimated $S$. pinnata as the first-rate ornamental, deserving wider cultivation, due to its minimal maintenance and tremendous aesthetic value. $S$. pinnata sites are treated as natural, however, many modern localities of this plant are of anthropogenic origin. This plant used to be often found in household gardens in southern Poland due to the traditions of Carpathians tribes, folklore and vast ethnobotanical importance. However, the structure of plantings in Polish gardens has changed dramatically over the last decade, and bladdernuts are scarcely encountered nowadays.

The aim of the present experiment was to assess the suitability of cryopreservation using the droplet vitrification method in $S$. pinnata protection. Winter twigs of $S$. pinnata were collected in February from a local population in the deciduous forest near Tenczynek $\left(50^{\circ} 07^{\prime} 21.8^{\prime \prime} \mathrm{N} 19^{\circ} 37^{\prime} 03.0^{\prime \prime} \mathrm{E}\right)$, Krzeszowice Forestry District, of the KrakówCzęstochowa Upland, Poland. The permission allowing to research this plant which is under strict legal protection was obtained from the Regional Authority for Environmental Protection in Kraków.

For cryopreservation, the shoot tips were isolated from winter buds, and the scales covering the buds were removed after their meristem exposure. The explants were cryopreserved using the droplet vitrification method, according to Pawłowska and Szewczyk-Taranek (2014) [2], after testing the LS, PVS2 treatment time (10, 20 and $30 \mathrm{~min}$ ). After thawing, the explants were placed on a medium containing $50 \% \mathrm{MS}, 0.3 \mathrm{M}$ sucrose and $0.5 \%$ BioAgar in the dark. The shoot tips were transferred to the MS shoot multiplication medium supplemented with BA ( $5 \mu \mathrm{M})$ and NAA $(0.5 \mu \mathrm{M})$ after $24 \mathrm{~h}$ and maintained in the dark during the first week; then the intensity of light (PPFD) was gradually increased to $30 \mu \mathrm{M} / \mathrm{m}^{2}$ s after the second week. The survival of the shoot tips after cryopreservation was assessed 15 days after thawing, based on visual observations. The percentage of explants with developed leaves and stems represented the regeneration rate and was calculated 35 days after thawing.

The method applied can also be used for $S$. pinnata shoot tip protection in liquid nitrogen. The highest survival rate after cryopreservation was 59\%, when the PVS2 treatment time was 10 minutes. Almost all explants that survived cryopreservation regenerated (54\% regeneration rate). A prolonged PVS2 treatment (20, 30 minutes) suppressed the survival and regeneration rate.

\section{References}

[1] Meusel H., Jäger E.J. (1989) In: Plants Systematics and Evolution 162, Springer-Verlag: 315-329.

[2] Pawłowska B., Szewczyk-Taranek B. (2014) Sci. Hortic. Amsterdam 168: 151-156.

[3] Weaver R. (1980) Arnoldia 40(2): 76-93.

This study was supported by the Polish Ministry of Science and Higher Education: DS 3500/KRO/2017. 


\title{
Smoke-derived karrikin - a new natural compound regulating the growth and development of plants
}

\author{
JAN KĘPCZYŃSKI \\ Department of Plant Physiology and Genetic Engineering, Faculty of Biology, University of Szczecin, Szczecin, Poland \\ * jankepcz@wp.pl
}

Plant-derived smoke and smoke water (SW) were found to stimulate seed germination of plants from fire-prone areas. Dormant and non-dormant seeds, including those of agricultural weeds and crop plants, from fire-free environments turned out to be sensitive to smoke as well. Seeds of 1200 species in more than 80 genera were found to positively respond to smoke. Six karrikins ("karrik" meaning smoke in Australian Aboriginal language), KAR1 KAR6, a family of closely related small organic compounds were identified in the plant-derived smoke. These compounds consist of five-membered butenolide rings and six-membered pyran rings. Karrikins are by-products of pyrolysis of carbohydrates such as cellulose and simple sugars. KAR2-KAR6 concentrations in smoke were much lower than those of KAR1.Thus, KAR1 was considered to be the major factor responsible for the stimulatory effect of smoke. The compound was found to be active at very low concentrations $\left(10^{-10}-10^{-7} \mathrm{M}\right)$ and neither toxic or genotoxic at $3 \times 10^{-10}-10^{-4} \mathrm{M}$. KAR1 was shown to stimulate seed germination in fire-prone environments in hemiand holo-parasitic seeds and in several Australian Asteraceae species. It is also an effective agent in stimulating seed germination of a broad spectrum of both monocotyledonous and dicotyledonous weed species. Seeds of some crop plants such as lettuce, tomato, okra, bean, maize, and rice responded positively to KAR1. The compound is able to increase both, the rate and percentage of seed germination. Moreover, and importantly, KAR1 allows seeds to germinate at sub- and supra-optimal temperatures, and also at a low water potential. It can be used as a priming agent, e.g., for tomato seeds. However, seeds of some species proved not sensitive to SW or KAR1, but responded positively to KAR1 or SW respectively. Karrkin was reported to facilitate cotyledon expansion and chlorophyll accumulation in Arabidopsis thaliana seedlings, and also to increase seedling vigour and stress tolerance of several crop species. KAR1 was also found to shorten the somatic embryo development duration. Most studies on karrikin action mechanisms focused on Avena fatua caryopses and $A$. thaliana seeds and seedlings. The stimulatory effects of KAR1 and gibberellin on germination of dormant $A$. fatua caryopses were found to be similar and related. The expression of KAR1 stimulating effects requires endogenous gibberellins. KAR1 was found to stimulate degradation of ABA, the most important factor for induction and maintenance of dormancy. Although ethylene alone is not able to remove dormancy, it is required for caryopses to respond to KAR1. The mechanism of KAR1-induced dormancy release and $A$. fatua caryopsis germination involves regulation of the ROS-antioxidant status both in embryos and in aleurone layers as well as the cell cycle activity. So far, information on the mechanism of karrikin effects at the molecular level has been provided by research on $A$. thaliana seeds and seedlings. The signalling mechanism of karrikins and strigolactones, synthesized in plants, both containing 3-methyl-butenolide ring, was found to be highly similar. Moreover, the signalling pathways of karrikins and strigolactones remind those of GAs where the receptors bind to FBOX proteins in the SCF E3 ubiquitin ligase complexes to regulate the degradation of transcriptional repressors. The discovery of karrikins creates new possibilities for improving seed germination, establishment of horticultural and agricultural plant seedling and revegetation. It also provides opportunities for studying the physiological roles and the mode of mechanism action of new natural compounds at important early stages of plant development. 


\title{
Meta-Topolin as an alternative to 6-Benzyladenine during micropropagation
}

\author{
Karolina NowaKowska *, Andrzej Pacholczak \\ Department of Ornamental Plants, Warsaw University of Life Sciences - SGGW, Warszawa, Poland \\ *karolina_nowakowska@sggw.pl
}

An increasing demand for plant material produced in tissues culture and a growing importance of the in vitro methods as research tools the new effective solutions are sought for in order to obtain a better quality plant material. The type and concentration of growth regulators - mainly cytokinins and auxins - included in a multiplication culture medium is a decisive factor for the success of its propagation. One of the less common and relatively new cytokinins is meta-Topolin (mT) used alternatively to the most popular 6-benzyladenine (BA). Till now - because of its availability and a low price - BA has been the most frequently used growth regulator in the micropropagation of most plant species. However, there are several drawbacks related to its use: it may cause shoot vitrification or negatively affect rooting of microcuttings. Therefore, investigations on the effects of another cytokinin - meta-Topolin - on the regeneration in vitro of many plants have recently been carried out. Till now, it has been proved that the application of $\mathrm{mT}$ eliminates most of problems resulting from the use of $\mathrm{BA}$, improves regeneration and stimulates rhizogenesis in microcuttings.

The aim of the experiments was to compare the effect of the commonly used BA and a less known mT analogue on the regeneration in explants of Daphne mezereum and D. mezereum "Alba" and on the contents of several compounds in microcuttings. February dafne (D. mezereum L.) is legally protected in Poland. Till now no effective method of its propagation has been developed. The commercially used generative method, i.e. seed sowing does not always give good results as splitting of plant traits occurs in seedlings. In the experiments, the culture media such as MS (Murashige and Skoog) and WPM (Woody Plant Medium) were used. They were supplemented with $0.1 \mathrm{mg} / \mathrm{dm}^{3}$ auxin 1-Naphthylacetic acid (NAA) and $1.0 \mathrm{mg} / \mathrm{dm}^{3} \mathrm{mT}$ or $2.5 \mathrm{mg} / \mathrm{dm}^{3}$ BA. After 6 weeks of culture the following observations were made: the percentage of regenerating explants, the number of microshoots per explant and the shoot length. The biochemical analyses of assimilation pigments (chlorophyll $a+b$ and carotenoids), the total soluble and reducing sugars, soluble proteins and free amino acids were performed. The results showed that metaTopolin affected shoot regeneration better or in a comparable way to BA. The percentage of regenerating explants was high on media with both cytokinins, however, in the case of regeneration of the dafne species on MS, metaTopolin increased the number of regenerating explants by $10 \%$ relative to BA. The largest number of microshoots per explant was obtained on MS supplemented with $1.0 \mathrm{mg} / \mathrm{dm}^{3} \mathrm{mT}$ and $0.1 \mathrm{mg} / \mathrm{dm}^{3} \mathrm{NAA}$, while the longest shoots were produced on WPM enriched with $\mathrm{mT}$. On the MS medium there were no differences in shoot length between microcuttings cultured in presence of BA or $\mathrm{mT}$. The biochemical analyses showed a positive effect of meta-Topolin as compared to benzyladenine. The highest amount of chlorophyll $a+b$ was found in microcuttings cultured on MS or WPM supplemented with $1.0 \mathrm{mg} / \mathrm{dm}^{3} \mathrm{mT}$ and $0.1 \mathrm{mg} / \mathrm{dm}^{3} \mathrm{NAA}$, while that of carotenoids - in microshoots from WPM with $\mathrm{mT}$. The contents of reducing sugars, soluble proteins and free amino acids were the highest in plantlets cultured on either medium (MS or WPM) enriched with $1.0 \mathrm{mg} / \mathrm{dm}^{3} \mathrm{mT}$ and $0.1 \mathrm{mg} / \mathrm{dm}^{3} \mathrm{NAA}$. 


\title{
In vitro regeneration of adult Pinus sylvestris L. trees
}

\author{
IWONA SZYP-BOROWSKa, KRYSTYNA SZCZYGIEE, JAN KOWALCZYK \\ Department of Silviculture and Forest Tree Genetics, Forest Research Institute, Raszyn, Poland \\ *I.Szyp@ibles.waw.pl
}

The propagation of adult conifer trees by tissue culture has been studied for the last twenty years, but problems related to the juvenile to adult phase change of trees have limited the practical applications of these tissue culture procedures. In vitro regeneration is less successful from explants of mature woody plants than from their juvenile counterparts because numerous physiological and biochemical changes are implicated in the juvenile to adult phase transition. However, in vitro propagation of selected mature trees could be a powerful tool to improve forestry management and breeding programs. Vegetative propagation of selected trees offers a rapid way of generating clonal planting stocks for reforestation programs, woody biomass production and conservation of elite tree. This study describes a micropropagation protocol for the in vitro propagation of mature Scots pine trees. In Europe, Scots pine (Pinus sylvestris L.) is one of the most commercially important conifer species. Dormant shoot buds, which had not started to elongate, were collected from adult $P$. sylvestris trees (>10 years old). Buds were collected twice in February and April from the Forest District Syców and Nurzec. For surface disinfection the explants were surface sterilized in: water plus $0.01 \%$ Tween- 20 for $15-20 \mathrm{~min}$, followed by $70 \%$ ethanol for $10 \mathrm{~min}$ and mercury chloride $\left(\mathrm{HgCl}_{2}\right)(0.1 \% \mathrm{w} / \mathrm{v})$ for 25 min under constant agitation. The explants were rinsed 3 times in autoclaved distilled water. The sampled buds were cultured on two types of culture media (Murashige and Skoog and Woody Plant Medium) supplemented with cytokinins at different concentrations: $B A 1.0 \mathrm{mg} / \mathrm{dm}^{3}$ and $\mathrm{mT} 1.0 \mathrm{mg} / \mathrm{dm}^{3}, 3.0 \mathrm{mg} / \mathrm{dm}^{3}$, $6.0 \mathrm{mg} / \mathrm{dm}^{3}$. Shoot buds cultured on MS medium presented the lowest organogenic response perhaps as a result of the relatively high nitrate concentration when compared to the WP media. For this reason we finally used WPM as a basal medium. Upon bud sprouting, the explants were placed on the elongation media supplemented with $0.2 \%$ $(\mathrm{w} / \mathrm{v})$ activated charcoal. A positive effect of activated carbon on in vitro cultures was recognized in earlier studies. According to some authors this is probably the result of the absorption of phytohormones and phenol substances decreasing morphogenesis. When the elongating needle fascicles were more than $2 \mathrm{~cm}$ in length, they were cut and transferred to the rooting medium. A half concentrated WPM medium was supplemented with $10 \mu \mathrm{M}$ naphthaleneacetic acid (NAA) and $20 \mathrm{~g} / \mathrm{dm}^{3}$ glucose. The microshoots were kept in this medium for 1 week in the dark, and were subsequently transferred to the half WP supplemented with $10 \mathrm{~g} / \mathrm{dm}^{3}$ glucose cultured under light. A correct rooting process (prior to or during acclimatization) determines the success of the acclimatization phase and thus of the plant establishment in the field. In vitro rooting was, however, difficult to achieve and could only be induced at low rates. This protocol is a successful and efficient biotechnological approach to the micropropagation of adult $P$. sylvestris trees. Future research will focus on the improvement of the rooting rates. 


\title{
The effect of trophic stress on embryo sac development in pistils of Fagopyrum esculentum (Moench.) in in vitro conditions
}

\author{
Agnieszka PŁażeK ${ }^{1 *}$, Aneta SŁomka ${ }^{2}$, Franciszek Dubert ${ }^{3}$, PrzemysŁaw Kopeć ${ }^{3}$, \\ Marta Hornyák ${ }^{1}$, Michą DZIURKa ${ }^{3}$, JaKub PaStuszaK ${ }^{1}$ \\ ${ }^{1}$ Department of Plant Physiology, Faculty of Agriculture and Economics, University of Agriculture in Krakow, Kraków, Poland \\ ${ }^{2}$ Department of Plant Cytology and Embryology, Jagiellonian University, Kraków, Poland \\ ${ }^{3}$ The Franciszek Górski Institute of Plant Physiology, Polish Academy of Sciences, Kraków, Poland \\ *rrplazek@cyf-kr.edu.pl
}

Buckwheat is a plant cultivated due to the very good chemical composition of the seeds, and especially because of the very high lysine content and lack of gluten. Buckwheat nectar is a source of valued honey containing many healthy substances. For a long time there has been a decline in buckwheat production in all countries due to low seed production. The major causes of low buckwheat productivity occurring in its reproductive system might include: 1) self-incompatibility caused by dimorphic heterostylism, i.e. the occurrence of two types of flowers differing in the length of the pistils and anthers (Pin and Thrum type), 2) short vitality and ability to fertilize of a single flower, 3) incomplete reproductive organ, mainly in the female. Buckwheat is a plant blooming throughout the whole vegetative period (non-selfending), which can cause strong competition for assimilates. between immature seeds and production of new flowers. The results obtained in the previous project funded by the Ministry of Agriculture and Rural Development indicate that the more flowers a plant produces, the greater their abortion, and the number of flowers correlates negatively with the number of seeds. The number of degenerated embryo sacs in the studied plants ranged from 10 to $30 \%$, while the pollen viability was very high. These results indicate that degenerative female gametophytes rather than developmental disorders of microspores are the cause of the lack of fertilization. Degeneration of embryos and embryo sacs may also be due to their genotypic characteristics, high temperature during flowering and "starvation", i.e. high competition for assimilates between embryos and still produced flowers.

The goal of the presented experiment was to study the trophic stress effect on the megasporo- and megagametophytogenesis of common buckwheat. The experiment was performed on isolated pistils in vitro conditions. Pistils were isolated from green buds of control plants grown at $20^{\circ} \mathrm{C}$ and photoperiod of $16 \mathrm{~h} / 8 \mathrm{~h}$ day/night, and put on the two different media providing all the necessary compounds for embryological development (control). One part of pistils was put on the media containing half of the control medium content. Whole pistils of size $0.5-1.5 \mathrm{~mm}$ were sterilized in $70 \%$ ethanol for $30 \mathrm{~s}$ and $2 \% \mathrm{NaClO}$ for $10 \mathrm{~min}$, washed three times in sterile distilled water and put on control media: 1) MS (Murishage and Skoog, 1962) with B5 vitamin $\left(2.0 \mathrm{mg} / \mathrm{dm}^{3}\right)$, glutamine $\left(0.1 \mathrm{~g} / \mathrm{dm}^{3}\right)$, kinetin $\left(1.0 \mathrm{mg} / \mathrm{dm}^{3}\right)$, gibberelic acid $\mathrm{GA}_{3}\left(1.0 \mathrm{mg} / \mathrm{dm}^{3}\right), 30 \mathrm{~g} / \mathrm{dm}^{3}$ sucrose and solidified with agar $\left(6.0 \mathrm{~g} / \mathrm{dm}^{3}\right)$ $(\mathrm{pH}=5.6)$, or 2) $1 / 2 \mathrm{MS}$ 6-benzylaminopuryne (BAP) $\left(1.0 \mathrm{mg} / \mathrm{dm}^{3}\right)$, 1-naftylacetic acid (NAA) $\left(0.1 \mathrm{mg} / \mathrm{dm}^{3}\right)$, sucrose $\left(30 \mathrm{~g} / \mathrm{dm}^{3}\right)$, and agar $\left(6.0 \mathrm{~g} / \mathrm{dm}^{3}\right)$. Trophic stress was evoked on adequate half-strength media solidified with the same amount of agar as the control ones.

Dishes with isolated pistils were placed in the same thermal and light conditions as donor plants. The material for embryological analysis was collected every two days (during ten days). The detail results will be collected in August 2018.

The Project is funded by the National Centre of Science (2017/25/B/NZ9/00148). 


\title{
Terpenoids in flax response to fusariosis
}

\author{
Aleksandra Boba, Jan Szopa-Skórkowski, Anna Kulma \\ Faculty of Biotechnology, University of Wrocław, Wrocław, Poland \\ *aleksandra.boba@uwr.edu.pl
}

Flax is a valued crop, which delivers such raw products like oil and fibre. Currently, thanks to modern technologies, virtually all its parts can be utilized, including those that were thought to be by-products, that is shives or seedcakes, which are the main source of bio-active compounds. Although the conditions for flax cultivation are ideal in our country, its acreage decreases. It is connected with its susceptibility to diseases, among which fungi of Fusarium genus are most dangerous, one of them being $F$. oxysporum which is a flax-specific pathogen.

Depending on the pathogen type plants have developed various defense mechanisms, which involve different compounds and processes to stop the infection. According to the literature, PR proteins, phenylpropanoids or poliamines, are all involved in flax response to fusariosis while there are only few reports on the role of terpenoids.

The main aim of the study was the assessment of the role of selected terpenoids in the response to infection with fungi of the Fusarium genus. As terpenoids are a highly diverse group of secondary metabolites, a particular analysis of three groups of these compounds: carotenoids, tocopherols and sterols was performed.

In flax seedlings treated with a pathogen, the expression levels of previously identified genes were analyzed and metabolites were assayed. Moreover, the influence of pure compounds (analytical standards) on pathogen growth was investigated.

The obtained results indicate that in the case of a Fusarium infection the non-mevalonate pathway is activated in flax, which leads to the synthesis of such compounds as carotenoids and tocopherols. The synthesis of phytosterols, deriving from the mevalonate pathway, slightly decreased, which was evidenced by measuring the levels of the sterols and expression of genes related to their biosynthesis. The content of tocopherols increased slightly during the infection, which relates to the growth in the expression level of the adequate genes. However, $\alpha$-tocopherol did not show any inhibitory effect on the pathogen growth. An increased synthesis of tocopherols can thus be connected with the necessity of quenching free radicals produced during the infection. The level of carotenoids in the course of the infection increased initially to drop down slightly below the control level during the following hours. The carotenoids, similarly to tocopherol, did no inhibit pathogen growth, though they influenced its metabolism. Therefore, the level of expression of the genes was linked to the carotenoid oxidative cleavage and ABA synthesis. Expression of $n c e d 3$ and $n c e d 6$ genes, responsible for the carotenoid oxidative cleavage to the precursors of abscisic acid, was significantly increased. Equally, the expression of genes directly connected with ABA synthesis was higher. This was reflected in the content of ABA, which was increasing from the very beginning of the infection. The abscisic acid is considered a signal molecule, which triggers appropriate defense mechanisms in plants, similar to salicylic or jasmonic acids. About $10 \%$ of the genes in the plant genome possess cis-regulatory elements sensitive to $\mathrm{ABA}$ in their promoter sequences. Two of such genes are callose synthase pmr4, and chitinase 10 whose participation in response to infection was confirmed in many cases. Its expression level, as well as callose content were determined. These values increased during the infection.

The obtained results indicate that within the terpenoid biosynthesis pathway the route leading tothe abscisic acid synthesis plays a key role in the response to Fusarium infection. The increased content of ABA in the cell triggers different resistance mechanisms, including the synthesis of callose, which contributes to the structure of papillae - a natural barrier to pathogen, and the synthesis of chitinase, which breaks down the glycosidic bonds in fungal chitin. 


\title{
Inoculation of Populus nigra "Italica" and $P . x$ canescens microplants with mycorrhizal fungi increase their tolerance to salt stress
}

\author{
MAGDALENA KULCZYK-SKRZESZEWSKA ${ }^{\text {, }}$, BARBARA KIELISZEWSKA-ROKICKA ${ }^{1}$, KRYSTYNA BOJARCZUK ${ }^{2}$ \\ ${ }^{1}$ Kazimierz Wielki University in Bydgoszcz, Bydgoszcz, Poland \\ ${ }^{2}$ Institute of Dendrology Polish Academy of Sciences in Kórnik, Kórnik, Poland \\ *mkulczyk@ukw.edu.pl
}

Populus spp. can develop dual mycorrhizal associations with ectomycorrhizal fungi (ECMF) and arbuscular mycorrhizal fungi (AMF), however variable degrees of ECM and AM colonization have been observed in roots of various species and hybrids. Soil salinity is an important major abiotic factor that significantly reduces global plant production. Symbiotic associations with ECM and AM fungi can be modified by environmental conditions. Species and varieties of Populus are able to adapt to adverse natural and anthropogenic environmental conditions, including salt stress, which makes them good candidates for growing on degraded soils. In vitro or ex vitro biotization of microplants with mycorrhizal fungi and/or symbiotic bacteria enables to surmount the problems of acclimatization microplants to ex vitro conditions. Mycorrhizas improve plant growth and survival by enhancing water and nutrient uptake and protecting the plant against environmental stresses, including salinity. Micropropagated Populus nigra "Italica" (Lombardy poplar) and $P$. x canescens (grey poplar) were maintained on modified half-strength MS medium (Murashige and Skoog, 1962) supplemented with plant hormones $\left(0.1 \mathrm{mg} / \mathrm{dm}^{3}\right.$ BAP and $\left.0.05 \mathrm{mg} / \mathrm{dm}^{3} \mathrm{NAA}\right)$. To this medium various levels of $\mathrm{NaCl}$ were added. Microplants of $P$. nigra "Italica" were more sensitive to osmotic and ion stresses than $P$. $x$ canescens microplants. At higher salt concentrations a negative relation between the period of salt treatment and the plants survival was recorded. Mycelia of ECM fungi (Paxillus involutus (Batch) Fr and Hebeloma crustuliniforme (Bull.) Quel.) were isolated from fungal sporocarps and were cultivated in Petri dishes on an agar medium (Pachlewski and Pachlewska, 1974). The inoculum from the AM fungus Rhizophagus irregularis (syn. Glomus intraradices Schenck and Smith (isolate BEG 87) was propagated with maize (Zea mays L.) for 4 months in pots filled with a sterilized mixture of soil and quartz sand $(1: 1, \mathrm{v} / \mathrm{v})$. The effects of inoculation with ectomycorrhizal (ECM) fungi ( $P$. involutus, H. crustiliniforme) and an arbuscular mycorrhizal fungus (AMF) $R$. irregularis) on Populus nigra "Italica" and $P . x$ canescens microplants under salt stress were studied. The coculture of the ECM fungi with microplants resulted in an altered root morphology, however no significant impact on the growth of shoots was observed. Microplants of $P$. nigra "Italica" and $P$. $x$ canescens micropropagated in vitro and inoculated ex vitro in sterile soil with ECM fungus $P$. involutus, AM fungus Rhizophagus irregularis or noninoculated were acclimatized for 8 weeks in a growth-chamber. Then the plants were watered for 2 weeks with $150 \mathrm{mM} \mathrm{NaCl}$ solution. The beneficial influence of mycorrhizal fungi on the survival of both Populus cultivars under salt stress was observed. The ECM fungus promoted the growth of P.x canescens and the AM fungus had no effect. ECM and AM inoculation of $P$. nigra "Italica" resulted in the reduction of the growth of the plantlets. After 4 months, the effectiveness of inoculation was verified. The root systems of both Populus clones were surrounded by septic hyphae of a mycorrhizal mycelium, however no ECM structures on the roots of the plantlets were found. The results showed that the presence of mycelium of ECM fungi in the rhizosphere stimulated the growth of poplar plantlets despite the absence of functional ECM structures on the roots. 


\title{
Effect of photosynthetically active radiation (PAR) and white LED light on the growth and rosmarinic acid production of Perovskia spp. in in vitro cultures
}

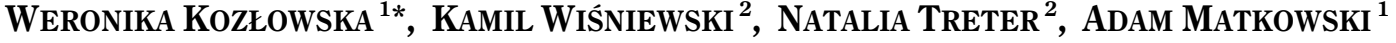 \\ ${ }^{1}$ Department of Pharmaceutical Biology, Wroclaw Medical University, Wrocław, Poland \\ ${ }^{2}$ Student Scientific Club No. K76, Department of Pharmaceutical Biology, Wroclaw Medical University, Wrocław, Poland \\ *weronika.kozlowska@umed.wroc.pl; kamil.wisniewski000@gmail.com
}

Perovskia is a genus of sub-shrubby, deciduous perennials native to central Asia, Pakistan, Afganistan and Iran. Thanks to its blue-purple inflorescence and greyish-green leaves it is used as an ornamental plant. The genus Perovskia belongs to the family Lamiaceae and is closely related to genus Salvia and Rosmarinus. It contains several species, from which Perovskia abrotanoides and Perovskia atriplicifolia are of greater importance.

In Iranian ethnopharmacology $P$. atriplicifolia is used as an anti-inflammatory and antiparasitic agent as well as a spice. Folk medicine exploits $P$. abrotanoides roots as a remedy against leishmaniasis while leaves are used as an antiseptic and anti-inflammatory agent in painful urination.

The herb of Perovskia spp. contains phenolic acids such as rosmarinic and caffeic acids. Rosmarinic acid is known to have antiviral, antibacterial and anti-inflammatory properties.

Six-week-old in vitro-germinated seedlings were used for the establishment of shoot cultures. Shoot tips were transferred to a proliferation medium every 6 weeks. Micropropagation was carried out on the basal (MS) medium which was supplemented with a single cytokinin - kinetin (KIN) or in combination with auxin - indole-3-acetic acid (IAA). Plant growth regulators (PGR) were added in a series of concentrations: $1,5,10 \mu \mathrm{M}$ for kinetin or 1, 5, $10 \mathrm{M}$ kinetin with $0.5 \mu \mathrm{M}$ IAA each. The cultures were maintained at $25 \pm 2^{\circ} \mathrm{C}$ under a $16 / 8 \mathrm{~h}$ (light/dark) photoperiod with two types of LED illumination: lamps emitting white light $(8000-10000 \mathrm{~K})$ and lamps emitting the light of the photosynthetically active radiation range (PAR): $730 \mathrm{~nm}, 660 \mathrm{~nm}, 630 \mathrm{~nm}, 610 \mathrm{~nm}, 460 \mathrm{~nm}, 430 \mathrm{~nm}$. The photon ux density was $180 \mu \mathrm{M} / \mathrm{m}^{2}$ s. Lyophilized plants were used for further analyses. Methanolic extract (water: methanol, $4: 1)$ of $P$. atriplicifolia and $P$. abrotanoides herb was analyzed to determine the content of phenolic acids.

The influence of plant growth regulators and the type of illumination on plant development was measured. The data regarding the length of main shoot, the number and length of axillary shoots per explant, the number of nodes per explant, the appearance of roots and the appearance of callus formed at basal parts of explants were recorded. The highest axillary bud break for both species $P$. atriplicifolia and $P$. abrotanoides was observed on the media with $1 \mu \mathrm{M}$ KIN and IAA $0.5 \mu \mathrm{M}$ under white light and KIN $10 \mu \mathrm{M}$ under PAR. The content of rosmarinic acid is a factor that highly corresponds to the variability of culture conditions. Its concentration ranges from $0.84 \mathrm{mg} / \mathrm{g} \mathrm{DW}$ on $5 \mu \mathrm{M}$ KIN to $6.2 \mathrm{mg} / \mathrm{g}$ DW on $1 \mu \mathrm{M}$ KIN with IAA for $P$. atriplicifolia in white light. The content of rosmarinic acid in plants cultivated in white LED light was higher than in plants cultivated on PAR. This phenomenon was observed for both species.

P. atriplicifolia and P. abrotanoides are convenient and responsive plants for in vitro experiments and can be used as a non-model system for investigations into the regulation of plant development and the metabolism of phenylpropanoids. 


\title{
Comparison of grass pea seedlings response to osmotic and chemical stress in in vitro conditions
}

\author{
Barbara PiWowarczyK, Krzysztof Tokarz, WoJciech MaKowski, IZabela WyEUPEK \\ Institute of Plant Biology and Biotechnology, Agriculture University of Krakow, Kraków, Poland \\ barbara.piwowarczyk@urk.edu.pl; krzysztof.tokarz@urk.edu.pl; wojtek.makowski.1305@gmail.com; polazi@onet.eu
}

The efficiency of plant production around the world is subject to ever greater limitations resulting from the abiotic stress, particularly drought and salinity, due to their high impact and wide distribution. Understanding mechanisms of plant tolerance for osmotic and chemical stress is fundamental for maintaining high crop productivity. Soil drought often occurs in combination with physiological drought, the latter one causes chemical stress due to high concentration of ions. Hence it is often assumed that acclimatization of plants to salinity and drought covers the same mechanisms. Grass pea (Lathyrus sativus L.) is a legume plant with extraordinary tolerance/resistance to abiotic stressors. It has capacity to withstand excessive drought and moderate salinity [1].

The issue whether acclimatization strategies of grass pea seedlings to osmotic and chemical stress have a similar basis on the physiological level was the aim of presented study.

Disinfected seeds of two Polish grass pea cultivars ("Derek" and "Krab") were laid on a standard germination medium with addition of different stress factors ( $\mathrm{NaCl}$ and $\mathrm{PEG}$ ). The concentration of $\mathrm{NaCl}$ and $\mathrm{PEG}$ was adjusted to create the osmotic potential of the medium on the level of $0.0 \mathrm{MPh}$ (control), $-0.25 \mathrm{MPh},-0.45 \mathrm{MPh}$ and $-0.65 \mathrm{Mph}$. After 7 days of culture, the germination rate and the seedling emergence rate were assessed. After 14 days of culture, the morphological parameters such as the length of the shoots and roots as well as the shoot and root dry weight were evaluated. Different biochemical analyses were also conducted on the collected material (shoots and roots separately), including the concentration of photosynthetic pigments, phenolic compounds, malonylodialdehyde (MDA), soluble and insoluble sugars, 3-N-oxalyl-2,3-diaminopropanoic acid ( $\beta$-ODAP) and activity of antioxidant enzymes.

In both cultivars, the highest osmotic potential $(-0.65 \mathrm{MPa})$ created by $\mathrm{NaCl}$ significantly decreased the germination and seedling emergence rates while that created by PEG decreased only the seedling emergence rate. "Derek" seedlings growing on the media with PEG were much smaller (shorter roots and shoots) than the ones growing on $\mathrm{NaCl}$ media. In turn, "Krab" seedlings from PEG treatment had only smaller shoots compared to seedlings from $\mathrm{NaCl}$ treatment. Dry weight increased considerably both in shoots and roots of both cultivars' seedlings cultivated on the media with PEG in comparison to seedlings from $\mathrm{NaCl}$ media. Moreover, the reduction of photosynthetic pigments concentration was greater in seedlings of "Derek" and "Krab" growing on the media with PEG than with $\mathrm{NaCl}$. The accumulation of phenolic compounds was higher in roots of seedlings from the media with lower $\mathrm{NaCl}$ concentrations than PEG and in shoots from the media with lower PEG concentrations than NaCl. MDA concentration increased in "Krab" but decreased in "Derek" seedlings from PEG treatment in comparison to seedlings from $\mathrm{NaCl}$. The concentration of sugars increased mostly in the shoots of PEG treated seedlings when compared to $\mathrm{NaCl}$ and in the roots of $\mathrm{NaCl}$ treated "Derek" seedlings when compared to PEG. $\beta$-ODAP content was higher in shoots of "Derek" and "Krab" seedlings and in roots of "Derek" seedlings from the media with higher PEG concentration than in the ones from $\mathrm{NaCl}$ media. The activity of peroxidase was greater in shoots of PEG treated "Derek" compared to $\mathrm{NaCl}$ and in roots of $\mathrm{NaCl}$ treated "Krab" compared to PEG.

The presented results showed that drought stress induced a resistance mechanism in the whole plant which consisted in the growth rate limitation for the benefit of the synthesis of secondary metabolites, while salinity stress induced mechanisms of osmotic (sugar concentration increase) and chemical (phenols accumulation) stresses response only in the roots.

\section{Reference}

[1] Campbell C.G. (1997) Grass pea, Lathyrus sativus L. Bioversity International, vol. 18. 


\title{
Molecular, biochemical and phenotypic evaluation of Lamprocapnos spectabilis (L.) Fukahura plantlets recovered from shoot tips cryopreserved via encapsulation - vitrification technique
}

\author{
DARIUSZ KuluS \\ Faculty of Agriculture and Biotechnology, UTP University of Science and Technology in Bydgoszcz, Bydgoszcz, Poland \\ *dkulus@gmail.com
}

Lamprocapnos spectabilis (L.) Fukuhara (syn. Dicentra spectabilis; the bleeding heart) is a popular perennial potted plant cultivated in Europe and America since the $19^{\text {th }}$ century. It originates from Korea and China, where it was known at least 2000 years ago. In addition to its undeniable decorative significance, the bleeding heart has a great potential in medicine and the cosmetics industry. This is due to the sap of the species containing numerous alkaloids and isoquinolines that have anti-cancer and anti-aging properties. The increasing number of home-grown bleeding heart cultivars, its considerable therapeutic properties, as well as the relatively small number of endemic populations known within its historic range, make it necessary to develop long-term storage methods of the species genetic resources. Among various conservation strategies, cryopreservation (storage in liquid nitrogen at $-196^{\circ} \mathrm{C}$ ) is believed to be the most efficient one, although the impact of severe stress, related to pretreatment and cooling /rewarming cycles, on the biological material stability is still ambiguous. There have been no reports on L. spectabilis cryopreservation. The aim of this pioneer study was to analyze the stability of the cryopreservation-recovered plant material on the molecular, biochemical and phenotypic levels.

In vitro-grown single node explants of $L$. spectabilis "Gold Heart" were precultured on Murashige and Skoog medium with $9 \%$ sucrose, $65 \mu \mathrm{M}$ kinetin and $10 \mu \mathrm{M}$ abscisic acid for seven days. Next, shoot tips, 1-3 mm in length, were dissected and encapsulated in $3 \%$ calcium alginate $(30 \mathrm{~min}$ ) and then osmoprotected in the Loading Solution (2.0 M glycerol and 0.4 M sucrose) for 20 minutes. Next, the explants were dehydrated with Plant Vitrification Solution 3 (PVS3; 50\% glycerol and 50\% sucrose, w/v) for 0; 30; 60; 90; 120; 150 or $180 \mathrm{~min}$. The dehydrated explants were placed in 2.0-ml cryovials and plunged in liquid nitrogen $\left(-196^{\circ} \mathrm{C}\right)$. After at least one hour, the cryovials were thawed in water bath $\left(39 \pm 1^{\circ} \mathrm{C}\right)$ for $3 \mathrm{~min}$. The explants were rinsed with Washing Solution (liquid MS medium with $1.2 \mathrm{M}$ sucrose) for $30 \mathrm{~min}$. Next, the beads were inoculated on the MS recovery medium with $3 \%$ sucrose and $2.32 \mu \mathrm{M}$ 6-benzyladenine for two months.

Plantlets recovered from the cryopreserved shoot tips were subjected to molecular analyses with the use of RAPD, ISSR and SCoT markers (six primers each). Moreover, the concentration of anthocyanins and chlorophylls (a, b and total) in the plant body, as well as the fresh weight, length and number of shoots and roots were determined. Results were referred to a positive (untreated but LN-stored) and negative (untreated) control.

The highest survival and recovery rates $(76.7 \%$ and $72.5 \%$, respectively) were recorded after 150 -min dehydration. No recovery was reported with the positive control, nor as at 0 and 30 min PVS3 treatment. The untreated (negative) control and shoot tips pretreated for 120 min produced the greatest number of shoots. Shoots recovered after 120 and 150-min dehydration were longer and more massive than the control, and regenerated more roots. On the other hand, the longest PVS-treatment reduced the length of the regenerated roots.

Biochemical analyses showed that dehydration with PVS3 for 180 min would lead to a significant anthocyanins and total chlorophyll content decrease in the recovered microshoots as compared with the control and other cryotreatments. Those results were confirmed by the phenotypic observation of those microshoots which lacked the purple coloration of the stems, typical for the "Gold Heart" cultivar. Similarly, the concentration of chlorophyll $b$ decreased in all cryotreated samples, although the level of chlorophyll a remained unaltered. On the other hand, no genetic variation in cryopreserved samples was detected by RAPD, ISSR and SCoT markers. 


\title{
Iridoid and phenylethanoid glycosides accumulation in in vitro cultures of plants from Rehmannia genus
}

\author{
EwElina PiąTCZaK \\ Department of Biology and Pharmaceutical Botany, Medical University of Lodz, Łódź, Poland \\ * ewelina.piatczak@umed.lodz.pl
}

Plants from Rehmannia genus belong to Orobanchaceae family [1]. It is a small genus consisting of 6 species naturally occurring in some provinces of China. Additionally, they are cultivated on plantations in Korea and Japan [2]. The member of the genus - $R$. glutinosa is a valuable medicinal plant species listed in The Chinese Pharmacopoeia [3]. Roots of the species, called Rehmanniae Radix, are widely used as fresh, dry or steamed roots as antianemic, antipyretic, antiinflammatory, hypoglycemic and antihypertensive agents [4]. Secondary metabolites presented in the roots, mainly iridoid glycosides (catalpol, aucubin, catalposide, harpagide, harpagoside), phenylethanoid glycosides (acteoside, isoacteoside) and polysaccharides (rehmannosides) are responsible for the properties of the roots. The aims of the presented studies were:

1) establishment of untransformed and transformed cultures (adventitious and axillary shoot cultures, hairy root cultures) and the whole regenerated untransformed and transformed plants of $R$. glutinosa and $R$. elata;

2 ) identification and quantitative analyzes of selected iridoid and phenylethanoid glycosides in plant material obtained in vitro and comparison of secondary metabolites with the content of the compounds in leaves and roots of seed-derived plants;

3) an increase in the metabolite production in hairy root culture by elicitation with methyl jasmonate and salicylic acid.

The most important achievement of the investigations was the detection of two pharmacologically important iridoid glycosides (harpagide and harpagoside) in plant material derived from in vitro cultures and from seed-derived plants of $R$. glutinosa and $R$. elata. The two iridoids have been not reported earlier in the genus Rehmannia. The highest harpagide contents were detected in the shoot culture and hairy roots of $R$. elata as well as in the whole regenerated transformed and untransformed $R$. glutinosa plants. The highest levels of catalposide, acteoside and isoacteoside were detected in hairy root cultures of $R$. glutinosa after elicitation with MeJA. Another achievement was the regeneration of $R$. glutinosa transformed plants from hairy roots with higher biomass and metabolite contents per plant than that of untransformed plants. Moreover, the untransformed and transformed shoot cultures of the s,pecies were successfully multiplied in the nutrient sprinkle bioreactor and as a result, the multiplication rates of the cultures were 1.6-2 times higher than the rates of the same shoot cultures in glass jars or tubes.

\section{References}

[1] The Angiosperm Phylogeny Group (2016) Bot. J. Linnean Soc. 181(1): 1-20. doi: 10.1111/boj.12385.

[2] Albach D.C., Li H.-Q., Zhao N., Jensen S.R. (2007) Biochem. Syst. Ecol. 35: 293-300. doi: 10.1016/j.bse.2006.11.003.

[3] Pharmacopoeia Commission of the People's Republic of China (2015) The pharmacopoeia of the People's Republic of China. Vol. 1. Chemical Industry Publishing House, Beijing.

[4] Kitagawa I., Fukuda Y., Taniyama T., Yoshikawa M. (1991) Chem. Pharm. Bull. 39: 1171-1176. 


\title{
A simultaneous root immobilization and in situ extraction for the intensification of secondary metabolism in Rindera graeca in vitro cultures
}

\author{
Katarzyna MaŃKo ${ }^{1}$, MATEUSz KaWKa ${ }^{2 *}$, Katarzyna SYKŁOWSKa-BARANEK ${ }^{2}$, \\ Maciej PilareK ${ }^{1}$, Agnieszka Pietrosiuk ${ }^{2}$ \\ ${ }^{1}$ Faculty of Chemical and Process Engineering, Warsaw University of Technology, Warszawa, Poland \\ ${ }^{2}$ Faculty of Pharmacy with Division of Laboratory Medicine, Medical University of Warsaw, Warszawa, Poland \\ *k.manko@outlook.com; *mat.kawka@gmail.com
}

The specificity of plant in vitro cultures allows to enhance the unique plant biosynthetic potential for the production of pharmaceutically valuable compounds [1] by various biotechnological techniques. In the presented work we examined the effects of biomass immobilization on polyurethane foam (PUF) applied simultaneously with an in situ extraction using perfluorodecalin (PFD) on the secondary metabolism alteration in Rindera graeca root cultures. An in situ extraction is a process of a secondary metabolites accumulation in the immiscible liquid phase (i.e. extractant or solvent) added into the culture system. The selection of PFD to the role of a novel in situ extractant was dictated mainly by its nontoxicity and other functionality e.g. as a liquid gas carrier due to the great solubility of gases [2]. The immobilization of roots provides a favorable microenvironment for producing plant secondary metabolites and enhances the resistance of the biomass from the hydrodynamic-based shear forces in a culture system [3]. R. graeca (Boraginaceae) is an endemic plant occurring in the mountain territories of Greece. The recent investigations on its phytochemical profile have demonstrated the presence of significant amounts of phenolic compounds and newly identified naphthoquinone which proved to be a promising anticancer agent [4]. In the current study four $R$. graeca root lines of different origin were cultured in vitro in a liquid DCR medium [5] for 42 days in non-immobilized and immobilized culture variants. The PFD supplementation of both types of cultures was performed on day 14. Independently, the effect of PFD-based aeration was investigated in root cultures. Samples were harvested on 28,35 and 42 day of culture. The content of the newly identified naphthoquinone (50,6-(isohex-1-en-1,2-diyl)-2-methoxynaphthazarin) (NQ) in extracts collected from root biomass, post-culture medium, PFD phase and PUF was quantified using the HPLC-DAD-UV-Vis method. The content of phenolic compounds in root extracts and their antioxidant activity was analyzed using the Folin-Ciocatleu's and DPPH radical scavenging methods, respectively. A simultaneous application of the PUF and PFD-based extraction resulted in a most significant production of $\mathrm{NQ}$ in the cultures of all examined root lines and its highest content was noted in the case of a callus-regenerated root line (RgKZNOA) $(1.181 \mathrm{mg} / \mathrm{flask})$. At the same time PUF immobilization without PFD-based extraction also resulted in high NQ contents, especially for RgKZNOA root line $(0.810 \mathrm{mg} /$ flask). The highest amount of phenolic compounds was observed in cultures of anatomical roots after supplementation with aerated PFD (1.682 mg GA/gD.W., i.e. 2.1-fold increase when compared to untreated control cultures). A similar tendency was observed for all examined root lines and the results corresponded with the antioxidant activity of extracts. Considering the significantly lower growth rates observed after the application of PUF or aerated PFD, both methods lead to a stress-induced secondary metabolism intensification. In all examined culture systems the application of PUF and PFD created an advantageous in vitro environment for the secondary metabolites accumulation in $R$. graeca root cultures, however with a clear dominance of the first method. The results of the presented studies on PUF and PFD-based in situ extraction system can be recognized as the successful practical approach for an enhanced production and a simplified isolation procedure of secondary metabolites in systems of in vitro plant biomass cultures.

\section{References}

[1] Kawka M. et al. (2017) Biul. Wydz. Farm. WUM 8: 68-79.

[2] Kawka M. et al. (2017) Biul. Wydz. Farm. WUM 7: 60-67.

[3] Mańko K. (2017) MSc. thesis.

[4] Jeziorek M. et al. (2012) Planta Med. PI371.

[5] Gupta P.K., Durzan D.J. (1985) Plant Cell Rep. 4: 177-179. 


\title{
Application of in vitro culture techniques in the maintenance of Linnaea borealis L. - protected species of Polish flora
}

\author{
BARBARA THIEM \\ Department of Pharmaceutical Botany and Plant Biotechnology, Poznan University of Medical Sciences, Poznań, Poland \\ *bthiem@ump.edu.pl
}

Twinflower (Linnaea borealis L.) is a plant species which belongs to Linnaeaceae family (previously Caprifoliaceae). It is found throughout the northern hemisphere in the circumboreal habitats. $L$. borealis commonly grows in tundra and taiga. In some countries in Europe, including Poland, Twinflower is under protection as a glacial relic $[1,3]$. It is a small, creeping, perennial plant, an evergreen, stoloniferous dwarf shrub characterized by the aboveground runners. The plant has an unique inflorescence which is composed of two bell-shaped, white or pinkish-white flowers growing on a stem in the shape of the letter "Y". L. borealis can be propagated mainly by vegetative and rarely generative methods [4].

The objective of this study was to develop an in vitro culture method for the preservation of $L$. borealis. The introduction of the plant to the in vitro conditions was time-consuming and very problematic. An in vitro culture was initiated using the stems pieces (with apical or lateral buds) of the plant from the natural sites (Woliński Park Narodowy) after receiving the special permission. The shoot cultures were multiplied on MS medium with BAP $\left(1.0 \mathrm{mg} / \mathrm{dm}^{3}\right)$ and $\mathrm{GA}_{3}\left(1.0 \mathrm{mg} / \mathrm{dm}^{3}\right)$. In the preliminary studies, $5-7$ new shoots/explant were observed. The leafderived callus was obtained on MS medium supplemented with picloram $\left(1.0 \mathrm{mg} / \mathrm{dm}^{3}\right)$ or $2,4-\mathrm{D}\left(2.0 \mathrm{mg} / \mathrm{dm}^{3}\right)$ with NAA $\left(0.2 \mathrm{mg} / \mathrm{dm}^{3}\right)$. Next, the procedure of somatic seeds production and short- and medium term storage was evaluated.

Our studies on the application of an in vitro technology for the protection of this endangered plant are also concentrated on its in vitro mass scale propagation. This is because of the ability of $L$. borealis to produce biomass with the ability to synthesize pharmacologically active secondary metabolites.

Plant biotechnology enables phytochemical studies of rare and protected species without depleting their natural sites. The aim of further investigations will be to find the best condition for L. borealis in vitro culture and to search for bioactive secondary metabolites in the obtained plant material.

\section{References}

[1] Alm T. (2006) Bot. J. Linnean Soc. 151: 437-452.

[2] Mikuła A., Makowski D., Tomiczak K., Rybczyński J.J. (2013) Polish J. Agron. 14: 3-17.

[3] Piękoś-Mirkowa H., Mirek Z. (2003) Flora Polski Atlas roślin chronionych. Multico Oficyna Wyd. Warszawa: ISBN 83-7073256-9.

[4] Thiem B., Buk-Berge E. (2017) Herba Polon. 63(3): 56-64. 


\title{
Biologically active terpenoid constituents from shoot cultures of Carpesium divaricatum
}

\author{
AnNa StoJaKowska*, Natalia KŁeczek, Janusz MalarZ \\ Department of Phytochemistry, Institute of Pharmacology, Polish Academy of Sciences, Kraków, Poland \\ *stoja@if-pan.krakow.pl
}

Carpesium divaricatum Sieb. \& Zucc. (Asteraceae, tribe Inuleae), a perennial herb distributed mainly in the Far East (China, Japan, Korea) has been used in the traditional medicine as a remedy against fever, inflammation, pain and parasitic diseases [1]. Chemical constituents of the aerial parts of the plant have been repeatedly studied by different research teams. The studies revealed the presence of numerous sesquiterpene lactones, mainly germacranolides, which were active in vitro against a number of human cancer cell lines. Moreover, shoots of the plant accumulated acyclic diterpenes and monoterpenoid thymol derivatives of moderate antimicrobial activity [1].

A shoot culture of $C$. divaricatum was obtained from aseptic seedlings. The seeds of the plant, delivered by the Research Center for Medicinal Plant Resources, National Institute of Biomedical Innovation in Tsukuba (Japan), were aseptically germinated on a hormone free, modified MS medium (macronutrients content reduced by 50\%), solidified with $0.8 \%$ agar. Cotyledonary nodes of the seedlings were used as primary explants. The explants were inoculated on MS medium containing benzylaminopurine (BA, $4.44 \mu \mathrm{M}$ ), napthaleneacetic acid (NAA, $0.54 \mu \mathrm{M}$ ) and $3 \%$ sucrose. The cultures were maintained at $25^{\circ} \mathrm{C}$, under continuous light $\left(40 \mu \mathrm{mol} / \mathrm{m}^{2} \mathrm{~s}\right.$, cool white fluorescent tubes). After an eight-week culture, secondary explants (nodal explants) were excised from the proliferated shoots and transferred either onto the medium of the same composition or onto the medium with a diminished content of growth regulators $(2.0 \mu \mathrm{M}$ BA and $0.1 \mu \mathrm{M} \mathrm{NAA})$.

Shoots of $C$. divaricatum, multiplied on a medium containing $2.0 \mu \mathrm{M}$ BA and $0.1 \mu \mathrm{M}$ NAA, were air-dried at room temperature, pulverized, and subjected to the RP-HPLC/DAD analysis to assess their capability to accumulate biologically active terpenoids. Standard compounds - thymol derivatives and some germacrane type sesquiterpene lactones (divaricins) - were previously isolated in our laboratory from shoots of field grown $C$. divaricatum plants and root cultures of Inula spp. The identities of the isolated compounds were confirmed by a spectral analysis (UV/Vis, ${ }^{1} \mathrm{H}$ NMR $400.17 \mathrm{MHz}$, HRESIMS). Chromatographic analysis of extracts from in vitro grown shoots of $C$. divaricatum confirmed the presence of sesquiterpene lactones and monoterpenoids, including 8,9-epoxy-10isobutyryloxythymol isobutyrate (1) and cardivin A (2). Compound $\mathbf{1}$, formerly found in root cultures of Inula spp. and a multiple shoot culture of Telekia speciosa was moderately active against Staphylococcus aureus and Candida albicans [2, 3]. Cardivin A demonstrated cytotoxic activity against human cancer cell lines in vitro $\left(\mathrm{ED}_{50} 1.16-3.19 \mathrm{mg} / \mathrm{ml}\right.$; doxorubicin $\left.-\mathrm{ED}_{50} 0.11-2.40\right) \mathrm{mg} / \mathrm{ml}$ [ [4].

\section{References}

[1] Zhang J.-P., Wang G.-W., Tian X.-H. et al. (2015) J. Ethnopharmacol. 163: 173-191.

[2] Stojakowska A., Kędzia B., Kisiel W. (2005) Fitoterapia 76: 687-690.

[3] Stojakowska A., Malarz J., Kisiel W. (2011) Acta Soc. Bot. Pol. 80: 253-256.

[4] Kim D.K., Baek N.I., Choi S.U., Lee C.O., Lee K.R., Zee O.P. (1997) J. Nat. Prod. 60: 1199-1202. 


\title{
Induced totipotent stem cells and somatic embryogenesis in plants: new answers to old questions
}

\author{
Marcin OlsZaK $^{1 *}$ Petr Berko $^{2}$, WoJciech BURza ${ }^{2}$ \\ ${ }^{1}$ Polish Academy of Sciences Botanical Garden - Center for Biological Diversity Conservation in Powsin, Warszawa, Poland \\ ${ }^{2}$ Department of Plant Genetics, Breeding and Biotechnology, Warsaw University of Life Sciences, Warszawa, Poland
}

The phenomenon of somatic embryogenesis (SE) is the object of a wide range of extensive research since years proceed for many plant species. Nevertheless, little is known about the mechanisms leading to the transition of the somatic cells into a totipotent embryogenic cell that can either be developed into an embryo. Our research is an proposal to look at the phenomenon of inducing SE in the context of intensively in recent years expanded knowledge about stem cells (SCs) in general - not only on plant organisms, but inspiration were especially animal and human induced pluripotent SCs. The current definition of stem cells is that they are clonogenic precursors whose daughters can either remain stem cells or undergo differentiation and the single cells can be genetically marked and their descendants identified by the spread of this label [1]. Classically, the term pluripotent SCs is used in plants for cells located within a niche in meristems. More than decade ago Verdeil et al. (2007) proposed to extend the plant SC concept to include single embryogenic cells as a totipotent SC based on their capacity to regenerate or develop into an embryo under certain conditions. Following this trail and looking for inspiration in the reports from the field of animal kingdom research, we analyzed the process of acquisition of totipotency by somatic cells in cucumber (Cucumis sativus L.), which has an interesting course in which constitutively synthesized riboflavin (RF) derivative is involved. Importantly, induced totipotent SCs, despite dedifferentiation, retain the ability of the initial explants (e. g cotyledon) to synthesize and accumulate RF. Under suspension conditions, these cells form pro-embryogenic mass (PEM) aggregates. Importantly, embryo cells are differentiating from single PEM cell through asymmetric division do not show this feature. Cucumber PEM cells meets the criteria of the above definition of SCs - they can proliferate, undergo differentiation or death. Such cell are also labeled by RF source green autofluorescence and have a number of unique features, such as the ability to self-sustaining proliferation (and induction) on media without growth regulators, a constantly occurring de novo re-initiation process of subsequent generations through the still-unclear process of reprogramming the somatic cell to the SCs state. Interestingly, similar characteristics have an animal SCs, also the conditions and the way they are induced, in principle, can be similar. Numerous reports from animal and human area pointing to the important role of epigenetic changes in this process prompted us to make the far-reaching hypothesis that plant SCs (being progenitors for somatic embryos) arise as a result of epigenetic reprogramming through endogenous factors, produced and secreted into the environment by previous induced SCs. In humans are known examples of the paracrine effect of SCs on differentiated cells, through the secretion of substances such as cytokines or even RNA molecules and lipids. It happens in wound healing and cancer progression [3]. These results clearly indicate that SCs and its differentiated daughter cells may influence their condition using very precise (but still unclear) interactions. Our results show a similar mechanism and thus confirm the thesis put forward by Jiang et al. (2014) that the influence of plant SCs plays a key role in the process of dedifferentiation. An attempt to look for similarities in the process of induction of SE in a basis as analogous to the induction of totipotent SCs may shed a new light on the problem of understanding the mechanisms underlying this essential for plant biotechnology process.

\section{References}

[1] Laux T. (2003) Cell 113: 281-283.

[2] Verdeil et al. (2007) Trends Plant Sci. 12: 245-252.

[3] Dittmer J., Leyh B. (2014) Int. J. Oncol. 44(6): 1789-1798.

[4] Jiang et al. (2014) Frontiers Plant Sci. 6: 1028-1028. 


\title{
Biotechnological methods used in Brassica napus resynthesis
}

\author{
LaurencJa Szata*, Katarzyna Sosnowska, Teresa Cegielska-Taras \\ Plant Breeding and Acclimatization Institute - National Research Institute, Poznań Division, Poznań, Poland \\ *1szala@nico.ihar.poznan.pl
}

Oilseed rape (Brassica napus L.) is considered as relatively young species. It was formed through of multiple spontaneous and independent natural hybridizations between $B$. rapa and $B$. oleracea. The short domestication period, a lack of direct wild ancestors and intensive plant breeding activities, especially for double zero quality, have resulted in a narrow genetic diversity of this species. Whereas B. rapa and B. oleracea are both highly polymorphic and offer a much broader genetic variability that can be exploited for oilseed rape improvement via experimental hybridization - resynthesis - from diploid progenitors. Resythesized B. napus (RS) can be used to increase the genetic diversity and to develop a heterotic genepool that is a requirement for successful hybrid breeding programmes.

Interspecific breeding is considered to be the most important source of genetic variation. The introgression of genes may be part of a breeding programme towards disease resistance, better growth vigor, winter hardiness or morphological alteration in general. Interspecific crosses within the genus Brassica were carried out already in the 1920 s and 1930s in Japan. However the production of interspecific hybrids was difficult due to two major hybridization barriers that impede the gene flow between species: prezygotic (before-fertilization) and postzygotic (afterfertilization) barriers. The development of new biotechnologies in the in vitro culture allowed effective interspecific, both sexual (pollination) and asexual (protoplast fusion), hybridization.

In the sexual hybridization between distant species, incompatibility barriers (pre-and postzygotic obstacles) often prevent interspecific crosses. In prezygotic barriers, pollen tubes may start to grow, but then they do not reach the ovules due to the presence of incompatibility mechanisms operating in the stylar tissue. Direct in vitro pollination of stigma or pistils and opened ovaries or ovules may be useful in overcoming the incompatibility barriers. In such cases the style or part of it can be excised and pollen grains either placed on the cut surface of ovary or transferred through a hole in the wall of ovary. Whereas postzygotic barriers can be avoided by an in vitro culture of enlarged ovules with embryos. They are isolated when they are still at an early stage of development and are grown on a medium replacing properly formed endosperm (embryo rescue).

Protoplast fusion may offer several potential advantages over sexual crosses in hybrid production, including novel cytoplasmic combinations and retention of parental heterozygosity, hybridization of a sterile plant that may have desirable attributes, opportunity for potential chromosomal associations or modification of chromosomal constitutions while in vitro, and a possibility of maintaining desirable gene combinations, especially if heterozygous and quantitative. However, experience with somatic hybrids shows that plant regeneration after protoplasts fusion is slow and the regeneration efficiency is low.

In our Laboratory we performed $B$. napus resynthesis by crossing three subspecies of $B$. rapa and two subspecies of $B$. oleracea using biotechnological methods: in vitro pollination and embryo rescue (Sosnowska and CegielskaTaras, 2014). To improve the quality of the seeds of resynthesized oilseed rape it was crossed with double low quality natural lines and then from F1 hybrids DH semi-resynthesized lines were developed using isolated microspore culture (Szała et al., 2016). Nowadays in vitro methods are widely applied in the production of novel genotypes because they are convenient methods and have a potential to reduce the breeding cycle of oilseed rape and help create new cultivars.

Project No. 51 of the Ministry of Agriculture and Rural Development of Poland. 


\title{
In vitro culture and biotechnology of Lycopodiaceae sensu lato. Have hopes been fulfilled?
}

\author{
Wojciech Szypula*, Agnieszka Pietrosiuk \\ Department of Pharmaceutical Biology and Medicinal Plant Biotechnology, Medical University of Warsaw, Warszawa, Poland \\ *wszypula@wum.edu.pl
}

The methods of in vitro culture of plant cells, tissues and organs were examined and given much attention at the beginning of the last century, which resulted in the setting the first commercial laboratories over 60 years ago [1]. Unfortunately, efforts in this area have achieved only limited success. This problem concerns mainly club mosses. Club mosses are a compact group of microphyllus plants and have relatively small diversity [2]. Today they constitute less than $1 \%$ of modern flora [2]. Although club mosses are very diverse and virtually cosmopolitan, the family Lycopodiaceae s.1. is considered as monophyletic [2]. In vitro techniques have been rarely used in different Lycopodiaceae s.l. in recent decades. In the case of some species from Lycopodiaceae s.l., the great morphogenetic potential and plasticity of in vitro cultures is visible [3-15]. Organogenesis or embryogenesis were achieved on some gametophytic or sporophytic explants (shoots, juvenile gametophytes, propagule, somatic embryo) with or without growth regulators in the media [3-15]. Such systems are an excellent tool for studying the factors affecting morphogenesis and embryogenesis and thanks to their use, new information has been obtained about the mechanisms involved in these processes was obtained [4-14]. Doubtless, such systems are also a promising tool for studying developmental, biochemical and physiological processes in club mosses. Up to date several regeneration systems of club mosses applicable to mass propagation have been described. In the 1950s Freeberg Wetmore [5] and Freeberg [6] described gametophyte cultures and apogamous development of sporelings of some club mosses. Other partially successful cultures involved gametophytes of various species of Lycopodium [7-9]. Recently, Atmane et al. [10] and Bienaime et al. [11] maintained callus culture and indirect somatic embryogenesis of Lycopodiella inundata. Furthermore Szypuła et al. [3, 12] described shoot cultures, sporophytes propagations from bulbil and somatic embryogenesis of Huperzia selago and regardless of Whittier and Strochova [13] reported in vitro cultures of gametophytes $H$. selago [14]. Similarly tissue cultures of $H$. pinifolia [4] and Phlegmariurus squarrosus [15] have been achieved.

\section{References}

[1] Malepszy A. (2010) Biotechnologia 2(89): 10-17.

[2] Szypuła W. (2013) Acta Bot. Siles. 9: 25-56.

[3] Szypuła W. et al. (2013) Acta Soc. Bot. Pol. 82: 313-320.

[4] Ishiuchi K. et al. (2013) Phytochemistry 91: 208-219.

[5] Freeberg J.A., Wetmore R.H. (1957) Phytomorphol. 7: 204-217.

[6] Freeberg J.A. (1957) Phytomorphol. 7: 217-229.

[7] Whittier D.P. (1977) Can. J. Bot. 55(5): 563-567.

[8] Whittier D.P. (1981) Bot. Gaz. 142: 519-524.

[9] Whittier D.P., Webster T.R. (1986) Am. Fern. J. 76(2): 48-55.

[10] Atmane N. et al. [2000] Plant Sci. 156(2): 159-167.

[11] Bienaimé C. et al. [2015] Plant Cell Tiss. Organ. Cult. 123: 523-533.

[12] Szypuła W. et al. [2005] Plant Sci. 168: 1443-1452.

[13] Whittier D.P., Storchova H. [2007] Am. Fern. J. 97(3): 149-154.

[14] Szypuła W.J. [In:] Proceedings of the 56th meeting of the Polish Botanical Society. Polish Bot. Soc. Olsztyn 2013: $167-168$.

[15] Ma X., Gang D.R. [2008] Phytochemistry 69(10): 2022-2028. 


\title{
DNA barcoding and banking as a way to preserve forest biodiversity in the Białowieża Forest, Poland
}

\author{
Ewa KaczmareK *, Matgorzata Palucka \\ Forest Gene Bank in Kostrzyca, Miłków, Poland \\ *Ewa.Kaczmarek@lbg.lasy.gov.pl
}

According to the International Union for Conversation of Nature, there are 24230 endangered plant species globally and their number still rises. Polish forest ecosystems are no exception. The Kostrzyca Forest Gene Bank (FGB) project Molecular identification (DNA barcoding) and biobanking of selected plant species from the Białowieża Forest, directly addresses this threat by prioritizing collecting and storing forest genetic resources. This includes seed material and DNA samples collected from the Białowieża Forest (BF). Project is financed by the Forest Fund of the State Forests National Forest Holding. Since the project's beginning in 2017, the Kostrzvca FGB has already gathered seed material of 18 plant species and DNA samples of 87 plant species. The overall goal of the project is to collect plant material of 112 species, representing 53 genera and 26 families within the BF flora. Thus, genetic collection from the $\mathrm{BF}$ is ideal due to its high biodiversity that also includes some of the most vulnerable and endangered plant populations (e.g. Arnica montana L., Viola epipsila LEDEB., Carex buxbaumii WAHLENB.). Cryogenic storage of seed samples $\left(-196^{\circ} \mathrm{C}\right)$ of these vulnerable plant populations ensures future reintroduction possibilities. This process can help maintain and encourage biodiversity which ultimately determines an ecosystem's survival.

The Kostrzyca FGB extends its forestry conservation efforts by creating DNA bank in DNA Analysis Laboratory. The DNA bank enhances the Kostrzyca FGB's conservation efforts by preserving ex situ the gene pool of the BF plant species. Currently, the DNA Analysis Laboratory stores 1256 tissue samples, 492 DNA samples and 175 associated voucher specimens. Herbarium collection is also utilized to provide additional resources in future botanical research and genetic analysis.

Lastly, the DNA Analysis Laboratory of the Kostrzyca FGB applies a DNA barcoding system for all specimens collected to improve taxonomic identification of plant species. Barcoding technology enables more efficient data management, conservation efforts, communication with other DNA banks and discrimination of cryptic species. Currently, DNA barcoding process requires the following steps: 1) field plant collecting, 2) tissue sample storage, 3) tissue subsampling for DNA extraction, 4) tissue disruption, 5) DNA extraction for non-kit and kit-based protocols, 6) PCR amplification of $m a t \mathrm{~K}, r b c \mathrm{~L}$, trn $\mathrm{H}-p s b \mathrm{~A}$, internal transcribed spacers, 7) PCR product determination, 8) cycle sequencing, 9) cycle sequencing reaction cleanup and processing for an ABI genetic analyzer, 10) sequence analysis, 11) matching the barcode sequence of unknown sample against the barcode library (BOLD, NCBI) for genetic identification.

Key words: Białowieża Forest, DNA bank, DNA barcode, genetic resources 


\title{
Single- versus multiple-cell origin of somatic embryos in the tree fern Cyathea delgadii
}

\author{
Matgorzata Grzyb *, Jan Jarostaw Rybczyński, AnNa MikuŁa \\ Polish Academy of Sciences Botanical Garden - Center for Biological Diversity Conservation in Powsin, Warszawa, Poland \\ *m.grzyb@obpan.pl
}

Cyathea delgadii is the first species belonging to monilophytes for which the propagation by somatic embryogenesis (SE) has been described [2]. In this species, the somatic embryo formation is induced independently of plant growth regulators, and its efficiency is regulated by a number of chemical and physical factors e.g. light, sucrose, concentration of micro- and macroelements of the MS medium [1]. Moreover, the embryogenic capacity can be affected by some explant features, such as, for instance, its type and length, or the physiological state of donor plants. In this context, the objective of this study was to investigate the possible role of the explant type and length and diameter of the explant-donor frond on both the pathway of somatic embryo differentiation and efficiency of SE in C. delgadii.

The research was based on the system of SE developed in our laboratory for the tree fern $C$. delgadii [1, 2]. Initial explants (i.e. stipes and internodes) were collected from the youngest sporophyte frond. The analyses were performed at various stages of culture conducted on a hormone-free medium containing half-strength Murashige and Skoog's macro- and micro-nutrients and full complement of vitamins (1/2MS) supplemented with $1 \%$ sucrose, in the dark. To visualize the structural and morphological changes involved in the primary explants dedifferentiation and somatic embryo formation the light and environmental scanning electron (ESEM) microscopy was used. For light microscopy, stipe and internode explants were fixed in a mixture of $2.5 \%$ paraformaldehyde and $2.5 \%$ glutaraldehyde, and embedded in Epon epoxy. ESEM studies were conducted on not fixed material.

The results show that the source of explants determines both the pathway of the somatic embryo formation and SE efficiency. We demonstrated for the first time the structural evidence that the fern somatic embryos may develop not only from single epidermal cells, but also from groups of rapidly dividing cells of epidermal and cortical layers. Somatic embryos derived from stipe explants followed a unicellular pathway, whereas those derived from internodes were multicellular in origin. The whole developmental sequence from the first embryogenic cell divisions of stipe and internode explants to fully differentiated embryo was documented. Although both explant types investigated were able to produce somatic embryos, the morphogenic capacity of internodes was much lower and was only two somatic embryos per responding explant. We showed that the efficiency of SE dependent on the length and diameter of the first frond used as the source of stipe explants, whereas the length of internode explants does not influence the somatic embryo formation. The highest efficiency of SE (92\%) was obtained on stipe explants that were excised from the first frond, measuring $10 \mathrm{~mm}$ in length and less than $300 \mu \mathrm{m}$ in diameter. The explants produced on average 21 somatic embryos.

Two pathways of somatic embryo differentiation described here may provide good models for studying the mechanism of SE induction associated with a single- and multiple-cell proliferation. Conducting research for both type of explants can significantly help to better understand the mechanisms underlying the acquisition of embryogenic competence by plant somatic cell.

\section{References}

[1] Mikuła A., Pożoga M., Grzyb M., Rybczyński J.J. (2015a) Plant Cell Tiss. Organ. Cult. 123: 467-478.

[2] Mikuła A., Pożoga M., Tomiczak K., Rybczyński J.J. (2015b) Plant Cell Rep. 34: 783-794. 


\title{
Various levels of the morphogenic phenomenon in tree ferns in in vitro culture
}

\author{
JAN J. RYBCZYŃSKI *, KaROLINA TOMICZAK, ANNA MIKUŁa \\ Polish Academy of Sciences Botanical Garden - Center for Biological Diversity Conservation in Powsin, Warszawa, Poland \\ *j.rybczynski@obpan.pl
}

The history of the progress of plant experimental biology has been indicated by working with species that were accepted as model plants because of their morphogenic potential. This potential was described on various levels of plant body organization: organs, tissues, cells and their organelles. The dominant role of seed plants in a human being's life found its expression in advanced progress of the analysis and exploration of genomes of these plants. And yet cryptogamic plants and in particular ferns may appear in the living structure, which recognition might use the tools of the seed plant genome methods. In the case of ferns, the biotechnological methods helped to discover a completely new phenomenon never described till now in the plant.

Considering the involvement of our studies in the biotechnology of tree ferns we would like to present our recent results and will try to support the opinion that ferns are excellent material to carry on studies of plant experimental biology using biotechnological tools. Since 2013 the way of fern gene manipulation has been opened. Transgenesis was for the first time demonstrated in Pteris vittata and Ceratopteris thalictroides with the help of $A$. thumefaciens when spores were chosen as targets and were later used at various stages of gametophyte development. Among two alternating generation, the sporophyte were chosen by us. And during the Conference the following subjects will be presented: in vivo and in vitro tree fern profile, organogenesis versus somatic embryogenesis, cytokinin effect on morphogenesis processes, green globular bodies production, liquid culture in vegetative propagation, characteristics of liquid cell suspensions and protoplast isolation and the attempts of their culture. Special attention will be paid to the culture conditions: light/darkness and plant growth hormones are crucial.

Papers published earlier which concerned the description of gametophyte and sporophyte cultures of tree ferns and their unlimited in vitro production constitute the background for the creation of new areas of in vitro experiments. These fern cultures allow exploration of the morphogenetic potential not only in predisposed structure like meristem but also in cell organized complexes and undifferentiated cell aggregates, single cells and their protoplasts. Cytokinins like TDZ or the combination of auxins and cytokinins are required for the induction of the organogenesis. This stays in contradiction to the hormone free conditions of the somatic embryogenesis. In our experiments a liquid culture created new conditions for an easier consumption of the medium ingredients by cultured explants and helped to modify their growth and development. In this type of culture, the meristem of tree ferns expressed various responses according to the volume of the medium and the size of tissue which were cultured in it. Very poor results and consequently a limited number of papers concerning protoplast culture of both fern generations drove us to establish a cell suspension that could be used as the source of protoplasts. The dynamically growing cell suspension aggregates supplied the experiment with very young material with not yet finally definite cell wall, which seemed to us to be the alternative object to gametophyte green cells and green leaf mesophyll cell with fully formed walls. In the case of a protoplast culture, the liquid medium does not quite often offer the best conditions for protoplast cell division, and the agarose embedding may appear to be the best medium for this purpose. This is a question of the future progress of the fern genome modification. 


\title{
Proteins interacting with $\gamma$-secretase subunits - on the way to elucidate functions of the proteolytic complex in Arabidopsis thaliana
}

\author{
Wojciech Kwiatkowski, Tomasz Skrzypczak, Przemyseaw WoJTaszeK * \\ Department of Molecular and Cellular Biology, Adam Mickiewicz University, Poznań, Poland \\ *przemow@amu.edu.pl
}

Gamma-secretase is a complex of four proteins, each with at least one transmembrane domain. In most cells, the complex includes: presenilin (PS1 or PS2), nicastrin (NCT), APH-1 (anterior pharynx-defective 1) and PEN-2 (presenilin enhancer 2) - all are present in 1:1:1:1 ratio. Over the years, presenilins and $\gamma$-secretase have been very extensively investigated in animals, particularly in humans, due to its participation in the regulation of the Notch signaling pathway, and in the processing of amyloid precursor protein (APP). Mutations in the $\gamma$-secretase complex may lead to abnormal cleavage of APP, and this is directly correlated with an etiology of Alzheimer's disease.

The roles of $\gamma$-secretase complex in plants are almost completely unknown. In $A$. thaliana protoplasts, the existing data confirm localization of $\gamma$-secretase subunit homologs within endomembrane system. Interestingly, however, none of the known major substrates of the animal $\gamma$-secretase complex, has been identified in plants. Presenilin and the whole complex of $\gamma$-secretase has been hypothetically considered as a complex regulating vesicular transport.

Here we show contribution of $\mathrm{Y}$-secretase in endocytosis in $A$. thaliana. Impact of PS1/PS2 on endocytosis was analyzed by observation of FM4-64 dye endocytosis in seedlings (WT and ps1/ps2 mutant plants). Interactions during endocytosis between $\gamma$-secretase complex and receptor proteins - FLS2 and BRI1 were also examined. Moreover, the capability of the complex to react with animal hTFR (human transferrin receptor) was analyzed. These proteins are not the direct substrates of the complex, but their correct endocytosis depends on PS1/PS2 activity. The obtained results suggest that the $\gamma$-secretase complex, contrary to the assumptions, does not affect the location of the tested receptors.

This research is supported by the National Science Centre grant No. 2012/05/B/NZ3/01026 to PW and KNOW RNA Research Centre in Poznań (No. 01/KNOW2/2014). 


\title{
Poster no. 2
}

\section{AUXIN RESPONSE FACTOR19 is involved in the shoot organogenesis induced in Arabidopsis thaliana explants cultured in vitro}

\author{
Barbara WóJcikowska, Matgorzata GaJ \\ Department of Genetics, Faculty of Biology and Environmental Protection, University of Silesia, Katowice, Poland \\ *barbara.wojcikowska@us.edu.pl
}

Members of the AUXIN RESPONSE FACTOR $(A R F)$ gene family play a key role in the regulation of the auxin response gene expression and control of the auxin-dependent developmental processes in plants including morphogenic responses induced in in vitro cultured explants. We found that the expression of numerous $A R F$ genes including ARF19 was significantly modulated during de novo shoot organogenesis (S-ORG) induced in Arabidopsis. This finding motivated us to study the role of $A R F 19$ in this process.

ARF19 binds to the AuxRE motif in the promoters of the auxin-responsive genes and in result the gene transcription is activated [2]. The function of ARF19 in control of the leaf expansion and lateral root growth during in vivo plant development was reported [1] but the involvement of this gene in shoot regeneration was not studied. To efficiently induce shoot regeneration in Arabidopsis two explant types were used: the cotyledons isolated from immature zygotic embryos and two weeks old seedling's roots. The explants were cultured for 7 days on an auxin medium inducing callus tissue (CIM) and then they were transferred onto the shoot induction medium (SIM) supplemented with cytokinin and auxin. The expression profile of ARF19 during S-ORG was evaluated and the significant up-regulation (over 3-fold) of the gene transcripts during callus and shoot formation on CIM and SIM media, respectively, was indicated. In support for the role of $A R F 19$ in S-ORG, the arf19 mutant was found to be impaired in capacity for the shoot regeneration and the average number of shoots regenerated per explant was significantly decreased in the mutant culture. The engagement of $A R F 19$ in shoot organogenesis was further confirmed in an analysis of the $p A R F 19-G F P$ reporter line. The inspection of the $p A R F 19-G F P$ explants during S-ORG indicated that $A R F 19$ expression was intensively activated in the early culture (3 and 5 day-old) induced on CIM medium and that result was consistent with the results of RT-qPCR analysis. The pattern of ARF19 expression was drastically changed in the explants transferred onto SIM medium, and the GFP signal became restricted to the explant areas regenerating shoots.

Altogether, the results provided some lines of evidence that $A R F 19$ positively controls shoot regeneration in Arabidopsis in vitro culture. Within the candidate targets of ARF19, the LOB DOMAIN-CONTAINING PROTEIN 16 and 29 genes encoding transcription factors controlling lateral root formation might be considered.

\section{References}

[1] Okushima Y., Fukaki H., Onoda M., Theologis A., Tasaka M. (2007) Plant Cell 19: 118-130.

[2] Weijers D., Wagner D. (2016) Ann. Rev. Plant Biol. 67: 539-574. 
Poster no. 3

\title{
Evaluation of the nuclear DNA content/ploidy level and some morphological traits of the rootstocks for cherry trees collected in the gene resources of the Research Institute of Horticulture in Skierniewice
}

\author{
MaŁgorzata Podwyszyńska*, MirosŁaw Sitarek, Agnieszka Marasek-CioŁakowsKa \\ Research Institute of Horticulture, Skierniewice, Poland \\ * Malgorzata.Podwyszynska@inhort.pl
}

Genome size is a fundamental parameter in many genetic and biological studies involving genome organisation, species relationships, and breeding works. Knowledge of the ploidy level can be useful in evaluating reproductive and somatic compatibility, an important parameter in scion and rootstock breeding programs. The evaluation of the nuclear DNA content was performed in order to determine the ploidy level of rootstocks of genus Prunus used in the growing of sour and sweet cherries. At the first stage of the research, the conditions of the flow cytometry analysis (with the application of propidium iodide for DNA staining) (FCM/PI) were optimized: the type of buffer for nuclei extraction and incubation time as well as the selection of internal standards (genotypes with a known nuclear DNA content). Repeatable results and relatively good-quality histograms were obtained using Partec extraction buffer with 1\% PVP addition, with incubation time longer than $50 \mathrm{~min}$. The best internal standard for the FCM analysis of cherry rootstocks with a nuclear DNA content from about 0.7 to $1.4 \mathrm{pg}$ proved to be Solanum lycopersicum $(2 \mathrm{C}=1.96 \mathrm{pg})$, whose peaks $2 \mathrm{C}$ and $4 \mathrm{C}$ did not coincide with the peaks of the genotypes tested, but overlapped partially with the peaks of Prunus genotypes with larger genomes tested. For rootstock genotypes with a nuclear DNA contents from 1.8 to $2.3 \mathrm{pg}$, Glycine $\max (2.91 \mathrm{pg})$ or Zea mays $(2 \mathrm{C}=5.44 \mathrm{pg}$ ) were selected as the internal standards. At the second stage, the optimized method and well-chosen internal standards allowed to assess with high precision the nuclear DNA content/ ploidy level of 20 Prunus genotypes of the rootstocks for cherries and sweet cherries. For the reference Prunus genotypes of the known chromosome number, 2C DNA values was $0.68 \mathrm{pg}$ for diploid $P$. cerasifera var. divaricata Led. "Anna", the value of 1.18 pg for triploid "GiSelA 3" (P. cerasus "Schattenmorelle" $\times P$. canescens $)$ and 2.16 pg for hexaploid $P$. domestica "Eruni". In nine rootstocks for sour cherry and sweet cherry trees ("F 12/1", "PiKu 1", "PiKu 3", "INRA SL 64", "Maxma Delbard 14 Brokforest”, "Ferci SL 405”, "GM 79", "LC-13", "L-2"), 2C DNA values ranged from 0.74 to $0.86 \mathrm{pg}$ indicating their diploid chromosome number. Nine rootstocks were identified as triploids ("Colt”, "GiSelA 3", "GiSelA 5", "GiSelA 6", "P-HL A", "P-HL C", "PiKu 4", "VSL 1" and "VSL 2") with their 2C DNA contents from 1.03 to $1.24 \mathrm{pg}$. Two rootstocks were considered to be tetraploids ("WC-13" and "LC 52") having $2 \mathrm{C}$ DNA of $1.39 \mathrm{pg}$. The ploidy level of the genotypes of sour cherry and sweet cherry rootstocks was evaluated in relation to the morphological and agronomical traits.

\section{Acknowledgement}

This work was performed in the frame of a multiannual programme on the preservation of the gene bank resources financed by the Polish Ministry of Agriculture and Rural Development: Task 1.3 "Collecting, preservation in ex situ collections, cryoconservation, evaluation, documentation and using of gene bank resources of horticultural crops". 


\title{
Poster no. 4
}

\section{Protoplast fusion in cabbage - characterization of culture and molecular evaluation of fusion products}

\author{
AgnieszKa KieŁKowska *, Marek SzklarczyK, Adela Adamus, Marta Solarz, \\ Marcelina WaJdziK, Beata Domnicz, AnNa Szlachtowska \\ Institute of Plant Biology and Biotechnology, Faculty of Biotechnology and Horticulture, \\ Agricultural University in Cracow, Kraków, Poland \\ *a.kielkowska@urk.edu.pl
}

Selected accessions of Brassica oleracea L. var. capitata, differing in ploidy level $(1 n, 2 n=2 x)$ were used as a plant material. Haploids were obtained by means of isolated microspore cultures. Diploid plants were obtained from seeds. Protoplasts were enzymatically isolated from leaves of in vitro grown plants according to the protocol of Kiełkowska and Adamus [2]. The purified protoplasts were suspended in $0.4 \mathrm{M}$ mannitol and, in order to discriminate fusion components, stained with fluorescent dyes (fluorescein diacetate (FDA) and rhodamine B). Electrofusion was performed using Eppendorf Multiporator cell fusion module equipped with a helix chamber. As controls we used cultures that had not been subjected to electrofusion. All cultures were immobilized in thin alginate layers [3] and cultured in the dark. The overall frequency of fusion was $8-20 \%$; however the frequency of heterokaryons, exhibiting double (red-green) fluorescence, was lower and was $2-12 \%$ depending on the parental combination. On the fifth day of culture, the viability of protoplasts subjected to electrofusion was slightly decreased (33-50\%) when compared to the control cultures (47-51\%). We observed differences in the mitotic activity of protoplast-derived cells depending on the ploidy of donors from which the protoplasts were isolated. On the fifteenth day of culture the highest (39\%) mitotic activity was observed in the control cultures of diploid accessions. The frequency of the mitosis of cells subjected to electrofusion was lower and ranged from $3 \%$ to $12 \%$ in haploid accessions and from $5 \%$ to $30 \%$ in diploid accessions. A sustained mitosis resulted in multi-cell colonies. After approximately 3 weeks of culture the alginate layers were overgrown with yellow-whitish colored calli. Callus formation was observed in all fusion combinations and in the controls. Regeneration of plants from the protoplast cultures in Brassica sp. is often a long and difficult process [1], therefore here, we performed molecular screening of the fusion products at the stage of callus. The first screen of the obtained population, with 10 selected nuclear and 8 organellar genome markers, revealed the hybrid nature of some calluses. More putative hybrid calluses were observed after the fusion of haploid accessions. Although within the examined pool the number of hybrids may be higher, their identification would require the use of additional markers.

\section{Acknowledgements}

This research was supported by the Polish Ministry of Agriculture and Rural Development (No. HOR.hn.802.24.2017).

\section{References}

[1] Holme I.B., Torp A.M., Hansen L.N., Andersen S.B. (2004) Theor. Appl. Genet. 108: 1513-1520.

[2] Kiełkowska A., Adamus A. (2012) In Vitro Cell Dev. Biol. 48: 265-273.

[3] Kiełkowska A., Adamus A. (2014) Acta Biol. Cracov Bot. 56(2): 20-26. 
Poster no. 5

\title{
Intergeneric crosses of selected legumes through the in vitro ovary culture
}

\author{
AURELIA ŚluSARKIEWICZ-JARZINA ${ }^{1 *}$, MARIA KATARZYNA WOJCIECHOWICZ ${ }^{2}$, \\ HaNNA PUdELSKa ${ }^{1}$, ANETTA KUCZYŃSKa ${ }^{1}$ \\ ${ }^{1}$ Institute of Plant Genetics Polish Academy of Sciences, Poznań, Poland \\ ${ }^{2}$ Institute of Experimental Biology, Faculty of Biology, Adam Mickiewicz University in Poznań, Poznań, Poland \\ *aslu@igr.poznan.pl
}

The aim of the study was to produce economically valuable lupine plants (resistant to anthracnose) and peas (with a stiff stem) through inter generic hybridization.

Species of the genera Lupinus and Pisum were selected as the maternal plants, while plants of the genera Lathyrus, Cicer, Lens and Vicia were used as donors of pollen. Plant materials for intergeneric crosses within the Lupinus species were L. albus and L. angustigolius (Wars and Tango). On the other hand, Pisum sativum (Acord and Audit) and Vicia faba v. Equina (VSAT 131), Vicia narbonensis (VNAR 2), Lens culinaris (LCU 131), Cicer arietinum (CAR 140), Lathyrus cicera (C27) were used for interspecies crosses. For each cross combination, about 300 pollinations were carried out. The isolated ovaries were placed on MS medium without growth substances, and were pollinated and cultured in the dark, at $24^{\circ} \mathrm{C}$ for 4 weeks.

Microscopic analyses of enlarged ovules from cultures were performed. Samples were fixed in a mixture of paraformaldehyde (2\%) and glutaraldehyde (2\%) and subjected to histological examination after paraffin embedment, and safranin and fast green staining. Moreover, the observations of pollen germination and pollen tube growth with aniline blue staining were done. The microscopic analysis of the prepared paraffin preparations was carried out using light microscopy. Otherwise, all crushed preparations were observed in an epifluorescence microscope.

In the study, pollen germination and pollen tube growth were observed in crosses between peas and grass pea. Pollen tubes were grown through ovaries and they entered the micropyle of ovules 6 days after culture. After 5 days of cultures, mature embryo sacs with egg cell, synergids and antipodal cells were present on the histological sections of the ovules. Some of the embryo sacs were shrunken and showed signs of degeneration. However, most of the embryo sacs remained alive in cultures and they contained egg cell with dense cytoplasm, prominent nucleus and nucleolus. A histological analysis showed that 2-6 celled proembryos were formed in the cultures.

The enlarged ovules and calli that were subsequently obtained were cultivated on different media at $22^{\circ} \mathrm{C}$ and light density of $80-100 \mu \mathrm{mol}$ photons $/ \mathrm{m}^{2} \mathrm{~s}$ for 12 hours. The callus tissue obtained in crosses was transferred on various media supplemented with BAP and NAA several times in different concentrations. All tested media promoted callus proliferation. These studies are a valuable source of information and will be continued as a part of the project, task 2.4. Distant crosses within the Lupinus, Pisum and Vicia - searching for new genetic variability and a way to shorten the breeding cycle using in vitro cultures.

\section{Acknowledgements}

The studies were supported by the Ministry of Agriculture and Rural Development, the National Multi-Year Project (2016-2020), Resolution no. 222/2015, 15 December 2015. Task 2.4. Distant crosses within the Lupinus, Pisum and Vicia - searching for new genetic variability and a way to shorten the breeding cycle using in vitro cultures. 


\title{
Poster no. 6
}

\section{Comparison of methods for obtaining virus free garlic (Allium sativum L.) propagated in in vitro cultures}

\author{
Waldemar Kiszczak, Urszula Kowalska, Tadeusz Malinowski, Krystyna GóRecka \\ Research Institute of Horticulture, Skierniewice, Poland \\ *waldemar.kiszczak@inhort.pl
}

Garlic (Allium sativum L.) is mainly propagated vegetatively through the cloves. This method of propagation is very conductive to the spread of virus diseases. Garlic can be infected by at least 12 viruses belonging to the four genera: Allexivirus, Carlavirus, Potyvirus and Tospovirus. Studies on the elaboration of an effective method of obtaining virus-free planting material of garlic with the use of micropropagation were conducted in the Research Institute of Horticulture. Within the conducted studies in vitro cultures of garlic were initiated, stabilized and micropropagated. The analysis aimed to determine the presence of viruses was conducted with the use of an RT-PCR technique at the initial and micropropagation stage. It was discovered that the tested plant material was infected by Onion yellow dwarf virus (OYDV), Shallot latent virus (SLV), Leek yellow stripe virus (LYSV) and Garlic common latent virus (GCLV). A research into the possibility of the elimination of above-mentioned viruses via thermo- and chemotherapy was undertaken.

For the determination of the efficiency of thermotherapy, shoots of Jarus and Ornak cultivar micropropagated on $\mathrm{B} 5$ medium containing $0.5 \mathrm{mg} / \mathrm{dm}^{3} 2 \mathrm{iP}$ and $0.1 \mathrm{mg} / \mathrm{dm}^{3} \mathrm{NAA}$ were placed in a growth chamber at a temperature of $+40^{\circ} \mathrm{C}$ with $16 / 8$ hours of light photoperiod, with a light intensity of $30 \mu \mathrm{mol} / \mathrm{m}^{2} \mathrm{~s}$ for 14 hours. The control was conducted in a phytotron under a temperature of $+20^{\circ} \mathrm{C}$. Chemotherapy was the second examined method, in which ribavirin was used for the elimination of viruses. In the conducted experiment B5 medium was applied, which in one variant was supplemented with $10 \mathrm{mg} / \mathrm{dm}^{3}$ kinetin and $0.1 \mathrm{mg} / \mathrm{dm}^{3} \mathrm{NAA}$, and in the other variant the medium was supplemented with $1 \mathrm{mg} / \mathrm{dm}^{3} \mathrm{TDZ}$ and $1 \mathrm{mg} / \mathrm{dm}^{3}$ NAA. Ribavirin in the concentration of $10 \mathrm{mg} / \mathrm{dm}^{3}$ was added to the both media. Explants placed on the above-mentioned media without the addition of ribavirin were considered as a control in the conducted experiment.

After the application of thermotherapy on the plant material of Jarus variety, 5 out of 24 explants died and in 7 of them no viruses were detected during the analysis. In Ornak cultivar, 10 out of 25 explants died and they were those which were submitted to the temperature of $+40^{\circ} \mathrm{C}$ and in 8 of them no viruses were detected during conducted experiments. In the case of chemotherapy, on B5 medium containing $10 \mathrm{mg} / \mathrm{dm}^{3}$ kinetin and $0.1 \mathrm{mg} / \mathrm{dm}^{3}$ NAA with the addition of ribavirin, 1 explant out of 5 explants of Jarus cultivar died and in 2 of them no viruses were detected. Whereas in Ornak cultivar 5 explants out of 21 plated explants died and in 2 of them no viruses were detected. After the addition of ribavirin at the same concentration to the $\mathrm{B} 5$ medium containing $1 \mathrm{mg} / \mathrm{dm}^{3} \mathrm{TDZ}$ and $1 \mathrm{mg} / \mathrm{dm}^{3} \mathrm{NAA}$, in Jarus cultivar all 15 explants survived and 4 of them were virus free. In Ornak cultivar, 5 explants out of 23 explants plated on the same medium died and in 10 of them no viruses were detected.

On the basis of the obtained results it can be concluded that both methods that we examined are characterized by similar efficiency, which is dependent on the genotype. A higher percentage of virus free explants in both methods was received in the garlic of Ornak cultivar. 


\title{
Determination of the genetic stability in long term micropropagated Fragaria $\times$ ananassa "Selva" and "Grandarosa" using AFLP markers
}

\author{
Markiewicz MoniKa, WoJTANia AgNieszKa*, IWONa SOWIK \\ Research Institute of Horticulture, Department of Applied Biology, Skierniewice, Poland \\ *agnieszka.wojtania@inhort.pl
}

In vitro propagation is one of the most important method of plant propagation, allowing for the rapid introduction of new varieties on the market. An important issue is the genetic identity of species propagated by this method, because specific conditions of in vitro cultures may generate epigenetic and genetic variation. Therefore, in plants propagated in vitro, it is necessary to control the genetic identity of plants at every stage of production.

The aim of the presented studies was an early detection of somaclonal variation in the micropropagated material of two strawberry cultivars "Selva" and "Grandarosa". They were propagated in vitro for two years on Boxus (1974) medium [2] containing benzylaminopurine (BAP), indole-3-butyric acid (IBA) and (gibberellic acid) $\mathrm{GA}_{3}$. The in vitro rooted shoots were then acclimatized to greenhouse conditions. The genetic stability was assessed using AFLP (an amplified fragment length polymorphism) molecular markers system according to Money et al. (1995 [3]) and Bachem et al. (1996 [1]). Five pairs of primers (from 15 previously tested [5]) that differentiated tested cultivars were used. The variability of shoots was evaluated in relation to reference plants (donor plants).

For all plants analyzed, AFLP primers generated a total of 236 monomorphic and 19 polymophic AFLP products. The number of bands for each primer varied between 28 (for Pst-TC/Mse-AT primer), 30 (for Pst-GA/Mse-GA primer), 76 (for Pst-CC/Mse-GG primer), 50 (for Pst-GC/Mse-GC primer) and 52 (for Pst-AG/Mse-CG primer), with an average of 47,2 bands per primer. Regarding the size of the amplified DNA fragments, it varied between $70 \mathrm{bp}$ and $800 \mathrm{bp}$. The percentage of polymorphic AFLP products in the analyzed plants in progeny lines derived from cultures that were maintained in vitro for three years, was $6.5 \%$ and $1.7 \%$ for "Grandarosa" and "Selva", respectively. In further stages of the research, we will analyze the genetic variability of strawberry during ex vitro rooting in a greenhouse and in field condition.

\section{Acknowledgements}

The study was conducted under the multiannual programme 'Actions to improve the competitiveness and innovation in the horticultural sector with regard to quality and food safety and environmental protection' of the Ministry of Agriculture and Rural Development 2015-2020.

\section{References}

[1] Bachem C., van der Hoeven R., de Brujin S. et al. (1996) The Plant J. 9(5): 745-753.

[2] Boxus P.H. (1974) Horticult. Sci. 49: 209-210.

[3] Money T., Reader S., Qu I., Dunford R., Moore G. (1995) Nucl. Acids Res. 24: 2616-2617.

[4] Murashige T., Skoog F. (1962) Physiol. Plant. 15: 473-497.

[5] Vos P. et al. (1995) Nucl. Acids Res. 23(21): 4407-4414. 


\title{
Poster no. 8
}

\section{Transformation and regeneration cucurbits plants (Cucurbitaceae)}

\author{
KaMila BŁażeJewsKa, Zbigniew TUKaJ \\ Department of Plant Physiology and Biotechnology, Faculty of Biology, University of Gdansk, Gdańsk, Poland
}

Plant production of recombinant proteins holds great potential, and may become an important delivery system, which can efficiently produce proteins in larger quantities at low production costs. Plant expression systems allow the synthesis of proteins with typical eukaryotic modifications and they are free of human pathogens and mammalian viral vectors. Moreover recombinant protein can be expressed in edible plant organs, allowing them to be administered as unprocessed or partially processed material.

The Cucurbitaceae family is formed by about 130 genera and 800 species. These important crops grow all over the world, where they are used for agronomic, healing and economic value. Furthermore cucurbits species are widely used as model plants for research, especially in studies of phloem physiology. These species are unusual in that, they possess a dual phloem transport system, that includes fascicular phloem (FP) located in vascular bundles and extrafascicular phloem (EFP) located peripheral to the vascular bundles. Biotechnology techniques have been directed to Cucurbita pepo L., C. melo L. Lagenaria siceraria (Molina) Standl and Cucumis L. because they are the economically most important crops in the world. There are few reports for the transformation and regeneration of other cucurbit especially Luffa cylindrica L. Most cucurbits seem to be relatively difficult to genetically modify. Moreover specific conditions and protocols for in vitro regeneration may be different for different species.

We describe the results of efficient transformation and plant regeneration four species of cucurbits family: Luffa cylindrica L., Cucurbita pepo L. cv Makaronowa Warszawska, Cucurbita moschata Duch., Cucurbita maxima Duch. cv Atlantic Giant using cotyledon explants excised from in vitro-grown seedlings. In our work cotyledons were cocultivated with Agrobacterium tumefaciens strain LBA4404 carrying the pRI 201-AN-GUS vector, contain $\beta$-glucuronidase (GUS) reporter gene for 4 days. We used the same medium for regeneration all cucurbit species. Fragments were placed on shoot regeneration medium containing Murashige and Skoog mineral salts and vitamins (MS), 3\% sucrose, $0.8 \%$ agar, $500 \mathrm{mg} / \mathrm{l}$ carbenicillin, $50 \mathrm{mg} / \mathrm{l} \mathrm{kanamycin} \mathrm{supplemented} \mathrm{with} \mathrm{growth} \mathrm{regulators:} \mathrm{6-benzylamino-}$ purine (BAP) and gibberellic acid (GA). Next, fragments were transferred into root induction media (RM) Murashige and Skoog mineral salts and vitamins (MS), $3 \%$ sucrose, $0.8 \%$ agar, $300 \mathrm{mg} / 1$ carbenicillin, $50 \mathrm{mg} / 1 \mathrm{kanamycin}$ supplemented with growth regulators: 6-benzylaminopurine (BAP) and indol-3-acetic acid (IAA). After adventitious root induction, plants were transferred on Murashige and Skoog mineral salts and vitamins (MS), 3\% sucrose, 0,8\% agar, $50 \mathrm{mg} / \mathrm{l} \mathrm{kanamycin} \mathrm{supplemented} \mathrm{with} \mathrm{growth} \mathrm{regulators:} \mathrm{6-benzylaminopurine} \mathrm{(BAP)} \mathrm{and} \mathrm{thidiazuron} \mathrm{(TDZ).}$

We analyzed the detection of the enzymatic activity of GUS in leaf blades and petioles collected from the regenerated plants. The analysis based on histochemical localization of $\beta$-glucuronidase, using the substrate X-Gluc, which gives a blue precipitate at the site of enzyme activity.

Our results suggest that this protocol for plant transformation and regeneration may be used at the same time for many cucurbit species. It is important for genetic engineering to identify a plant organ and protocol for rapid and effective regeneration. Use of transformed cucurbit plants will help to discover the unique system of phloem and it will also make it possible to use these plants as a molecular factory for the production of recombinant proteins. 
Poster no. 9

\title{
A complex regulatory interplay between miR156 and miR172 controls of somatic embryogenesis induction in Arabidopsis
}

\author{
Katarzyna NowaK *, AnNa WóJcik, Maegorzata Danuta GaJ \\ Department of Genetics, Faculty of Biology and Environment Protection, University of Silesia, Katowice, Poland \\ *katarzyna.nowak@us.edu.pl
}

The embryogenic transition of somatic cells in plants requires extensive reprogramming of the cell transcriptome. Congruently, the extensive modulation of genes with a regulatory function including the genes encoding the transcription factors and miRNAs were indicated to the control induction of a somatic embryogenesis in Arabidopsis. Among SE-modulated miRNAs, miR156 and miRNA172 were reported. The level of these miRNAs modulate the embryogenic potential of the explants. Thus, the involvement of miR156 and miRNA172 in the regulation of the SE in Arabidopsis was assumed.

miR156 and miR172 are regulatory related and during the in vivo development they control the vegetative to reproductive phase transition through targeting the genes, respectively $S P L S$ and $A P 2 / A P 2$-like (TOE1, 2, 3, SMZ, $S N Z$ ). To reveal the genes targeted by miR156 and miR172 in SE of Arabidopsis we analyzed the expression of the candidate $T F S$ in embryogenic cultures of the MIM lines with the abolished function of the studied miRNA. The elevated expression of SPL2, 3, 9, 10,11 and 13 in the embryogenic culture of the MIM156 line implied that miR156 might control these TFS during SE. Two of them, SPL9 and SPL10, were reported to control MIRNA172 genes in vivo. Hence, we analyzed the expression level of MIR172 genes in the SE-induced MIM156 explants. The increased level of primiR172A, B, Din the MIM156 culture suggested that miR156-SPL9/10 module might positively control expression of MIR172 genes in SE. Moreover we found that the increased transcription of the MIR172 genes was associated with the decreased level of the mature miR172 product in the MIM156 culture. This apparent inconsistency confirmed the posttranscriptional processes to be involved in the regulation of miRNA during SE. The expression profiling of the candidate miR172 targets in the embryogenic culture of the MIM172 line indicated an accumulation of $T O E 1$ and $A P 2$ transcripts and that result suggested a regulatory relation between miR172 and TOE1/AP2 during SE.

The regulatory interplay between miR156, miR172 and a known SE regulator - AGL15 were reported in plant development, thus we analysed the relationship between miR156, miR172 and AGL15 during SE induction. The results showed an accumulation of the $A G L 15$ transcript in MIM172 and $A P 2$-overexpression line cultures, thus suggesting that miR172 may regulate the expression of $A G L 15$ via targeting $A P 2$ during SE in Arabidopsis.

In addition, we analysed the expression level of mature miR156 and MIRNA156 genes in the SE-induced explants of the $A G L 15$ overexpression line. The analysis showed that the overexpression of $A G L 15$ resulted in an elevated level of the primary transcripts of MIRNA156 genes and the reduced accumulation of the mature miR156. Hence, the complex posttranscriptional regulation of pri-miRNA might account for the observed inconsistency. Relevant to this assumption, $A G L 15$ appears to impact the genes involved in miRNA biogenesis as we observed the inhibition of HEN1, SERRATE and DCL1 in AGL15 overexpression line. However, this hypothesis needs further experimental verification.

In conclusion, the study infers a complex regulatory interplay between the miR156-SPL9/10 and miR172AP2/AGL15 modules during SE. The SE-involved regulatory circuit involves a feedback regulation of miR156 by AGL15, a target of miR172, and further experiments are required to verify the proposed interactions.

This work was supported by a research grant for Young Scientist funded by the University of Silesia in Katowice. 


\title{
The influence of catecholamines on the expression levels of selected genes of the phenylpropanoid pathway in potato (Solanum tuberosum)
}

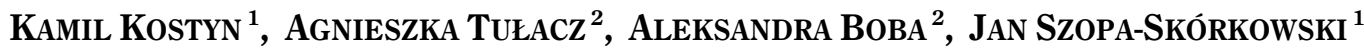 \\ ${ }^{1}$ University of Life Sciences in Wrocław, Wrocław, Poland \\ ${ }^{2}$ University of Wrocław, Wrocław, Poland \\ *gusia5614@wp.pl; aleksandra.boba@uwr.edu.pl; kamil.kostyn@upwr.edu.pl; szopa@ibmb.uni.wroc.pl
}

Catecholamines are biogenic aromatic monoamines widespread in animals, where they are neuromodulators and stress hormones. These compounds have also been identified in plants, though their function in these organisms was not fully recognized. It is known that after binding with other phenolics they can reinforce the cell wall either by constituting a physical barrier for pathogens or increasing their antioxidative potential. Catecholamines are strong antioxidants and they can neutralize free radicals (including those resulting from pathogen infection). In plants, catecholamines are also precursors for the biosynthesis of alkaloids (e.g. morphine, mescaline) or pigments (betalains, melanins). However, there are data indicating that besides the structural function, catecholamines may play a more complicated role of regulatory molecules in plants. In animals, one of the most identified hormonal functions of catecholamines is their influence on sugar metabolism (glycogen breakdown). In plants, starch fulfills an analogous function to glycogen - which is the storage of sugar and an energy reservoir. Studies on catecholamines in potato suggest that in plants catecholamines show some resemblance in functions - they influence the starch breakdown and lead to increased contents of soluble sugars. In other studies (i.a. in tobacco) catecholamine were found to exercise influence on plant growth, probably resulting from their interaction with phytohormones. However, so far there have been no data that would confirm the regulatory activity of catecholamines in plants. Because of the fact that catecholamines in animals are strictly connected with their response to stress, we wanted to analyze if such activity of this group of compounds was present in plants. It is known that under various stress conditions secondary metabolites are produced and phenolic derivatives are among them. Among different functions, they possess a high antioxidant capacity to prevent the effect of a stressor. Although the very catecholamines were shown to act as structural molecules and are incorporated into the cell wall structure in the form of amides with phenolic acids in plants' response to stress conditions, there may also be a regulatory function of these compounds that governs the activity of a whole ensemble of genes related to the biosynthesis/metabolism of phenolics. Therefore, in our experiment four-week-old potato plants in in vitro culture were submitted to catecholamine treatments and the tissue samples were collected at several timepoints after the treatments. Subsequently, an analysis of the transcript level of selected genes of the phenylpropanoid pathway was performed. At the same time, we measured the corresponding metabolite levels and the data were compared. Activation of the analyzed genes accompanied by changes in the metabolite contents were observed. Potato is an important crop plant and a better insight into the mechanisms of the regulation of plant physiological processes may result in new projects like the generation of more resistant plants that are especially important for application in food industry. 
Poster no. 11

\title{
Optimization of carrot protoplasts fusion parameters using fluorescent proteins
}

\author{
Tomasz Oleszkiewicz * Julianna Siwek, Miron Gieniec, Magdalena KlimeK-Chodacka, \\ Katarzyna MaćKowska, Ewa Grzebelus, RafaŁ BarańSKI
}

Institute of Plant Biology and Biotechnology, Faculty of Biotechnology and Horticulture

University of Agriculture in Krakow, Krakow, Poland

*t.oleszkiewicz@urk.edu.pl

Somatic hybrids can be obtained by using various methods, including protoplast electrofusion. The choice of the optimal electrofusion conditions is essential for achieving an efficient fusion rate allowing a successful hybrid cell development. Usually, protoplasts derived from hybrid parental components are indistinguishable. Thus optimization of the electrofusion process is based on scoring the hybrid rate that requires a prolonged cell culture and many repetitions of the experiments with modified parameters of electrofusion. A simplified optimization can be done when protoplasts of both parental components are visually distinguishable, which makes fast assessment of the fusion rate possible immediately after electrofusion without any need for a further cell culture. Cell markers for this purpose must be easily identifiable, have no adverse effect on the cell development, and their identification in a hybrid cell must be feasible.

The aim of this study was to develop a system for monitoring protoplast electrofusion that would facilitate to optimize the fusion parameters. For this purpose we transformed the callus derived from two cultivars, "Koral" and "Amsterdamska", and a doubled haploid DH1 line and created a set of transgenic lines with cells expressing different fluorescent proteins. Four vectors used for transformation contained genes coding for different fluorescent proteins, i.e., CFP, GFP, YFP, and mCherry, with localization signals to mitochondria. After Agrobacterium tumefaciens-mediated transformation only callus lines that showed intense and homogeneous fluorescence were selected. Protoplast were isolated from a well growing callus tissue using the enzymatic cell wall digestion. Up to $96 \%$ of isolated protoplasts exhibited fluorescence when excited using a fluorescence microscope. In preliminary experiments we screened various direct current voltages $(2-3.5 \mathrm{kV})$ and impulse times $(40-100 \mu \mathrm{s})$ applied to protoplasts isolated from one line to determine a range of the least damaging conditions but ensuring cell fusion. Then the selected sets of parameters were applied to mixtures of protoplasts derived from two components possessing proteins emitting fluorescence of different wavelengths. The protoplast fluorescence of each component was recorded separately after using dedicated filter sets. Hybrids were identified when the cell exhibited fluorescence in both wavelength characteristics for fusion components. During the electrofusion most cells were damaged and only some remained intact and could fuse. The percentage of identified hybrids was up to $18 \%$ but it was generally lower, around a few per cent points, and it depended on which pair of cell lines was used.

Based on the hybrid frequency we selected $50 \mathrm{~V} / 60 \mu$ s and $60 \mathrm{~V} / 50 \mu$ s conditions as best parameters of a direct current impulse for successful electrofusion. Our results show that the use of cell lines expressing fluorescent proteins provides a convenient material for monitoring and optimizing the process of protoplasts fusion. It is worth emphasising that the use of fluorescent proteins as markers allowed non-destructive observations of cells during the fusion in real time.

\section{Acknowledgements}

The research was financed from the funds for basic research on crop improvement granted by the Polish Ministry of Agriculture and Rural Development for the years 2016-2020. 


\title{
Poster no. 12
}

\section{Possibility of Lupinus angustifolius L. ovule development in in vitro cultures with applying wide crossing}

\author{
Renata GaleK ${ }^{1}$, Ewa Sawicka-Sienkiewicz ${ }^{1 *}$, AnNa Maciejewska-Hoza ${ }^{1}$, \\ Bartosz KoZAK $^{1}$, AdEla AdAMUS ${ }^{2}$, AgNiESZKa KIElKowsKa ${ }^{2}$ \\ ${ }^{1}$ Department of Genetics, Plant Breeding and Seed Production, \\ Wrocław University of Environmental and Life Sciences, Wrocław, Poland \\ ${ }^{2}$ Unit of Genetics, Plant Breeding and Seed Science, University of Agriculture in Krakow, Kraków, Poland \\ *ewa.sawicka-sienkiewicz@upwr.edu.pl; a.adamus@ogr.ur.krakow.pl
}

Attempts to obtain haploids in Fabaceae against the background of the achievements of other species are still at the stage of preliminary research. More than several hundred species belonged to this family, but only in 16 species were regenerated single haploid plants by androgenesis using anther cultures or isolated microspores. The work carried out so far has been focused on increasing the efficiency of the haploidization process. The aim of the study was to stimulate the development of the potential structures of an embryo sac of narrow-leafed lupin ( $2 \mathrm{n}=40$ chromosomes) after previous pollination by using pollen grains from of another species - Lupinis mutabilis ( $2 \mathrm{n}=48$ chromosomes). Four genotypes of narrow-leafed lupin were the objects of experiment: "Graf", "Emir", "Karo", LAE-1. In the first stage, the behavior of the L. mutabilis pollen on the stigma was checked in in vivo condition (at the presence of pollen grains on stigma, germination of pollen grains on stigma and growing in the style, the presence of a pollen tube in ovule -48 and 96 hours after pollination). The pistils have been fixed in Carnoy solution and analyzed using a fluorescence microscope after staining with aniline blue. At the same time anthers were removed from the mother plants before flowering and then two days later after this treatment fresh pollen from Andean lupine was applied. Spraying with the $2.4-\mathrm{D}$ solution $\left(100 \mathrm{mg} / \mathrm{dm}^{3}\right)$ was carried out during three consecutive days. The ovules were isolated after 7-10 days after pollination, and then were disinfected and placed in in vitro culture. As a reference point, isolated ovules from four genotypes of narrow-leafed lupin were obtained from pistils which were not pollinated of $L$. mutabilis pollen. Ovule cultures were put on the same media. The MS medium with macro and micronutrient modifications was applied after its supplementation with plant growth regulators $\left(\mathrm{mg} / \mathrm{dm}^{3}\right): 2.02 .4-\mathrm{D}, 0.5 \mathrm{kin}$ and $120 \mathrm{~g}$ of sucrose. Four days after isolation, the ovules were transferred to MS medium, but with the addition of $0.5 \mathrm{BAP}$ and $0.1 \mathrm{NAA}\left(\mathrm{mg} / \mathrm{dm}^{3}\right)$, because they began to turn brown. Within two weeks, the regeneration of the ovules was noted. The development of callus tissue with granular structure has been observed in the culture of ovules. More spherical structures began to form on parts of explants. Most changes were founded in "Emir" (74\% of explants with changes), followed by "Karo", LAE-1 (15-45\%) and "Graf" (5\%). Regeneration of the granular callus tissue was also observed in isolated ovules which were not treated with $L$. mutabilis pollen. Only 5-10\% of explants "Emir" and "Karo", respectively, showed the regeneration ability. The obtained results concerning the behavior of $L$. mutabilis pollen on the stigma showed that it was still present on the stigma after $48 \mathrm{~h}$ and $96 \mathrm{~h}$ from pollination, and, additionally, a few pollen tubes were observed in the style and reached the ovules. In the future, after obtaining regenerated plants and applying appropriate diagnostic techniques, it will be possible to explain which kind of mechanisms plays the main role in the regeneration of ovules in in in vitro cultures and whether they could be effective in obtaining potential haploids plants or $\mathrm{DH}$ lines in narrow-leafed lupin. 
Poster no. 13

\title{
Assessment of metal nanoparticles-induced morphological and physiological changes in in vitro cultures of rapeseed (Brassica napus L.)
}

\author{
Magdalena Tomaszewska-Sowa ${ }^{1}$, AnetTa Siwik-ZIOMEK ${ }^{2}$, AnNa Figas ${ }^{1}$, Karol Bocian ${ }^{1}$ \\ ${ }^{1}$ Depertment of Agricultural Biotechnology, University of Science and Technology in Bydgoszcz, Poland \\ ${ }^{2}$ Department of Biogeochemistry and Soil Science University of Science and Technology in Bydgoszcz, Poland
}

Due to their specific structure nanoparticles such as: nanosilver, nanocopper and nanogold can be characterized by a whole array of interesting features. They manifest very strong antibacterial and antifungal properties. The impact of metal nanoparticles on the environment is not clear. There is no information on the interaction of nanoparticles (NPs) with plants. To increase our understanding of the mechanisms involved in plant responses to metal nanoparticles we determined the morphological and physiological changes induced in rape plants (Brassica napus L.). Sterile rape seeds were inoculated onto the MS medium (sterilized and unsterilized) containing metal nanoparticles (silver, copper and gold) in $20 \mathrm{mg} / \mathrm{kg}$ concentration. Significant differences in B. napus seedlings growth were reported in each case when metal nanoparticles were used and in the case when the medium was differentiated for the autoclave sterilization process. The analysis of the numerical results allowed to conclude that the high temperature and pressure that occur during the process of media sterilization affects the reduction of the biological activity of nanoparticles. The effect of nanoparticles on the germination processes depended on the type of metal. Silver and gold nanoparticles did not inhibit the germination process. Nanoparticles in low concentration did not affect the roots length. However, there was an increase in the length of the root in the presence of nanosilver on an unsterilized medium. The seedlings grown on a medium supplemented with nanoparticles were significantly shorter in comparison to the controls. Nanosilver without autoclave sterilization reduced the height of plants to 5.87 . In the present investigation, we also studied influence nanoparticles on different physiological parameters such as total chlorophyll and carotenoid content and the activity of antioxidant enzyme- catalase. The total chlorophyll and carotenoid content decreased after exposure to metal nanoparticles. This effect was particularly noticeable after the application of nanogold. In the presence of nanogold the chlorophyll content decreased by 33\%. The lowest decrease in chlorophyll concentration, about $17 \%$, was observed when nanocopper was used. A similar effect of a reducing concentration under the influence of nanogold was obtained for carotenoids. The amount of carotenoids after using this factor decreased by $28 \%$. Nanoparticles of gold also increased the activity of catalase when compared with the controls. The oxidative stress in nanoparticle-treated plants was reflected by an increase of catalase activities in the seedlings of rapeseed plants. Thus, it may be stated that plants protect the cellular and sub-cellular systems against the cytotoxic effects of active oxygen radicals with antioxidative enzymes (catalase). 


\title{
Poster no. 14
}

\section{The effect of the medium composition and the embryo age on the development of hybrid embryos of Solanum lycopersicum L. $\times$ S. sisymbriifolium Lam. cultured in vitro}

\author{
Eukasz PiosiK *, Maria Ruta-Piosik, Maciej ZenkTeler, Elżbieta ZenkTeler \\ Department of General Botany, Faculty of Biology, Adam Mickiewicz University in Poznań, Poland \\ *1.piosik@amu.edu.pl
}

Solanum lycopersicum, a widely cultivated and popular vegetable crop is affected by diseases caused by numerous factors. To improve the resistance of plants to several pathogens, the breeders try to transfer the resistant genes that are present in the wild relatives. S. sisymbriifolium, also known as a sticky nightshade, is recognized as a species resistant to various pathogens i.e. nematodes or bacteria which are known to cause diseases of the Solanaceae vegetable crops. Several authors have reported that the resistance against certain pathogens was successfully transferred from $S$. sisymbriifolium to e.g. S. melongena hybrids. Such resistance would be very desirable for the cultivated tomatoes, so we decided to cross $S$. lycopersicum with $S$. sisymbriifolium in order to obtain hybrid regenerants. What is more, hybrids of $S$. lycopersicum $\times S$. sisymbriifolium have never been described.

Male sterile $S$. lycopersicum plants and fertile $S$. sisymbriifolium MM-668 ones (sourced from INRA UR1052) were delivered by Rijk Zwaan Company and used as the components of crossing. As a result of the interspecific cross, few-celled and globular embryos were produced. However, due to the developmental disturbances, the growth of embryos was inhibited in vivo at the globular stage. The embryos were often abnormally, highly oversized and the endosperm was insufficient. To assure further development of the hybrid embryos, we used the embryo-rescue method. For the culture purposes we gathered the fruits produced 14-35 days after pollination. The enlarged ovules were aseptically dissected from the fruits after removing the jelly-like coating. The globular embryos isolated from the ovules were transferred on the solid, modified MS media supplemented with plant growth regulators and additives. In the first weeks of the investigations, the embryos were nursed in vitro on the tomato endosperm isolated around 15 days after selfing. Because both embryos and endosperm started degenerating during the first days of culturing, we decided to put the globular embryos directly on the media. The embryos were cultured in Petri dishes which were kept in a climate chamber.

The results obtained from the culture depended mainly on the media composition and the age of the cultured embryos. Over $17 \%$ of embryos (mainly those isolated 20-22 DAP) produced morphogenic and green callus, mainly on the media with the addition of zeatin and 2,4-D and at various sucrose concentrations (from 3-9\%). Such calluses proliferated intensively and hybrid plants were regenerated from some of them. Only four cultured embryos (isolated around $25 \mathrm{DAP}$ ) grew directly into the plantlets, however they died during the acclimatization process. Over three quarters of the cultured embryos (mainly younger than $20 \mathrm{DAP}$ and older than $25 \mathrm{DAP}$ ) never developed or only highly oversized (even 3-4 times) on the applied media, which indicates the presence of strong postzygotic barriers. More details concerning the results of the in vitro cultures of the hybrid embryos obtained will be presented on the poster. 
Poster no. 15

\title{
The in vitro culture of white lupin embryos for the shortening of the generation cycles in the production of homozygous lines by the single seed descent technique
}

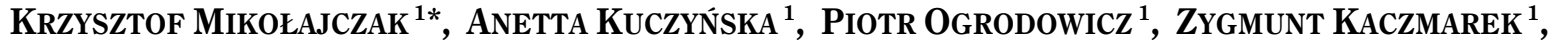 \\ HANNA ĆWIEK-KUPCZYŃSKa ${ }^{1}$, MARIa SURMa ${ }^{1}$, TAdEUSZ AdAMSKI ${ }^{1}$, WOJCIECH ŚWIĘCICKI ${ }^{1}$, PaWEe BarzYK ${ }^{2}$, \\ RENATA TRZECIAK ${ }^{1}$, Michą KeMPA ${ }^{1}$ \\ ${ }^{1}$ Institute of Plants Genetics Polish Academy of Sciences, Poznań, Poland \\ ${ }^{2}$ Poznań Plant Breeders Ltd., Tulce, Poland \\ *kmik@igr.poznan.pl; pawel.barzyk@phr.pl
}

Lupins are important agricultural crops in Central and Northern Europe because their seeds are a great source of fat (10-15\%), protein (30-40\%) and carbohydrates (12-16\%). White lupin (Lupinus albus L.) is basically a selfpollinating species. For the purpose of breeding, new cultivar homozygous lines should be developed. The production of such lines in a temperate climate is a multi-year process. To accelerate this process, the single seed descent (SSD) technique combined with an in vitro culture of embryos is proposed.

The aim of the study was to establish the optimal in vitro culture conditions for embryos dissected from immature seeds, which is the first step in the project aimed at shortening the generation cycles in the development of homozygous lines. In the first experiment, two varieties, Boros (a self-completing type of growth) and Butan (a normal type of growth), and four temperature regimes: 1) $8^{\circ} \mathrm{C}$; 2) the first week $16^{\circ} \mathrm{C}$, then $8^{\circ} \mathrm{C}$; 3) the first week $20^{\circ} \mathrm{C}$, then $20 / 18^{\circ} \mathrm{C}$; 4$) 16^{\circ} \mathrm{C}$ during whole culture period, were applied. In each temperature treatment, the first week of the culture was in the dark, and in the next weeks a photoperiod of $8 / 16 \mathrm{~h}$ (day/night) was maintained. Embryo development was evaluated by the observation of shot and root length after 7, 14 and 21 days of culture. Plants of ca. $5 \mathrm{~cm}$ in height with well-developed roots (after about 30 days of culture) were transferred to pots. Then, plant survival ex vitro was observed on the $30^{\text {th }}$ day after planting.

The data for shoot and root length were statistically processed using the analysis of variance (ANOVA), in which genotypes, temperature treatments and culture duration (term of measurement), as well as corresponding interactions were taken into account as the sources of variation. The results of the experiment showed that at lower temperatures the plants developed slowly, but their survival ex vitro was higher (about 60\%) than of those developed at higher temperatures (survival about 28\%). The results also indicated significant differences in the rate of embryo development between varieties of normal and self-completing type of growth. Therefore, an additional experiment covering 10 varieties, eight self-completing (breeding lines 95181, 95180, 95442, 95179, S 95144, 95177, 95673 and cv. Boros) and two of normal growth (N-BAC and cv. Butan), was performed. The results of this experiment confirmed the very slow development of embryos of self-completing plants. The explanation of the causes of this phenomenon requires further research. These studies are a valuable source of information and will be continued as a part of the project, task 2.3: The application of biotechnological methods to increase and accelerate biological progress in leguminous plant breeding.

\section{Acknowledgements}

The studies were supported by the Ministry of Agriculture and Rural Development, the National Multi-Year Project (2016-2020), Resolution No. 222/2015, 15 December 2015. Task 2.3. Application of biotechnological methods to increase and accelerate biological progress in leguminous plant breeding. 


\title{
Poster no. 16
}

\section{Selection of the fluorescent labels useful for monitoring of protoplast fusion}

\author{
Katarzyna Mackowska, Edyta Florek, Ewa Grzebelus * \\ Institute of Plant Biology and Biotechnology, University of Agriculture in Krakow, Kraków, Poland \\ * ewa.grzebelus@urk.edu.pl
}

The somatic hybridization technique allows to combine the nuclear and cytoplasmic genomes and thus receive inter- and intraspecific hybrids. Although, protoplasts fusion is potentially useful tool, one of the major problems is lack of effective markers for somatic hybrids identification and selection. The selection system may base on specific morphological and physiological properties of the cells. However, the use of these markers is not always applicable due to demand of unique cell lines often difficult to obtain. One of the efficient methods for overcoming these limitations is fluorescent labeling. The applicable fluorescent dyes should be non-toxic for the cells, easy detectable and present in high fluorescent intensity. Additionally, the persistence of dyes in the cells and lack of diffusion from one protoplast to another during electrofusion are important selection criteria. In this study three fluorescent dyes i.e. fluorescein diacetace (FDA, yellow-green fluorescence), rhodamine B isothiocyanate (TRITC, red fluorescence) and scopoletin (blue fluorescence) have been used as potential florescent labels for monitoring of protoplast fusion.

The objectives of the studies were as follow: 1) evaluation of fluorescent dyes stability in the protoplast cells before and after electric field treatment; 2) investigation harmful effect of fluorescent dyes on protoplasts regeneration; 3) selection of a combination of two label allowing to effective detection of heterofusion products.

Leaf-derived protoplasts of $D$. carota subsp. sativus were stained with scopoletin $(100 \mu \mathrm{g} / \mathrm{ml})$ during overnight enzymatic digestion of the donor tissue while with FDA $(75 \mu \mathrm{g} / \mathrm{ml})$ and TRITC $(2.5 \mu \mathrm{g} / \mathrm{ml})$ just after protoplast purification. The stained protoplasts were washed in $0.4 \mathrm{M}$ mannitol and incubated on ice or at room temperature and then the stability and fluorescent intensity of dyes were evaluated under inverted fluorescent microscopy. After incubation on ice the effect of electric field treatment on diffusion of dyes was evaluated by application use three types of direct current impulses ( $60 \mu$ s and $50 \mu$ s with voltage $2.5 \mathrm{kV} / \mathrm{cm}, 50 \mu \mathrm{s}$ with voltage $3.0 \mathrm{kV} / \mathrm{cm}$ ). Additionally, differently stained protoplasts were mixed in each combination and then the quality of differential staining was assessed. Subsequently protoplasts were cultured according to Grzebelus et al. (2012) [1] and after 2 months the level of protoplast regeneration was evaluated.

The differences in fluorescence intensity for individual dyes were observed and for some of the cells stained with the scopoletin weak fluorescence were noted. The highest level of label diffusion from protoplasts was observed for scopoletin, what was manifested by decreasing of fluorescence intensity and increasing of background. Additionally, incubation of the protoplasts on ice successfully reduced dyes diffusion from the cells and in the consequence the background. Diffusion of the labels observed after electric field treatment was strongly dependent on pulse voltage and duration. Analysis of regeneration capacity showed that the used labels were not toxic for the protoplasts and allow for their growth and development. Based on the above analysis FDA and TRITC were selected as the most suitable dye set for differential staining of protoplasts in early selection of heterofusion products.

\section{Acknowledgements}

The research was financed from funds for basic research on crop improvement granted by the Polish Ministry of Agriculture and Rural Development in the years 2016-2020.

[1] Grzebelus E., Szklarczyk M., Baranski R. (2012) PCTOC 109: 101-109. 


\title{
The influence of DNA hypomethylation on microspore embryogenesis effectiveness in winter triticale $(\times$ Triticosecale Wittm.)
}

\author{
Monika Krzewska *, Anna Nowicka, Ewa Dubas, PrzemysŁaw Kopeć, \\ Katarzyna JUZoń, KaMil ZiEliński, Sabina Malaga, Iwona ŻUR
}

The Franciszek Górski Institute of Plant Physiology, Polish Academy of Sciences, Kraków, Poland

Microspore embryogenesis (ME) is an example of plant cell totipotency, where male gametophytic cells (microspores) switch their developmental pathway towards haploid embryo formation. This remarkable process is considered one of the fastest and simplest routes to obtain haploid and doubled haploid plants (DHs), highly appreciated in breeding molecular studies and genetic engineering. However, high ME effectiveness is achieved only under certain circumstances as a consequence of a specific stress treatment and it is controlled by an interaction of genetic and physiological factors. The application of $\mathrm{ME}$ has considerable limitations because the mechanisms of microspore reprogramming and further development and differentiation of produced embryo-like structures are not yet completely elucidated.

Methylation is a post-replication modification of DNA, which regulates gene expression during the development and differentiation of cells. In plants, DNA methylation plays an important role in different physiological processes such as acclimation, embryonic development, senescence and flowering. High level of DNA methylation (hipermethylation) of some chromatin fragments causes partial or complete gene silencing. Reduction of DNA methylation (hypomethylation) through the use of demethylating agents causes changes in chromatin conformation and increases the accessibility of the genome to the transcription machinery. The effect of 5-azacytidine (AzaC), a DNA methylation inhibitor, on ME effectiveness was investigated in anther and isolated microspore (MKS) cultures of winter triticale ( $\times$ Triticosecale Wittm.). Two DH lines of triticale, signi cantly different with respect to embryogenic potential, were used as the object of the study.

Four days of $10 \mu \mathrm{M} \mathrm{AzaC}$ tillers treatment significantly increased ME induction effectiveness in the highly responsive DH line (DH28). As a result, the average number of ELS was 4- and 2-fold higher in MKS and anther cultures, respectively, in comparison to the controls. The $\mathrm{AzaC}$ treatment also slightly increased the final regeneration ability in anther cultures (from 5 to 6 green plants per 100 anthers) and caused an almost 4 -fold increase in the number of green plants regenerated in MKS cultures.

However, in the low responsive $\mathrm{DH}$ line (DH19), the AzaC treatment had a negative effect on ME induction. Although the yield of isolated microspores and their viability was higher after the AzaC treatment than in control cultures, it decreased the average number of ELS produced in anther cultures from 2.7 to 0.7 and completely inhibited ELS formation in MKS cultures.

The presented results provide a new insight into the role of epigenetic modifications in a microspore embryogenesis and suggest a potential usage of DNA methylation inhibitors to improve the efficiency of this process, thus widening its application in biotechnology and breeding programs.

The research was performed in the frame of projects NN310452638 and 2015/18/M/NZ3/00348 financed by the National Science Centre and supported by institutional funding of the Institute of Plant Physiology Polish Academy of Sciences, project no. T1Zb2/2016. 


\title{
Poster no. 18
}

\section{Photomorphogenesis of androgenic cultures of winter wheat and winter barley using Intelligent Lighting Systems for the Plant Production Industry (ISOR)}

\author{
MAGDALENa TACIAK ${ }^{1 *}$, Michat ROKICKI ${ }^{1}$, TOMASZ SZYMAŃSKI ${ }^{1}$, \\ MARIA ZdZIECHOWSKA-DUDEK ${ }^{1}$, KAMIL CISZAK $^{2}$, STANISEAW KARPIŃSKI ${ }^{2}$ \\ ${ }^{1}$ Poznan Plant Breeding Sp. z o.o.,Wiatrowo Plant Breeding Branch, Wagrowiec, Poland \\ ${ }^{2}$ Department of Plant Genetics, Breeding and Biotechnology, Faculty of Horticulture, \\ Biotechnology and Landscape Architecture, Warsaw University of Life Sciences - SGGW, Warszawa, Poland \\ *magdalena.taciak@phr.pl
}

The aim of the study was to increase the efficiency of the derived plant material in in vitro conditions by choosing appropriate lighting conditions. Two genotypes of winter wheat and winter barley, previously characterized as susceptible and resistant to the induction of developmental processes in in vitro cultures, were selected for the experiment purpose. Seeds were germinated in the greenhouse and seedlings were then vernalized for 8 weeks and then grown in a greenhouse chamber for $4-5$ weeks. Androgenic cultures of winter wheat and winter barley were carried out in accordance with the methodology used in the in vitro laboratory of Poznan Plant Breeding Sp. z o.o. Spikes with microspores in the late uninucleate stage were collected and cold pre-treated $\left(4^{\circ} \mathrm{C}\right)$ to stimulate androgenetic development. Anthers were isolated aseptically and transferred onto Petri dishes containing $\mathrm{C} 17$ induction medium (Wang and Chen, 1983) for wheat and FHG induction medium (Hunter, 1988) for barley. In all experiments, cultures were grown in the dark at $26^{\circ} \mathrm{C}$. Androgenic structures (calli and embryos) of approximately $1 \mathrm{~mm}$ in diameter were successively transferred onto the regeneration medium. The experiment was performed at a temperature of $22^{\circ} \mathrm{C}$, at $16 \mathrm{~h}$ photoperiod using standard lighting and using the intelligent ISOR lighting system imitating natural changes in light intensity with a suitable photon beam: white $50 \mu \mathrm{E}$ with a wavelength of $440-700 \mathrm{~nm}$, blue $150 \mu \mathrm{E}$ with a wavelength of $405-455 \mathrm{~nm}$, red $150 \mu \mathrm{E}$ at $620-660 \mathrm{~nm}$ and infrared $2 \mu \mathrm{E}$ at $730 \mathrm{~nm}$. In experiments with photomorphogenesis of haploid winter wheat plants, 12000 anthers were placed on the induction medium. The analysis of the results obtained showed a significant difference in the number of regenerants of winter wheat obtained, depending on the type of lamps used. In the case of ISOR lamps, regeneration of 28 plants was obtained, whereas in the case of the control, it regenerated 16 plants. In the experiment on photomorphogenesis of winter barley plants, a total of 12600 anthers were isolated in the induction media. 40 regenerated plants were obtained after using ISOR light and 28 green plants under standard lighting.

The results presented above refer to the experiment carried out as part of research task $3 / 2017$. This work was supported by the Applied Research Programme of the National Centre for Research and Development - PBS/A9/37/2015. 
Poster no. 19

\title{
Endogenous phytohormones related to the efficiency of oat (Avena sativa L.) doubled haploid production
}

\author{
Kinga DziUrka *, Michą DziUrka, Marzena Warchol, Ilona CzyczyŁo-Mysza, \\ IZabela MarcińsKa, ANGElika Noga, Kamila KaPloniaK, EdYTa SKRZYPeK \\ The Franciszek Górski Institute of Plant Physiology, Polish Academy of Sciences, Kraków, Poland \\ *k.dziurka@ifr-pan.edu.pl
}

Oat (Avena sativa L.) is an economically important crop species. Double haploids are frequently used in modern plant breeding in order to shorten the process of creating new cultivars. The conversion of haploid embryos into plants is the stage that limits the efficiency of oat doubled haploid production by wide crossing with maize. The aim of this study was to examine the association of endogenous phytohormones with the germination capacity of oat haploid embryos.

The experiment was performed on oat F1 progeny derived from Strzelce Plant Breeding Ltd. Panicles at the right stage of maturity were emasculated, then pollinated with maize and treated with a 2,4-dichlorophenoxyacetic acid $(2,4-D)$ solution. Three weeks after pollination, enlarged ovaries were collected, surface sterilized and haploid embryos were isolated and placed on 190-2 medium [3] with $0.5 \mathrm{mg} / \mathrm{dm}^{3}$ kinetin, $0.5 \mathrm{mg} / \mathrm{dm}^{3}$ 1-naphthaleneacetic acid (NAA), 9\% maltose, $0.6 \%$ agar and $\mathrm{pH} 6.0$. The ovaries in which haploid embryos were developed (OE) and those in which embryos did not develop (OWE) were collected and analyzed for hormonal composition by the HPLCMS/MS method. Haploid embryos were cultured in a $16 \mathrm{~h}$ photoperiod, at light intensity of $130 \mu \mathrm{mol}$ (photons) $\mathrm{m}^{2} / \mathrm{s}$ and at $20 / 17^{\circ} \mathrm{C}$ (day/night).

Of the 8 oat (F1) genotypes, 5794 florets were emasculated. Three hundred forty six haploid embryos were obtained. The germination rate of haploid embryos varied between genotypes from $0.43 \%$ (STH 4.4729) to 5.67\% (STH 4.4690). There were differences between the hormonal composition of $\mathrm{OE}$ and OWE. A significantly higher indole-3-acetic acid, trans-zeatin and kinetin contents were noted in $\mathrm{OE}$ (403.9, 47.0 and $45.2 \mathrm{pmol} / \mathrm{g} \mathrm{DW}$, respectively) in comparison to OWE $(245.9,29.4$ and $5.5 \mathrm{pmol} / \mathrm{g} \mathrm{DW}$, respectively). On the other hand, 4-Cl-IAA was detected in the OWE (182.8 pmol/g DW) while in OE it was not present. There was no relationship between the auxin content in oat ovaries and the frequency of haploid embryos and their germination ability. In contrast, the presence of cytokinins: trans-zeatin and kinetin in the ovaries was strongly negatively correlated with the examined characteristics. However, the presence of cytokinin in the medium did not disturb the conversion of oat haploid embryos into plant [2]. The positive correlation between the $\mathrm{GA}_{1}$ level in oat ovaries and the frequency of haploid embryos and haploid embryos germinating was observed.

The obtained data suggest that the hormonal composition of ovaries influenced the development of the haploid embryos.

\section{Acknowledgments}

The research was partly funded by the National Centre for Research and Development, PBS3/B8/17/2015

\section{References}

[1] Murashige T., Skoog F. (1962) Physiol. Plant. 15: 473-497.

[2] Noga A., Skrzypek E., Warchoł M. et al. (2016) In Vitro Cell. Develop. Biol. Plant 52: 590-597.

[3] Wang X.Z., Hu H. (1984) Plant Sci. Lett. 36: 237-239. 


\title{
Poster no. 20
}

\section{In vitro and in vivo induction of chromosome doubling of Brassica napus L. haploids}

\author{
LaUREnCJa SzaŁa, Katarzyna SoSNOWSKa \\ Plant Breeding and Acclimatization Institute - National Research Institute, Poznań Division, Poland \\ *1szala@nico.ihar.poznan.pl
}

The development of doubled haploid (DH) plants trough a culture in vitro e.g. a microspore culture is an important and efficient method for the production of fully homozygous lines in a short time. This method has been found wide application in a genetic study and breeding programmes. The haploid technology for Brassica napus is probably the most highly developed system among flowering plant species. Since the derived-microspore plants originate from gametes, they have usually a single set of chromosomes and in the vast majority they are haploids. In the case of oilseed rape (Brassica napus L.) spontaneous chromosome doubling frequency is low and ranges from $10 \%$ to $40 \%$ depending on the genotype. This number of diploids is unsatisfactory. Chromosome doubling of microspore-derived plants is an important factor in the practical application of androgenesis in vitro in an isolated microspore culture because breeding programs require a large number of genetically stable, fertile homozygous doubled haploid plants. Therefore, the use of effective techniques for chromosome duplication such as the application of antimitotic chemical substances is necessary. Various doubling agents have been studied. They included colchicine, caffeine, nitrous oxide and the antymicrotubule herbicides: trifluralin, oryzalin and amiprophosmethyl. However, the most commonly used chemical agent is colchicine, because is easy to use, it is dissolved in water and the solution can be stored. Furthermore, colchicine can be applied during several stages of the technology of obtaining doubled haploid lines from isolated microspores to the regenerated plantlets and plant. The presented methods for chromosome doubling of oilseed rape haploids involve treatment of microspores in vitro just after their isolation from anthers, soaking roots of plantlets or whole plants in a colchicine solution, as well as after treatment of secondary auxiliary shoots of haploid plants. It seems that the freshly isolated microspores are best and very convenient to apply colchicine for their chromosomes doubling. The in vitro colchicine treatment of microspores does not cause any developmental delay; moreover it can improve the induction of embryogenesis. However, sometimes the number of chromosomes can be doublied in the plantlets by soaking their roots or by shoots of haploids immersed in a solution of colchicine. These methods are laborious, time consuming and result in sectorial ploidy chimeras. The two in vivo methods presented are used to duplicate chromosomes in haploid plants developed from different explants subjected to mutagenesis or transformation and in the case of plants obtained through interspecific crosses for the resynthesis of oilseed rape.

The purpose of this study is to evaluate the effects of in vitro colchicine treatment into the microspore culture and in vivo colchicine treatment of young plants and auxiliary haploid shoots with regard the efficiency of different diploidization methods of winter oilseed rape.

This work was partially supported by the Ministry of Agriculture and Rural Development of Poland. Project No 51. 
Poster no. 21

\title{
A preliminary study on DNA methylation patterns in sugar beet (Beta vulgaris L.) haploids
}

\author{
SANDRa CichorZ ${ }^{1 *}$, Matgorzata MaLicka ${ }^{1}$, Maria GoŚKA ${ }^{1}$ \\ Laboratory of Cytogenetic and Breeding Methodology, Department of Genetics and Breeding of Root Crops, \\ Plant Breeding and Acclimatization Institute - National Research Institute, Research Division in Bydgoszcz, Poland \\ *sandra.cichorz@interia.pl; s.cichorz@ihar.bydgoszcz.pl
}

\begin{abstract}
Sugar beet (Beta vulgaris $\mathrm{L}$.) is one of the most important food and sucrose-producing crops in the world, which provides $20 \%$ of the global sugar production. Additionally it serves as a significant plant for bioenergy in industrial and chemical feedstocks. Intensive breeding has led to the fact that sucrose accounts for up to $18 \%$ of plant fresh weight, most of which is especially concentrated in the taproot, where it accumulates in the vacuoles. Homozygous and genetically stable lines are necessary for crop improvement to maximize heterosis effect, but they are timeconsuming and labor-intensive to obtain through conventional methods of plant breeding. Haploid, and doubled haploid (DH) sugar beet plants which enable great time reduction, cannot be produced through androgenesis, the most favorable technique. In the above mentioned species an alternative method of unfertilized ovules and ovaries cultures is used.
\end{abstract}

During such a tissue culture, the plant cell reprogramming and acquisition of cellular totipotency represent highly stressful conditions whereby repression or activation of numerous genes as well as changes in the global genome organization occur. As a consequence, both epigenetic and genetic alterations are often induced and are collectively called somaclonal variation. This phenomenon may be useful in a plant breeding context for variety improvement, but it is undesirable in long-term genotype preservation. In recent years, the best known epigenetic process is DNA methylation, because it plays a crucial role in the control of spatial and temporal patterns of gene expression. A different kind of environmental stresses has been suggested to alter the DNA methylation pattern, which is necessary for a normal plant growth. This epigenetic mark in plants is not only species but also tissue, organ and age specific. During plant cell differentiation and proliferation processes, the levels and tissue distributions of DNA methylation may vary significantly.

Several methods have been developed to study either the global level of methylated cytosine or methylation at a specific gene sequence. Techniques such as high performance liquid chromatography (HPLC), bisulfites method and methylation sensitive amplification polymorphism (MSAP) have been developed. Among these, the use of restriction enzymes such as isoschizomers that share the same recognition sites but show differential sensitivity to DNA methylation at the restriction site represents an efficient technique for the detection of methylation changes. One method for determining the presence of anonymous methylated CCGG sites is methylation sensitive ISSR (MSISSR), where DNA is digested with HpaII and MspI and then analysed by ISSR PCR. Both enzymes will cut unmethylated CCGG sites, but HpaII will not cut if either the external or internal cytosine is fully methylated (i.e. methylated on both strands of DNA). In contrast, MspI will cut when the internal cytosine is methylated, although not if the external cytosine is fully or hemimethylated (i.e. methylated on a single DNA strand).

Therefore, the present study was carried out to assess the preliminary characteristics of the epigenetic changes related to genomic DNA methylation among the selected sugar beet haploid lines using MS-ISSR markers. 


\title{
Poster no. 22
}

\section{Rye (Secale cereale L.) androgenesis and the search for RAPD and ISSR markers related with the response in the anther culture}

\author{
Sylwia MikoŁajczyK *, Dorota Weigt, AgNieszka Tomkowiak, Zbigniew Broda \\ Department of Genetics and Plant Breeding, Faculty of Agronomy and Bioengineering, \\ Poznań University of Life Sciences, Poznań, Poland \\ *sylviam@up.poznan.pl
}

The capacity of haploids rye plants to regenerate through androgenesis in anther cultures is genetically controlled and it causes high diversification in the number of green and albino regenerants obtained. As it is a problem to break rye resistance to the induction of haploids, it is necessary to search for molecular markers of androgenetic capacity in the rye anther culture. The aim of the study was to assess the DNA polymorphism by means of RAPD and ISSR markers and to search for DNA markers related to androgenetic efficiency and regeneration of green plants in the anther culture of 53 rye genotypes.

The research was conducted on 13 rye population cultivars and 40 pre-breeding materials. The anther cultures of the rye genotypes were prepared according to the methodology described by Immonen and Tenhola-Roininen (2003) [1]. DNA was isolated from the donor plants by means of a Genomic Mini AX Plant kit. Next, the concentration and purity of the preparations were measured with a Nano Drop 2000 spectrophotometer. The DNA polymorphism was tested with 25 RAPD primers with 10 nucleotide sequences selected from studies by Bolibok et al. (2007) [3], Broda et al. (2008) [4], Ćwiklińska et al. (2010) [5] and Kiviharju et al. (2004) [6] and with 20 ISSR primers available in the studies by Bolibok et al. (2005) [2] and Hackauf and Wehling (2002) [7]. The RAPD-PCR and ISSR-PCR were carried out in a Professional Basic Gradient Biometra thermocycler. PCR products were separated in 1.5\% agarose gel. The electropherograms were documented with the BIORAD system. The molecular weights of the amplification products were calculated with the UVIBand v. 12.14 program. Genetic similarity was estimated with the DICE algorithm invented by Nei and Li (1979) [8]. Genetic similarity dendrograms were drawn with the DendroUPGMA program.

The tests conducted on 45 primers ( 25 primers for RAPD and 20 primers for ISSR) showed that there were more products obtained in the ISSR-PCR than in the RAPD-PCR. The genetic similarity values calculated according to the DNA polymorphism obtained by means of RAPD and ISSR markers ranged from $30 \%$ to $85 \%$. The dendrograms reflected the genetic similarity and origin of the 53 rye population cultivars and pre-breeding materials.

\section{References}

[1] Immonen S., Tenhola-Roininen. (2003) [In:] Doubled Haploid Production in Crop Plants. Ed. Maluszynski M. et al.: 141-149.

[2] Bolibok H., Rakoczy-Trojanowska M., Hromada A., Pietrzykowski R. (2005) Euphytica 146: 109-116.

[3] Bolibok H., Gruszczyńska A., Hromada-Judycka A., Rakoczy-Trojanowska M. (2007) Cel. Mol. Biol. Lett. 12(4): 523-535.

[4] Broda Z., Kurasiak-Popowska D., Kowalska A., Ćwiklińska A. (2008) Biul. IHAR 247: 65-71.

[5] Ćwiklińska A., Broda Z., Bocianowski J., Dobrzycka A. (2010) Acta Biol. Crac. Ser. Bot. 52(1) : 19-25.

[6] Kiviharju E., Laurila J., Lehtonen M., Tanhuanpää P., Manninen O. (2004) Agri. Food Sci. 13: 151-162.

[7] Hackauf B., Wehling P. (2002) Plant Breeding 121: 17-25.

[8] Nei M., Li W.-H. (1979) Proc. Natl. Acad. Sci. USA 76(10): 5269-5273.

\section{Acknowledgements}

Project is funded by Polish Ministry of Agriculture and Rural Development (HOR.hn.802.16.2017 Task No. 86). 


\title{
Genetic stability in yellow-flowered magnolias propagated in vitro
}

\author{
Agnieszka Wojtania *, Monika MarkieWicz \\ Department of Applied Biology, Research Institute of Horticulture, Skierniewice, Poland \\ *agnieszka.wojtania@inhort.pl
}

The genus Magnolia L. (Magnoliaceae) consists of over 250 species and its numerous hybrids and varieties are recommended for planting in gardens and urban green areas. Recently, magnolias with yellow flowers have been most popular. Due to difficulties in the propagation of magnolias by cutting or grafting, it is desirable to develop and optimize in vitro propagation methods. In plants produced by in vitro method, it is necessary to control the genetic identity of propagated microshoots at every stage of production. We use ISSR markers to analyze the genetic stability of Magnolia plants propagated in vitro. These markers have been widely used in the study of plant genetic structure, genetic diversity and genetic relationships.

Three magnolia hybrids were used for the study: Magnolia "Elizabeth" (M. acuminata $\times$ M. denudata), Magnolia "Yellow Bird" (M. acuminata var. subcordata $\times$ M. brooklynensis "Evamaria") and Magnolia "Butterflies" (M. acuminata "Fertile Myrtle" $\times$ M. denudata "Sawada's Cream"). Cultures were initiated from actively growing shoots of 3-year-old magnolia plants grown in a greenhouse. Initiation of the culture and multiplication of shoots were carried out on Murashige and Skoog (1962) medium (MS) modified by a reduction using $1 / 4$ dose of nitrogen salts and supplemented with $0.3 \mathrm{mg} / \mathrm{dm}^{3}$ benzylaminopurine (BAP). For rooting, shoots $>2 \mathrm{~cm}$ were cultured on MSmedium with the nitrogen salts reduced to half strength, supplemented with $6.0 \mathrm{mg} / \mathrm{dm}^{3}$ of indole-3-butyric acid (IBA).

In the present research, the genetic stability of magnolia shoots was evaluated in relation to the standards (mother plants). Genomic DNA was extracted from fresh leaves from in vitro shoots, using a DNeasy Plant Mini Kit (Qiagen) according to manufacturer's instructions. The genetic stability was assessed using an inter simple sequences repeat analysis (ISSR). 18 ISSR primers (UBC Primer Set, University of British Columbia) were used for analysis according with Wolfe (2005) [1]. Five primers that differentiate tested cultivars were used to assessed the similarity between ISSR profiles corresponding standard plant respectively microshoots regenerated in vitro. ISSR primers generated a total of 53 bands that were monomorphic for all plants analyzed, including the standard plants. The number of bands for each primer varied between 9 (UBC827), 7 (UBC828), 15 (UBC834), 12 (UBC848) and 10 (UBC855), with an average of 10.6 bands per primer. Regarding the size of amplified DNA fragments, it varied between $200 \mathrm{bp}$ and $1250 \mathrm{bp}$.

Based on the ISSR data, no genetic variation was detected for shoots. At this stage of study, there we no changes in the number and distribution in DNA gel bands obtained after the amplification of ISSR markers in regenerated magnolia shoots.

\section{Acknowledgements}

This work was supported by the Ministry of Agriculture and Rural Development - Project no. 10.1.3: Optimization of in vitro propagation technology of some genotypes from the genus Vaccinium, Syringa, Magnolia, Helleborus, Paeonia.

\section{References}

[1] Wolfe A.D. (2005) Meth. Enzymol. 395: 134-144. 


\title{
Poster no. 24
}

\section{Evaluation of the possibility of adventitious regeneration of gooseberry (Ribes grossularia L.) in vitro}

\author{
ANgelika NieWiadomska-WnUK *, Danuta Kucharska \\ Research Institute of Horticulture, Skierniewice, Poland \\ * angelika.niewiadomska_wnuk@inhort.pl
}

Knowledge of the adventitious organogenesis procedure is a prerequisite for the use of polyploidisation, mutagenesis, transformation in breeding programms or in the selection of specific factors for the in vitro conditions. The ability to form of adventitious shoots depends on many factors, including the genotype, the type of explant used, the state of the donor cultures, the type and concentration of growth regulators and other constituents of the medium. So far, the published literature has not reported on the adventitious regeneration of Ribes grossularia $\mathrm{L}$. The objective of the work was to examine the possibility of the regeneration in vitro of adventitious shoots of five genotypes of gooseberry. The study was conducted on three cultivars "Pax", "Invicta" and "Hinsel", and on two breeding clones 86 and 2/33. Leaf explants were incubated on the induction media containing tidiazuron (TDZ) in concentrations of 0.25 or $0.5 \mathrm{mg} / \mathrm{dm}^{3}$, then they were transferred to regeneration media with the following composition: $0.01 \mathrm{mg} / \mathrm{dm}^{3}$ IAA with the addition of $0.1 \mathrm{mg} / \mathrm{dm}^{3} \mathrm{TDZ}$ or $0.5 \mathrm{mg} / \mathrm{dm}^{3}$ meta-topolin (mT). In vitro donor cultures were conditioned on the medium with the addition of $1.0 \mathrm{mg} / \mathrm{dm}^{3} \mathrm{mT}$ or $0.2 \mathrm{mg} / \mathrm{dm}^{3} \mathrm{TDZ}$ for 30 days. The studies showed that the media used did not have any significant or uneven influence on the efficiency of an adventitious regeneration. The ability of adventitious regeneration depended mainly on the genotype. Three gooseberry cultivars were tested and regardless of the factors used, they showed very little capacity for adventitious regeneration. More adventitious shoots produced clones 86 and 2/33. More adventitious shoots were formed after 14-days induction than after 7 days. Conditioning of donor cultures on the medium with TDZ did not increase of the frequency of adventitious shoots compared to the control medium with $\mathrm{mT}$. The regeneration medium with TDZ stimulated callus growth in all tested genotypes. There are many reports on the procedures for an effective adventitious regeneration of fruit plants such as: Rubus [2], Malus [3], Vaccinium species [4]. The previous literature data made no mention of the adventitious regeneration of Ribes grossularia L. Raghu et al. (2006) [5] reported an adventitious organogenesis from leaf explants at Embelia ribes Burm and Arena and Pastur (1997) [1] at Ribes magellanicum. They carried out a successful regeneration on media supplemented with BAP and NAA but reported big problems with the callus and the browning of explants. Our current two-year studies on gooseberry adventitious regeneration allow to eliminate or reduce the factors inhibiting this process. This species, belonging to the adventitious organogenesis that is difficult to initiate, requires more work to develop an efficient procedure of adventitious regeneration.

\section{References}

[1] Arena M.E., Martinez Pastur G.J. (1995) Poiret. Sci. Hort. 62: 139-144.

[2] Fiola J.A., Hassan M.A., Swartz H.J., Bors R.H., McNicols R. (1990) Plant Cell Tiss. Org. Cult. 20: 223-228.

[3] James D.J., Passey A., Rugini E. (1988) J. Plant Physiol. 132: 148-154.

[4] Meiners J., Schwab M., Szankowski I. (2007) Plant Cell Tiss. Org. Cult. 89(2-3): 169-176.

[5] Raghu A.V., Geetha S.P., Balachandran G.M.I., Ravindran P.N. (2006) J. For. Res. 11: 57-50. 


\title{
Assessment of the genetic stability of Taraxacum pieninicum microcuttings after long-term slow growth storage using molecular markers
}

\author{
Monika KamińsKa *, Alina Trejgell \\ Department of Plant Physiology and Biotechnology, Mikołaj Kopernik University in Toruń, Poland \\ *moni.ka.minskaa@gmail.com
}

Plant conservation is associated with the increasingly noticeable adverse effects of environmental changes resulting in the extinction of plant species. Plant conservation programs focus mainly on seed banks. However, seeds of some species lose their vitality quickly or they are not readily available [2]. Tissue culture in vitro resolves this problem by a synthetic seeds technique. Encapsulation of embryogenic or meristematic tissue is a cost-efficient and simplified option for plant germplasm conservation [3]. However, the quality control of micropropagated plant material has to be verified before the introduction of any species into the natural environment. Different markers and techniques are available for the assessment of the genetic stability of plants propagated both in vitro and in vivo. PCR-based methods such as RAPD (Random Amplification of Polymorphic DNA) and ISSR (Inter Simple Sequence Repeat) have become an efficient and common tool in establishing the genetic stability because no prior sequence information is needed. However, these methods have various limitations which have been largely resolved by genetargeted markers such as Start Codon Targeted Polymorphism (SCoT). SCoT is based on the short conserved region flanking the ATG start codon [1].

The aim of the present study was to test the genetic homogeneity of in vitro derived and stored plants of Taraxacum pieninicum using RAPD, ISSR and SCoT techniques.

The plant material was a $T$. pieninicum microshoots obtained from axillary buds on shoot tips. The shoots were multiplied according to the protocol described by Trejgell et al. [4]. Synthetic seeds were obtained by polymerization of $3 \%$ sodium alginate in $100 \mathrm{mM} \mathrm{CaCl} 2 \cdot 2 \mathrm{H}_{2} \mathrm{O}$ and stored under reduced light or in the dark at $4{ }^{\circ} \mathrm{C}$ for 12 months. DNA was isolated from plants acclimatized after storage using a modified Doyle and Doyle (1987) method with an extraction buffer containing CTAB. Plants germinated from natural seeds and those grown in pots were used as a control. After primer screening of control plants, 21, 19 and 12 starters were used in the RAPD, ISSR and SCoT analyses, respectively.

Each tested primer produced clear and scorable amplification products. The total number of bands was 67,51 and 83 for RAPD, ISSR, and SCoT technique, respectively. All the RAPD, ISSR and SCoT profiles from plantlets were monomorphic and comparable to the control plants. Based on the results, it can be concluded that in vitro micropropagation and synseeds storage of $T$. pieninicum do not generate genetic changes on the basis of the primers used. Therefore, plants from an in vitro culture can be used for the reintroduction of this species into the natural habitat.

\section{References}

[1] Collard B.C.Y., Mackill D.J. (2009) Plant Mol. Biol. Rep. 27: 86-93.

[2] Fay M.F. (1992) In Vitro Cell. Dev. Biol. Plant 28(1): 1-4.

[3] Ray A., Bhattacharya S. (2008) South Afr. J. Bot. 74(4): 776-779.

[4] Trejgell A., Chernetskyy M., Podlasiak J., Tretyn A. (2013) Acta Biol. Cracov. Ser. Bot. 55: 73-79. 


\title{
Poster no. 26
}

\section{Prospects and limitations of in vitro propagation of rare, native plant species from Ranunculaceae family}

\author{
DAWID KocoT \\ Institute of Plant Biology and Biotechnology, University of Agriculture in Cracow, Poland \\ *dawid_2608@vp.pl
}

Plant tissue culture techniques give the opportunity to obtain a large number of plants within a short period of time starting with a small amount of the maternal material. Therefore, their use for the propagation of rare plants is frequent, particularly when the number of mother plants is small and their regenerative potential is limited for various reasons. It is also an alternative method of reproduction of these species, which due to their decorative qualities are the object of commercial interest. Such rare native species include Ranunculus illyricus L. and Aconitum bucoviniense Zapał. listed in the Red book, as well as legally protected Pulsatilla pratensis (L.) Mill. The choice of an appropriate explant to initiate cultures is crucial for experiment success. The preferred way to start cultivation of rare plants is to sow decontaminated seeds onto a medium. Tetrazoline staining revealed that all three species had developed viable seeds (Ranunculus - 93\%, Aconitum - 100\%, Pulsatilla - 90\%), however, only the seeds of $P$. pratensis germinated in post-harvest sowing both ex vitro and in vitro. The application of dormancy breaking treatment did not improve the ability of Ranunculus seeds to germinate.

The isolation of the apical buds is an alternative method of initiating cultures, but all three species have hairs that hinder proper disinfection. The isolated buds of $R$. illyricus were easy to grow on kinetin and NAA supplemented media but infections appeared in the next subculture, gradually eliminating initially disinfected buds by the end of the third passage.

Callus cultures of $R$. illyricus were induced on fragments of leaves, petioles, rhizomes and flower receptacle. The effectiveness of disinfection ranged from 28.5 to $100 \%$ depending on the type of explant. Well-proliferating callus was obtained on fragments of rhizomes cultivated on a medium with the addition of kinetin and picloram.

Callus of $A$. bucoviniense was induced on leaf explants and petioles. The efficiency of decontamination was about $42 \%$, and callus growth was recorded on the media supplemented with kinetin and picloram. It was shown for P. pratensis that the quality of the mother plant can be the decisive factor in the process of micropropagation. The shoots cultivated on media with IAA and $2 \mathrm{iP}$ did not multiply when mother plants originated from the collection, whereas in the case of seeds representing natural populations, the multiplication coefficient reached about 3-4. However, the obtained shoots rooted with great difficulty, which makes a challenge to obtain plants for acclimatization.

Each of the discussed species is a valuable piece of native nature and there is a need to develop effective methods of their multiplication in tissue cultures, however, the problems observed at various stages of cultivation place them within of the most difficult species for micropropagation. 
Poster no. 27

\title{
Identification of the apical cells of the Thelypteris palustris Schott gametophyte in an in vitro development
}

\author{
KORNEl M. MichalaK, ElŻBieta ZenKTELER \\ Department of General Botany, Faculty of Biology, Adam Mickiewicz University in Poznań, Poland
}

Presently, scientific knowledge of the fern gametophyte development remains incomplete. Of particular interest is the role of apical cell as a proliferative center and the regulatory site of the gametophyte. Germinating filament is an interesting model for investigation of the asymmetric division and differentiation of the apical cell. The aim of the study was to determine whether the development of gametophytes depends on the one type of the apical cell or on a variety of forms of apical initials.

The Marsh Fern (Thelypteris palustris) is native to the eastern part of North America and Eurasia. It grows mainly in marshy wet places of a wide $\mathrm{pH}$ range, in banks of water reservoirs or at meadows with a high level of groundwater. T. palustris is a perennial plant with thin, black, plagiotropous, long-creeping rhizomes, forming colonies. Leaves reach to $50-80 \mathrm{~cm}$ of length. The optimum spores dissemination stretches from August to early October. The spores are bilateral, ranging from $20 \times 30$ and $40 \times 60 \mu \mathrm{m}$. Granulose or spinulose perine is adherent to exine. Spore germination of $T$. palustris is of the Vittaria-type begins with the protrusion of small caulonema and large chloronema cells. The rhizoid elongates by tip growth, the domed-shape apical cell divides to form a primary (3-5 cells) filament. The periclinal division of their subterminal cell gives rise to the spatulate plate formation (twodimensional growth). The prothallial development is of the Drynaria-type [1]. T. palustris gametophyte has a small, reniform, hairy, long lived thallus, cordate at maturity, with a wide cushion, overlapping wings and a sex organ of the advanced type. In a recent study we confirmed that the plane dividing the apical cell was early replaced by the cuneate apical cell (with two cutting faces) and their merophytes produced an asymmetrical cordate gametophyte with a folded margin. A few secondary apical cells with unicellular papillate secretory hairs appear at the margin of the gametophyte, which sometimes develop small vegetative prothalli. At the stage of the cushion development the prismatic apical cell was found in the mass of small, uniform hexagonal cells (three-dimensional growth). The cell (with three cutting faces) may be of an isodiametric shape. At the last stage of development associated with the sexual organ formation (antheridia appeared before the archegonia) secondary apical cells appear first. The sequential appearance of four types of apical cells and apical initials in a cell lineage determined the development of a complete $T$. palustris gametophyte. According to formative capacities of apical cells the gametophyte passes through three shapes i.e. filamentous, spatulated and heart-shaped.

Procedures: Spores of Thelypteris palustris were collected from the field of the Turek district (Wielkopolska Region). Sporophylls were collected from a few mature individuals in September. The leaves were dried between sheets of paper, and the spores, after their release from sporangiums, were stored at $4{ }^{\circ} \mathrm{C}$ until used. After surface disinfection ( $1 \mathrm{sec}$ in $70 \%$ ethanol, $3 \times \mathrm{SDW}$ ) spores were sown and germinated in $1 / 2 \mathrm{MS}$ medium solidified with $6 \mathrm{~g} \cdot \mathrm{l}^{-1}$ agar. After imbibition in the dark for $12 \mathrm{~h}$ spores were illuminated (photon flux of $30-40 \mu \mathrm{mol} \mathrm{m}^{-2} \mathrm{~s}^{-1}$ ). The cultures were maintained at $22^{\circ} \mathrm{C}$ with a relative humidity of $45 \%$. The spores at different germination and developmental stages were examined under light and fluorescent microscopes.

\section{References}

[1] Nayar B.K., Kaur S. (1971) Bot. Rev. 37(3): 295-382.

[2] Atkinson L.R. (1973) Bot. J .Linn. Soc. 66: 23-36.

[3] Sen T. (1981) Blumea 27: 239-254. 


\title{
The role of endogenous auxins and cytokinins in the process of winter wheat vernalization - impact of zearalenone in in vitro conditions
}

\author{
Michą DZiURKa *, Kinga DZiURKa, AgnieszKa OstrowsKa, \\ anna Janeczko, Franciszek Dubert, Jolanta Biesaga-KościelniaK
}

The Franciszek Górski Institute of Plant Physiology, Polish Academy of Sciences, Kraków, Poland

*m.dziurka@ifr-pan.edu.pl

Plants have evolved many systems to sense their environment and to modify their growth and development. An example of this is vernalization, the process by which flowering is promoted as plants sense the exposure to cold temperatures. The physiology of vernalization in a wide range of species has been intensively studied for many decades, but the process is still not fully understood. A fungal secondary metabolite, zearalenone (ZEN), showed high activity that was achieved thanks to the shortening period of flowering during the vernalization of winter wheat [1]. The aim of the study was to verify the hypothesis that the effect of zearalenone leading to a break of the flowering blockade in winter wheat establishes a specific hormone balance during vernalization. The experiments were carried out in vitro. The isolated winter wheat embryos of Kobiera cultivar were grown on Murashige and Skoog medium (MS) with ZEN and MS medium without ZEN (control). Both were subjected to a shortened vernalization time $\left(5^{\circ} \mathrm{C}\right.$ for 0 , $3,6,9,12$ and 15 days), followed by a 10 -day acclimation at $10^{\circ} \mathrm{C}$, and afterwards transferred to an open vegetation tunnel. After 120 days of vegetation, the degree of apical meristem development was determined according to the 12-degree scale of the wheat organogenesis [3]. Auxin and cytokinins were monitored by UHPLC-MS/MS [2]. Samples were collected after $0,3,6,9,12$ and 15 days of vernalization at $5^{\circ} \mathrm{C}$ fallowed by a 10 -day acclimation at $10^{\circ} \mathrm{C}(9 / 15 \mathrm{~h}$ photoperiod $(\mathrm{d} / \mathrm{n})$. In the control plants population, only $10 \%$ achieved a generative developmental phase. In contrast, plants vernalized in the presence of ZEN reached the generative phase in $80 \%$ already after 6 days of cooling, and after 15 days all plants were generative. In the shoot growth apex, a tendency for a gradual accumulation of both forms of cytokinins (active and inactivated) was observed, proportionally to the time of vernalization. Concurrently, the auxin concentration (both biologically active and inactivated forms) has decreased, which in turn caused a corresponding increase in the cytokinin to auxin ratio. However, the intensity of these changes was too low to allow the transition of most plants to the generative phase. The introduction of ZEN into the medium caused the aleviation of the cytokinin concentration (active and inactivated) to be raised twice in plants subjected to 0 and 15 days of vernalization. In plants vernalized for 6 days the content of the deactivated forms of auxins significantly increased. Simultaneously, the use of ZEN drastically reduced the accumulation of auxins, both in the active and in the inactivated form, and the cytokinin/auxin balance was gradually shifted in favor of cytokinins. An in vitro culture could be a useful tool for researching the regulation of the vernalization processes. On the basis of the obtained results, it can be assumed that the action of ZEN as a promoter of the generative winter plant induction consists of the specific determination of mutual proportions of cytokinins and auxins, i.e. the inhibition of auxin accumulation and the stimulation of cytokinin concentration.

\section{Acknowledgments}

This research was supported by the funds: 5457/E-189/S/2016 and 2/5457/E-189/M/2015 (Polish Ministry of Science and Higher Education).

\section{References}

[1] Biesaga-Kościelniak J., Filek M. (2010) [In:] Sustainable agriculture reviews 3. Sociology, organic farming, climate change and soil science. Ed. E. Lichtfouse.Berlin, Springer: 419-435.

[2] Dziurka M., Janeczko A., Juhasz C. et al. (2016) Plant Physiol. Biochem. 109: 355-364.

[3] Kuperman F. (1965) Biologiczne obserwacje kontrolne w rolnictwie. PWRiL, Warszawa. 


\title{
The effect of meta-topolin and thidiazuron on shoot regeneration in hemp (Cannabis sativa L.)
}

\author{
Mariola Dreger *, Grażyna MańKowska, Milena Szalata \\ Department of Biotechnology, Institute of Natural Fibres and Medicinal Plants, Poznań, Poland \\ *mariola.dreger@iwnirz.pl
}

Hemp (Cannabis sativa L.) and cannabinoids enjoy a considerable interest within the pharmaceutical industry. Approximately 100 phytocannabinoids have been identified in hemp plants, showing a potential therapeutic activity. The most abundant cannabinoids are $\Delta$ 9-tetrahydrocannabinol ( $\triangle 9$-THC) and cannabidiol (CBD). A low - THC cultivars with a high $\mathrm{CBD}$ content have the most promising medical potential. However, the cannabinoid content in plants is variable and depends on both genetic and environmental factors. Therefore, a simple and efficient regenerative system is needed for elite clones propagation. Genotype dependent response of explants and a low regeneration frequency are the main limitations in hemp in vitro propagation [2]. The aim of this study was to investigate the effect of meta-topolin (mT) and thidiazuron (TDZ) on shoot regeneration from epicotyls and hypocotyls explants of fibre type of $C$. sativa.

Seeds were sterilized in 70\% ethanol and in commercial bleach (ACE $®)$ and then washed 3-4 times in autoclaved water. Sterilized seeds were placed on Petri dishes containing MS [1] medium without PGR (Plant Growth Regulators) for germination. Shoot induction was initiated from hypocotyl and epicotyl explants $(0.5-1.0 \mathrm{~cm})$. An MS medium supplemented with various concentrations $\left(0.0-1.0 \mathrm{mg} / \mathrm{dm}^{3}\right)$ of $\mathrm{mT}$ and TDZ was used. Cultures were grown under a $16 \mathrm{~h}$ photoperiod, at a light intensity of $50-60 \mu \mathrm{mol} / \mathrm{m}^{2} \mathrm{~s}$, provided by Osram fluora L58 W/77 lamps, at $25 \pm 1^{\circ} \mathrm{C}$ (standard conditions). After four weeks of incubation explants formed new shoots. Epicotyls were the most responsive explants for shoot induction. Hypocotyls occasionally (3-16\%) generated new shoots on a medium with TDZ. Epicotyl explants produced adventitious shoots with the highest induction frequency (100\% and 94\%) on a medium supplemented with TDZ $\left(1.0 \mathrm{mg} / \mathrm{dm}^{3}\right)$ and with a combination of $\mathrm{mT}\left(0.5 \mathrm{mg} / \mathrm{dm}^{3}\right)$ and TDZ $\left(0.5 \mathrm{mg} / \mathrm{dm}^{3}\right)$. The maximal average number of shoots per explant $(3.9 \pm 1.12 \mathrm{SD})$ was noted for a medium with $1.0 \mathrm{mg} / \mathrm{dm}^{3}$ of TDZ. A negative effect of TDZ (hyperhydricity of explants) at a high concentration was observed. The regenerated shoots were isolated and rooted on $1 / 2 \mathrm{MS}$ medium supplemented with IAA $\left(0.5 \mathrm{mg} / \mathrm{dm}^{3}\right)$. The in vitro protocol may be utilized for multiplication and conservation of elite clones of $C$. sativa.

\section{Acknowledgement}

The work was financed by the Ministry of Agriculture and Rural Development (RM 171/2017).

\section{References}

[1] Murashige T., Skoog F. (1962) Physiol. Plant 15: 473-497.

[2] Wróbel T., Dreger M., Wielgus K., Słomski R. (2018) Biotechnol. Lett. 40(3): 445-454. 


\title{
Poster no. 30
}

\section{Effect of purine-type cytokinins on shoot proliferation of Salvia bulleyana}

\author{
MONIKA KOWALSKA *, IZABELA GRZEgORCZYK-KAROLAK \\ Department of Biology and Pharmaceutical Botany, Medical University of Łódź, Poland \\ *monikakowalska170@wp.pl
}

The genus Salvia, the largest in the family Lamiaceae counting almost 1000 species, is widely used in perfumery, food industry and medicine. The habitat of about 100 species of these 1000 is Eastern Asia. Salvia bulleyana Diels. is a folk medicinal plant native to the Chinese Yunnan Province. This species has been used in traditional Chinese medicine and referred to as Danshen (S. miltiorrhiza roots). S. bulleyana has a therapeutic potential in vascular and heart diseases such as stroke, angina pectoris or myocardial infarction. It has been used in the treatment of liver fibrosis, osteoporosis, inflammatory diseases and insomnia [1,2]. The pharmacological properties of the plants are mainly due to the presence of polyphenolic acids such as caffeic acid, rosmarinic acid, salvianolic acids and ditepenoids such as cryptotanshinone, dihydrotanshinone, tanshinone I and IIA $[2,3]$.

The aim of our study was to establish a relatively fast system for the propagation of $S$. bulleyana, which is a rare species. In vitro culture techniques offer new opportunities for obtaining a large amount of high-value plant material. The study evaluates the effects of various purin-derivative cytokinins on S. bulleyana shoot proliferation. The shoot tips from five-week-old shoots growing in vitro were used as explants in the experiment. They were incubated on MS (Murashige and Skoog) [4] agar medium supplemented with IAA (indole-3-acetic acid) at a concentration of 0.1 or $0.5 \mathrm{mg} / \mathrm{dm}^{3}$ and various cytokinins (meta-topoline - M-Top, 6-benzylaminopurine riboside - RBAP, N-benzyl-9-(2tetrahydropyranyl)-adenine - BPA) at three concentrations $\left(0.5,1\right.$ or $\left.2 \mathrm{mg} / \mathrm{dm}^{3}\right)$.

Our results demonstrate that the type of cytokinin and its concentration as well as auxin concentration significantly influence the regeneration potential of $S$. bulleyana shoots. The highest multiplication rate was obtained when $0.1 \mathrm{mg} / \mathrm{dm}^{3}$ IAA and BPA at a concentration of $2 \mathrm{mg} / \mathrm{dm}^{3}$ was used. Under those conditions after 5 weeks, $100 \%$ shoot tips formed buds or shoots and almost 7 buds/shoots per explant were obtained. On the other hand, the highest biomass of the culture was found for shoots growing on a medium supplemented with m-Top at a concentration $1 \mathrm{mg} / \mathrm{dm}^{3}$; it was $1.2 \mathrm{~g}$ of dry weight and $0.17 \mathrm{~g}$ of fresh weight. In this case we obtained an average of 5 new buds/shoots on explant. Additionally, it was observed that increasing the concentration of cytokinin in the medium inhibited the shoot elongation, meanwhile in most cases, raising auxin (IAA) concentration (from 0.1 to $0.5 \mathrm{mg} / \mathrm{dm}^{3}$ ) reduced the percentage of explants giving response in the form of bud/shoot formation.

\section{References}

[1] Topcu G.J. (2006) Nat. Prod. 69: 482-487.

[2] Li M.H., Chen J., Peng Y., Wu Q., Xiao P.G. (2008) J. Ethnopharmacol. 120: 419-426.

[3] Li M.H., Peng Y., Xiao P. (2010) J. Syst. Evol. 48: 118-122.

[4] Murashige T., Skoog F. (1962) Physiol. Plant. 15: 473-479. 
Poster no. 31

\title{
The influence of the type of cytokinin on the initiation and stabilization of highbush blueberry Vaccinium corymbosum $\mathrm{L}$. in in vitro
}

\author{
Danuta Kucharska*, StanisŁaw Pluta \\ Research Institute of Horticulture, Skierniewice, Poland \\ *danuta.kucharska@inhort.pl
}

Plant propagation in vitro is one of the methods of clonal propagation widely used in the production of high quality nursery plants, in breeding programs and in a mass propagation of species difficult to reproduce in a traditional way, as for example highbush blueberry (Vaccinium corymbosum L.). The first reports on micropropagation of highbush blueberry were published by Zimmerman and Broome in 1980 [6], Orlikowska in 1986 [4] and Chandler and Draper in 1986 [1]. Since then, several authors have reported on the progress in the in vitro multiplication of the plants from genus Vaccinium spp. They underlined the role of the type and concentration of growth regulators in the initiation medium. In most studies zeatin or $2 \mathrm{iP}$ with auxin IAA or IBA were used.

In our study, the experimental material consisted of 25 genotypes (seedlings) of highbush blueberry, selected in the Laboratory of Genetics and Plant Breeding of Fruit Crops Research Institute of Horticulture in Skierniewice. Initiation and stabilization of the in vitro cultures processes were initially carried out on a medium containing $5 \mathrm{mg} / \mathrm{dm}^{3} 2 \mathrm{iP}$. The percentage of survived initial explants excluding the infected ones was no more than $48 \%$. The losses of explants caused by dieback occurred also during the stabilization period and reached to $69 \%$. In one of the seedling over $90 \%$ of explants died. In the second year of study, the initial and stability steps were carried out on the medium containing $1 \mathrm{mg} / \mathrm{dm}^{3}$ zeatin. The results obtained showed a significant reduction in the number of dying explants. On average, over $31 \%$ of explants survived initiation and stabilization periods on the medium with zeatin and only $12 \%$ on the medium with $2 \mathrm{iP}$. As reported by Debnath [2], cytokinin $2 \mathrm{iP}$ shows a phytotoxic effect especially in the stabilization phase of blueberry culture, causing browning and dying of explants. This may explain a very large percentage of losses in our experiments. Reed and Abdelnour-Esquivel [5] and Gonzalez et al. [3] reported greater effectiveness of zeatin both in the initiation and propagation stages of blueberry, which was also confirmed in our studies.

\section{References}

[1] Chandler C.K., Draper A. D. (1986) HortSci. 21: 1065-1066.

[2] Debnath S.C. (2007) Int. J. Fruit Sci. 6: 47-71.

[3] Gonzalez M.V., Lopez M., Valdes A.E., Ordas R.J. (2000) Ann. Appl. Biol. 137: 73-78.

[4] Orlikowska T. (1986) Fruit Sci. Rep. 13: 105-115.

[5] Reed B.M., Abdelnour-Esquivel A. (1991) HortSci. 26: 1320-1322.

[6] Zimmerman R.H., Broome O.C. (1980) [In:] Proceedings of the Conference on Nursery Production of Fruit Plants Through Tissue Culture - Applications and Feasibility, p. 44-47. USDA-SEA, Agr. Res. Results ARR-NE-11. 


\title{
Poster no. 32
}

\section{The effect of plant growth regulators on the regeneration of selected Polish varieties of flax (Linum usitatissimum L.)}

\author{
Grażyna Mankowska*, Milena Szalata, Karolina WielguS \\ Department of Biotechnology, Institute of Natural Fibers and Medicinal Plants, Poznań, Poland \\ *grazyna.mankowska@iwnirz.pl
}

Flax (Linum usitatissimum L.) is considered as one of the oldest fiber plants grown on all continents and has a wide range of applications in industry. Thanks to the popularity and a wide application of flax, the demand for plant material is constantly high. Plant biotechnology, in particular micropropagation, where flax is used as a model plant, plays an important role in improving traditional cultivation. The micropropagation process allows obtaining selected plant varieties with a high multiplication rate.

The aim of this work was to obtain sterile seedlings of flax (Linum usitatissimum L.) and to investigate the influence of individual plant growth regulators on the induction of adventitious shoots from hypocotyl explants. Two varieties of flax were examined - fibrous Nike and oil Bukoz, which were grown at the Institute of Natural Fibers and Medicinal Plants in Poznan. The experiment was established in three replications for each of Nike and Bukoz varieties.

In the experiment, 4084 seeds of Nike variety and 3949 seeds of Bukoz were placed on the medium. Shoot cultures derived from flax hypocotyls (5 millimeter fragments) which were obtained from 7-day old sterile seedlings. The explants were placed on MS medium with various concentrations of auxins and cytokinins $\left(1 \mathrm{mg} / \mathrm{dm}^{3} \mathrm{BAP}\right.$ and $0.02,0.2 \mathrm{mg} / \mathrm{dm}^{3}$ NAA respectively) and only with the addition of cytokinin $\left(0.5 ; 1 ; 2 \mathrm{mg} / \mathrm{dm}^{3} \mathrm{TDZ}\right)$. During the experiment, observations were conducted after 3 and 6 weeks from placing the explants on the medium. After 3 weeks, $98 \%$ of regenerating Nike variety on MS medium (control) were noted. Bukoz variety behaved differently, the best effect was observed on the medium with $1 \mathrm{mg} / \mathrm{dm}^{3}$ BAP and $0.02 \mathrm{mg} / \mathrm{dm}^{3} \mathrm{NAA}$ ), almost 95\% (SD 6.6) of regenerating explants were observed. In the case of a medium supplemented with TDZ in three concentrations, the results of Nike variety oscillated at the level of 20\% regenerating explants and Bukoz variety at the level of 35\%.

After 6 weeks, the induction of adventitious shoots from the hypocotyls of both Nike and Bukoz flax strains was more effective when using NAA (auxin concentration $0.2 \mathrm{mg} / \mathrm{dm}^{3}$ and $0.02 \mathrm{mg} / \mathrm{dm}^{3}$ ) in combination with BAP than the cytokine TDZ itself.

The highest average number of shoots 12.4 (SD 4.1) was obtained with a correspondingly high multiplication rate of 1.9 (SD 1.03) in Bukoz variety on the medium with the addition of BAP $1 \mathrm{mg} / \mathrm{dm}^{3}$ and NAA $0.2 \mathrm{mg} / \mathrm{dm}^{3}$. Similar results were observed on the control medium, the multiplication rate oscillated at 1.9 (SD 0.9) with the average number of shoots 11.5 (SD 4.7).

Nike variety was characterized by the highest multiplication rate of 3.4 (SD 2.7) and the number of shoots 12.0 (SD 8.6) on medium with BAP $1 \mathrm{mg} / \mathrm{dm}^{3}$ and NAA $0.02 \mathrm{mg} / \mathrm{dm}^{3}$ in comparison with MS (control) medium, on which the multiplication rate 1.6 (SD 0.9) and the number of shoots 8.9 (SD 4.1) were obtained. The lower concentration of auxins than cytokinins in the medium influenced the intensive growth of shoots, the best effect was obtained for Nike variety at the concentration of $0.02 \mathrm{mg} / \mathrm{dm}^{3}$ NAA.

The highest percentage of hypocotyl explants that produced shoots (69.7\%) was observed in Bukoz variety on the medium with the addition of $1 \mathrm{mg} / \mathrm{dm}^{3} \mathrm{BAP}$ and $0.02 \mathrm{mg} / \mathrm{dm}^{3} \mathrm{NAA}$. A high percentage of explants (63\%) of Nike variety produced stems on MS medium (control). A comparable percentage of explants for both varieties (52\% Nike, $55 \%$ Bukoz) was observed on the medium with the addition of $1 \mathrm{mg} / \mathrm{dm}^{3} \mathrm{BAP}$ and $0.2 \mathrm{mg} / \mathrm{dm}^{3} \mathrm{NAA}$. Explants growing on media with the addition of TDZ did not show the potential to form adventitious shoots.

\section{Acknowledgement}

The work was financed by Ministry of Agriculture and Rural Development (RM 171/2017). 
Poster no. 33

\title{
The regenerative abilities of Codonopsis pilosula (Franch.) Nannf. explants
}

\author{
JoANna MaKowCZyŃSKa *, EWa SKata, HaLina WysokińsKa \\ Department of Biology and Pharmaceutical Botany, Medical University of Lodz, Poland \\ * joanna.makowczynska@umed.lodz.pl
}

Codonopsis pilosula (Franch.) Nannf. is a plant belonging to the Campanulaceae family. This species is known as poor mans ginseng. $C$. pilosula is a twining perennial growing up $2 \mathrm{~m}$. This species is found in the natural habitat of Asian meadows, scrub and forests. Several species of the genus Codonopsis and among them is $C$. pilosula are widely used in traditional medicine because they have numerous pharmacological properties. In the Chinense Pharmacopoeia, the raw material is Codonopsis Radix which includes roots of different species of the genus $\mathrm{Co}$ donopsis such as $C$. pilosula, as well as $C$. pilosula var. modesta and $C$. tansheng [1]. In China this raw material is known as "Dangshen", and in Japan as "Tojin". Codonopsis Radix has been used in Chinense medicine for replenishing vital energy deficiency, improving gastrointerstinal function, strengthening the immune system [2]. The pharmacological properties of $C$. pilosula roots are associated with the presence of various secondary metabolites such as polysaccharides, triterpenes, phenylpropanoids, alkaloids and polyacetylenes [3].

It is important that raw materials of $C$. pilosula are collected not only from the natural habitat, which requires destruction of the whole plant. Biotechnological techniques may be an alternative to the standard cultivation. For this reason, the regenerative abilities of the $C$. pilosula explants were tested.

For the regeneration of $C$. pilosula, shoot tips and nodes were used as explants. In order to obtain adventitious buds, cotyledons, leaves, stems, hypocotyls and roots derived from 5-week-old seedlings were used. The explants were cultured on Murashige and Skoog (MS) medium supplemented with indole-3-acetic acid (IAA) $\left(0.1 \mathrm{mg} / \mathrm{dm}^{3}\right)$ and one of the cytokinins. The influence of 6-benzyladenine (BA), kinetin (6-furfurylaminopurine-KIN), zeatin (ZEA) at selected concentrations $\left(0.1 ; 0.5 ; 1.0 ; 3.0 \mathrm{mg} / \mathrm{dm}^{3}\right.$ and for ZEA addition $\left.5 \mathrm{mg} / \mathrm{dm}^{3}\right)$ was investigated. The cultures were grown for 6 weeks. The MS medium supplemented with IAA $\left(0.1 \mathrm{mg} / \mathrm{dm}^{3}\right)$ and BA $\left(0.5 \mathrm{mg} / \mathrm{dm}^{3}\right)$ was the best for the shoots multiplication - about 14 axillary buds and shoots per one shoot tip. A similar number of micropropagated structures was obtained from a single node on MS medium in the presence of $0.5 \mathrm{mg} / \mathrm{dm}^{3}$ of cytokinin $\mathrm{KIN}$ and $0.1 \mathrm{mg} / \mathrm{dm}^{3}$ IAA. The ability to rooting of shoots derived from nodal explants on MS medium without growth regulators or with addition of auxin was also checked. The formation of roots depended on the presence of auxins, IAA or IBA and on their concentration $\left(0.1 ; 0.5 ; 1.0 ; 2.0 \mathrm{mg} / \mathrm{dm}^{3}\right)$. The best rooting of $C$. pilosula shoots was obtained at $1 \mathrm{mg} / \mathrm{dm}^{3}$ of IBA (43\% rooting efficiency).

Two types of explants: shoot tips and nodal segments were definitely better material for the multiplication of C. pilosula than the other explants used. Only a few explants of leaves, cotyledons, hypocotyls, stems had the ability to regenerate the adventitious buds. It was not possible to regenerate buds on the roots originating from seedlings independently of the type of the cytokinins and their concentrations used.

\section{Reference}

[1] He J.Y., Zhu S., Goda Y., Cai S.Q., Komatsu K. (2014) J. Nat. Med. 68: 326-339.

[2] Gao S., Wang H., Zeng C., Hou J., Zhang Y. (2012) JCMRD 1: 16-22.

[3] He J.Y., Ma N., Zhu S., Komatsu K., Li Z.Y., Fu W.M. (2015) J. Nat. Med. 69: 1-21. 


\title{
Poster no. 34
}

\section{The influence of light on the development of Moluccella laevis cells and organs in in vitro cultures}

\author{
SYlWia ZiElińSKa ${ }^{1 *}$, WeroniKa KOZŁOWSKA ${ }^{1}$, ALEKSANDRA BOHATER ${ }^{2}$, \\ JOANNA KOLNIAK-OSTEK ${ }^{3}$, ADAM MATKOWSKI ${ }^{1}$ \\ ${ }^{1}$ Department of Pharmaceutical Biology, Wroclaw Medical University, Wrocław, Poland \\ ${ }^{2}$ Student Scientific Club no K 76, Department of Pharmaceutical Biology, Wroclaw Medical University, Wrocław, Poland \\ ${ }^{3}$ Vegetable and Plant Nutraceutical Technology, Wrocław University of Environmental and Life Sciences, Wrocław, Poland \\ *sylwia.zielinska@umed.wroc.pl
}

Moluccella laevis L. (Lamiaceae) is an annual plant, naturally occurring in the areas of Western and Central Asia (Irano-Turanian area), Turkey and the Caucasus. It is a popular decorative plant because of its magnificent green chalices which hide in their interior inconspicuous white two-lipped flowers. The aim of the experiment was to introduce for the first time Moluccella species to the in vitro conditions and check the influence of two types of illumination on the in vitro morphogenesis of $M$. leavis. Two spectra of light emitted by LED were tested: the white one and the photosynthetic active radiation range. Aseptically grown seedlings were the source of explants for the in vitro cultivation. Shoots containing the apical bud and the two axillary buds were placed on the proliferation media. In order to induce callus tissue, explants from cotyledons, leaves, petioles, hypocotyls and roots were used. The culture medium was MS-based medium without additives or supplemented with plant growth regulators (different concentrations of auxins: 1-Naphthaleneacetic acid - NAA, 2,4-Dichlorophenoxyacetic acid - 2,4-D and cytokinins: 6-Benzylaminopurine - BA, N-(2-Furylmethyl)-3H-purin-6-amine - KIN). Cultures were maintained at $25 \pm 2{ }^{\circ} \mathrm{C}$ under a 16/8-h (light/dark) photoperiod with two types of LED ilumination: lamps emitting white light (8000-10000 K) and lamps emitting light of the photosynthetically active radiation range (PAR): $730 \mathrm{~nm}, 660 \mathrm{~nm}, 630 \mathrm{~nm}, 610 \mathrm{~nm}$, $460 \mathrm{~nm}, 430 \mathrm{~nm}$. The photon flux density was $180 \mu \mathrm{M} \cdot \mathrm{m}^{-2} \cdot \mathrm{s}^{-1}$. After 28 days of cultivation, the morphogenetic response of the shoots obtained and the formation and growth of callus tissue were recorded. The highest number of shoots per explant was obtained for plants exposed to white light and grown on a culture medium supplemented with BA $(2 \mu \mathrm{M})$ and NAA $(0.5 \mu \mathrm{M})$ and for those exposed to PAR and grown with the presence of BA $(3 \mu \mathrm{M})$ and NAA $(0.5 \mu \mathrm{M})$. The best explants for dedifferentiation and callus development were hypocotyls and shoots. In white light conditions, on the callus that grew on a medium containing 2,4-D/NAA/BA the organogenesis processes occurred. The results showed that cells and shoots cultures grew more intensively under PAR light, while white light stimulated organogenesis. The composition of polyphenols in the extract was analyzed using High Performance Liquid Chromatography (HPLC) an ACQUITY Ultra Performance LCTM system (UPLC) with binary solvent manager (Waters Corporation, Milford, USA) and a Micromass Q-TOF mass spectrometer (Waters, Manchester, U.K.), equipped with an electrospray ionization (ESI) source operating in the negative and positive mode. For instrument control, the data acquisition and processing Mass LynxTM software (Version 4.1) was used. Phenolic acids: caffeic, chlorogenic, ferulic and rosmarinic were identified by HPLC. In addition, the analysis with UPLC confirmed the presence of cyanidin 3-O-galactoside and cyanidin 3-0-malonylglucoside. The developed in vitro culture methodology will be used for micropropagation, the establishment of experimental suspension cultures and phytochemical analysis of the obtained plant material. 


\title{
Epigenetic regulation of chalcone synthase isoforms expression in flax (Linum usitatissimum L.) plants under stress conditions
}

\author{
Agata Hnitecka*, Magdalena ŻUK \\ Department of Genetic Biochemistry, Faculty of Biotechnology, University of Wroclaw, Poland \\ *agata.hnitecka@uwr.edu.pl
}

Both biotic and abiotic stresses trigger defensive reactions in plants in order to overcome the effects of unfavorable environmental conditions, as well as to protect against patogens attacks. For this purpose, they produce secondary metabolites - flavonoids that act among others, as phytoalexins and phytoantycypines. These compounds are produced in plants on the flavonoid biosynthetic pathway, the key enzyme of which is chalcone synthase (CHS). The level of CHS activity is primarily controlled at the transcription level. Therefore, by examining the expression profiles of $C H S$ isoforms, we can determine their contribution to plants stress response. The results obtained by Real-Time PCR analysis indicate that the expression of chalcone synthase in flax changes under the influence of stress and that similarly to other species, isoforms differ in expression patterns.

DNA methylation is one of the epigenetic marks. Its patterns are maintained after DNA replication and cell division. DNA methylation is commonly considered as a modification silencing expression of controlled genes, especially if it is found in their promoter regions. The analysis of the chalcone synthase gene isoforms within the CCGG sequences found in the promoter, the intron and the coding regions indicates that individual cytosine residues show variable or invariable methylation patterns, when compared with plants cultivated in stress and control conditions. Invariable CCGG showed at least $90 \%$ demethylation. They probably do not take part in the regulation of the chalcone synthase gene. In contrast, variable cytosines change their methylation patterns depending on the culture conditions. They show a higher level of methylation when compared to invariant sequences. However, these changes cannot be directly identified with the changes in expression profiles of the analyzed $C H S$ isoforms.

In the next part of this research the total methylation of flax genome was determined. The reaction of plants to unfavorable conditions is a process involving the action of more than one gene. Basing on total level of methylation we could? predict the scale of ongoing regulatory mechanisms. As expected, the total methylation level showed a decrease after stress treatment.

The last experimental part involved expression analysis of genes related to epigenetic processes. The transcript levels of $C M T 1$ and $C M T 3$ methyltransferases, $H 3 K 9$ histone methyltransferase, DDM1 chromatin remodeling protein and $R O S$ and $D M E$ demethylases were evaluated.

Attempts to connect the DNA methylation patterns with the chalcone synthase expression suggest, that methylation in the gene's coding region may be regulatory, and that it may be necessary for the maintenance of the active chromatin state and gene transcription. Analyzing the total methylation profile we can predict to a large extent the level of chalcone synthase gene activity, but not of the genes responsible for epigenetic processes. In addition, the latter exhibited different expression patterns in each stress treatment, demonstrating high complexity of the ongoing epigenetic processes. 


\title{
Poster no. 36
}

\section{Biochar exploitation in in vitro culture of Alyssum montanum - morphogenetic and biochemical response of shoots on medium supplementation with biochar}

\author{
Alina Wiszniewska $^{1 *}$, AleKSANdra Koźmińska ${ }^{1}$, Alicja Kulińska ${ }^{2}$, Ewa Hanus-Fajerska ${ }^{1}$ \\ ${ }^{1}$ Unit of Botany and Plant Physiology, Institute of Plant Biology and Biotechnology, \\ Faculty of Biotechnology and Horticulture, University of Agriculture in Kraków, Poland \\ ${ }^{2}$ Biotechnology Student, Faculty of Biotechnology and Horticulture, University of Agriculture in Kraków, Poland \\ *a.wiszniewska@urk.edu.pl
}

Biochar is a carbon-rich product of biomass pyrolysis. Its application as a soil amendment enhances plant productivity, especially under abiotic stresses such as drought and soil contamination. Due to its physico-chemical properties, biochar could also be exploited as a cost-effective medium supplement for in vitro culture, substituting activated charcoal. Therefore the aim of this study was to evaluate the morphogenetic and biochemical effects of medium supplementation with two types of activated biochar in a shoot culture of Alyssum montanum calamine ecotype, in the absence and presence of lead nitrate.

Shoots were cultured in a modified WPM medium, with or without of $1.0 \mathrm{mM} \mathrm{PbNO}_{3}$, supplemented with either $0.6 \mathrm{~g} / \mathrm{dm}^{3}$ activated charcoal (AC) (Sigma Aldrich) or $0.6 \mathrm{~g} / \mathrm{dm}^{3}$ activated biochar (AB). Biochars were produced in the International Institute for Sustainability in Rio de Janeiro (IIS), Brazil. Biochar activation was obtained using water (water-activated biochar - W-AB) or cattle urine (urine-activated biochar - U-AB). After 6 weeks of culture, the following growth and biochemical parameters were assessed: the micropropagation coefficient (MC), biomass accretion, the content of phenolic compounds, photosynthetic pigments and soluble sugars, and the level of lipid peroxidation and radical scavenging activity.

The application of activated biochars enhanced the proliferation efficiency and biomass accretion in $A$. montanum plants cultured without $\mathrm{Pb}$. The MC increased from 1.2 in control medium to 7.1 and 5.1 in media supplemented with $\mathrm{U}-\mathrm{AB}$ and $\mathrm{W}-\mathrm{AB}$, respectively. In cultures without $\mathrm{Pb}$, the $\mathrm{MC}$ and biomass accretion were not affected by biochar application. The biochemical response differed for plants cultured in the presence or absence of $\mathrm{Pb}$. The content of phenolic compounds, soluble sugars and photosynthetic pigments decreased in shoots from $\mathrm{Pb}(-)$ media supplemented with biochars. Also, a radical scavenging activity rapidly declined in the presence of biochars. In contrast, in $\mathrm{Pb}(+)$ media supplemented with biochars the content of phenolic compounds significantly increased, together with a radical scavenging activity. Soluble sugars declined in shoots from W-AB medium, but greatly increased in the presence of U-AB.

Our results indicate that biochar can be used as medium supplement in in vitro culture. However, we observed that various biochars may exert different effects on cultured shoots. An important factor responsible for this is the method of biochar production and activation, which significantly influences biochar properties and has a further effect on cultured plants. In $A$. montanum cultures, biochars stimulated a biochemical response in stressful conditions, and accelerated the shoot growth in the absence of a toxic substance in the medium.

\section{Acknowledgements}

The research was financed by the Polish Ministry of Science and Higher Education. Authors thank Dr. Agnieszka E. Latawiec from the International Institute for Sustainability in Rio de Janeiro (IIS), Brazil for providing biochar samples. 
Poster no. 37

\title{
Structural and metabolic adaptation of contrasting Silene vulgaris ecotypes to heavy metal stress
}

\author{
Ewa Muszyńska ${ }^{1 *}$, MirosŁawa Górecka ${ }^{1}$, Mateusz Labudda ${ }^{2}$, \\ EWA HANUS-FAJERSKa ${ }^{3}$, ANNA KOSZELNIK-LESZEK ${ }^{4}$ \\ ${ }^{1}$ Department of Botany, Faculty of Agriculture and Biology, Warsaw University of Life Sciences - SGGW, Poland \\ ${ }^{2}$ Department of Biochemistry, Faculty of Agriculture and Biology, Warsaw University of Life Sciences - SGGW, Poland \\ ${ }^{3}$ Institute of Plant Biology and Biotechnology, Faculty of Biotechnology and Horticulture, University of Agriculture in Krakow, Poland \\ ${ }^{4}$ Department of Botany and Plant Ecology, Wrocław University of Environmental and Life Sciences, Poland \\ * ewa_muszynska@sggw.pl
}

Three Silene vulgaris ecotypes representing calamine, serpentine and non-metallicolous populations were evaluated for their response to various media compositions applied for shoot multiplication and callus induction. In the first experimental step we elaborated culture conditions identical for all tested ecotypes in order to standardize in vitro methods for further research. It was found that the most balanced development of shoots in all studied ecotypes was obtained on MS medium containing $0.1 \mathrm{mg} / \mathrm{dm}^{3} \mathrm{NAA}$ and $0.25 \mathrm{mg} / \mathrm{dm}^{3}$ BAP. In turns, the most abundant callus formation was observed on MS medium enriched with $0.5 \mathrm{mg} / \mathrm{dm}^{3} \mathrm{NAA}$ and $5.0 \mathrm{mg} / \mathrm{dm}^{3}$ BAP (when abaxial leaf surface was put on the medium). In the next experimental step, we applied an in vitro culture as a convenient laboratory tool to reveal $S$. vulgaris strategies involved in counteraction metal toxicity at the cellular and physiological level. Therefore, shoot cultures were treated with $30.0 \mu \mathrm{M} \mathrm{Pb}\left(\mathrm{NO}_{3}\right)_{2}$ or $1.0 \mathrm{mM} \mathrm{NiSO}_{4}$. The metal concentrations corresponded to the amounts of soluble forms of a respective element ascertained in natural conditions. As a control, a propagation medium without heavy metal salts was used. After four weeks of treatment, the anatomical and ultrastructural analyses under a light, confocal and electron microscope were performed for the localization of phenolic compounds, flavan-3ols and lignins in cultivated shoots. Moreover, the localization of lead and nickel ions was examined according to a histochemical method with dithizone and dimethyloglioxyme, respectively. The studies were complemented by spectrophotometric measurements of the phenolic compounds concentration and the photosynthetic pigments contents. The application of salt containing $\mathrm{Ni}$ ions resulted in a strong growth inhibition and led to the culture death independently of the tested ecotype. On the contrary, the shoots development in the presence of $\mathrm{Pb}$ ions proved to be macroscopically undisturbed, even though the micropropagation efficiency decreased in the following order: non-metallicolous $(\mathrm{MC}=1.6)<$ serpentine $(\mathrm{MC}=2)<$ calamine $(\mathrm{MC}=3)$ ecotype. Despite this, the electron microscope analysis revealed some ultrastructural alterations under $\mathrm{Pb}$ stress. The most pronounced changes noticed in leaves of non-metallicolous ecotype were manifested by a shrunken tonoplast, areas of lower electron density inside mitochondria or a looser arrangement of chloroplasts with huge plastoglobuli. While in leaves sampled from calamine and serpentine lines treated with $\mathrm{Pb}$ ions disturbances in the cell ultrastructure were observed only sporadically. The most significant discrepancy between these two metallicolous ecotypes was connected with the chloroplast ultrastructure occurrence of membrane invaginations and dark deposits on the tonoplast, probably phenols in nature, that appeared frequently in calamine specimens. These observations corresponded to the quantitative analysis of the chlorophyll content and the total phenolic compound concentration in leaves. Moreover, in the course of a histochemical detection of $\mathrm{Pb}$ ions, red color complexes of metal-dithizonate were noticed in the root stela of non-metallicolous specimens, while in calamine and serpentine ones it was the endodermis cells that were stained the most. The results obtained under in vitro model indicated the diversity of non-metallicolous and metallicolous populations at the anatomical and ultrastructural level, which was reflected in different responses of the examined ecotypes to $\mathrm{Pb}$ ions.

\section{Acknowledgement}

The research was financed by the Polish National Science Centre within Project No. 2017/01/X/NZ8/00382. 


\title{
Poster no. 38
}

\section{Effect of LED light pretreatment of sugar beet donor plants on haploid embryos induction}

\author{
KAMILA KOZAK-STANKIEWICZ ${ }^{1 *}$, KaTARZYNa WróBleWSKA ${ }^{1}$, JOANNA NOCEN ${ }^{2}$, \\ STANISŁAW KARPIŃSKI ${ }^{3}$, MAGDALENA SZECHYŃSKA-HEBDA ${ }^{4}$, ADAM SITARSKI ${ }^{1}$ \\ ${ }^{1}$ Kutno Sugar Beet Breeding Company, Kłodawa, Poland \\ ${ }^{2}$ Plant Breeding and Acclimatization Institute - National Research Institute \\ National Centre for Plant Genetic Resources, Radzików, Poland \\ ${ }^{3}$ Department of Plant Genetics, Breeding and Biotechnology, Faculty of Horticulture, Biotechnology and Landscape Architecture, \\ Warsaw University of Life Sciences - SGGW, Poland \\ ${ }^{4}$ The Franciszek Górski Institute of Plant Physiology, Polish Academy of Sciences, Kraków, Poland
}

Since the early 80 's a significant progress has been made to produce homozygous lines owing to the use of in vitro unpollinated ovules. It is well known that the gynogenesis rate is affected by various environmental and genetic factors. As the reports published so far show only a limited number of aspects related to the growth condition of the donor plant have been examined. The basis for the environmental effect on the sugar beet donor plant responsiveness to in vitro haploid production like eg. the source of light has not been determined. It may be mediated by alterations in the developmental pattern of the ovule because haploid embryo induction is sensitive to the stage of development of ovules at the time of isolation. Rapid staging methods based on the bud size, the days before anthesis or the days of the chilling treatment to collect suitable inflorescences are only partially correlated with the developing embryo yield. The aim of this study was to find out whether the fitness of maternal plants could be affected by the source of light in the context of increasing gynogenic potential. Donor plants of 12 sugar beet breeding lines were grown in a climate chamber. Uniform condition were set for all tested genotypes to eliminate the influence of environmental differentiation except the light sources. Two different artificial light treatments were applied: standard high-pressure sodium lamps (HPS) of $400 \mathrm{~W}(6000 \mathrm{~K}, 55,000 \mathrm{~lm})$ and a new prototype LED lighting apparatus according to the patent application WO 2011154522 A1 [Karpinski and Szechynska-Hebda, 2011]. The obtained results indicated that the light-emitting diode applied in this experiment during the donor plant growth was able to increase the efficiency of haploid induction. A proportion of the ovules from the buds grown under the LED's followed a different pathway of development than those from plants grown under HPS condition, which affected their physical properties and their ability to develop into embryos in in vitro culture. The results of this study support the concept that at least a portion of the responsiveness of unpollinated ovules could be determined by physical treatment before in vitro culture.

The work was supported by PBS3/A9/37/2015 project operating within the Applied Research Programme of the National Centre for Research and Development. 
Poster no. 39

\title{
Morphogenetic response of Gerbera jamesonii Bolus. to different light intensities in vitro
}

\author{
Monika Cioć *, Andrzej Kalisz ${ }^{2}$, Bożena Szewczyk-TaraneK ${ }^{1}$, Bożena PaWlowska ${ }^{1}$ \\ ${ }^{1}$ Department of Ornamental Plants, University of Agriculture in Krakow, Poland \\ ${ }^{2}$ Department of Vegetable and Medicinal Plants, University of Agriculture in Krakow, Poland \\ *m.cioc@ogr.ur.krakow.pl; andrzej.kalisz@urk.edu.pl
}

Light in in vitro cultures affects photomorphogenesis, directing plant growth and development, as plant photoreceptors are sensitive to light quality and wavelength. The mixed spectrum of red and blue light is the most important during micropropagation [4]. Although the morphogenetic response of plants occurs at low light intensities, significantly lower than those required for photosynthesis, light intensity is also very important during in vitro cultures [1].

The object of this study was to evaluate the influence of light intensities on Gerbera jamesonii "Big Apple" shoots multiplicated in vitro. Red (70\%) and blue (30\%) light mixtures emitted by light emitting diodes (LEDs) were tested in different photosynthetic photon flux densities (PPFD): 40, 80 and $120 \mu \mathrm{mol} / \mathrm{m}^{2} \mathrm{~s}$. White fluorescent light was used as a control $\left(40 \mu \mathrm{mol} / \mathrm{m}^{2} \mathrm{~s}\right)$. The culture was conducted on Murashige and Skoog [3] medium supplemented with 3\% sucrose, 5\% Bioagar, $0.5 \mu \mathrm{M}$ NAA and $2.5 \mu \mathrm{M}$ BA. After six weeks, biometrical observations of proliferated shoots and leaf morphometric parameters (WinDIAS leaf area meter) were performed. Photosynthetic pigment contents were also analyzed (spectrophotometric method [2]).

The results showed that the tested light intensities affected the parameters studied. The regeneration rate did not differ under control and LED light conditions ( 40 and $80 \mu \mathrm{mol} / \mathrm{m}^{2} \mathrm{~s}$ ) and amounted to 6.2-6.8. However, the highest PPFD applied ( $\left.120 \mu \mathrm{mol} / \mathrm{m}^{2} \mathrm{~s}\right)$ inhibited shoot multiplication (4.2) and their height. The number of developed leaves was the same in each tested combination.

The use of $40 \mu \mathrm{mol} / \mathrm{m}^{2} \mathrm{~s}$ LED light resulted in a higher level of all photosynthetic pigments compared to the same intensity of fluorescent control light. However, three-fold increase in PPFD $\left(120 \mu \mathrm{mol} / \mathrm{m}^{2} \mathrm{~s}\right)$ resulted in more than two-fold reduction in the pigment content.

The tested light combinations had an effect on the morphometric parameters of leaves. Enhancing light intensity resulted in an increased leaf blade area and width. Circular and elongation factors were also the highest at $120 \mu \mathrm{mol} / \mathrm{m}^{2}$ s PPFD. For these parameters, the control fluorescent light produced the worst results. The opposite tendency was observed for petiole length.

\section{References}

[1] Alvarenga I.C.A., Pacheco F.V., Silva S.T., Bertolucci S.K.V., Pinto J.E.B.P. (2015) Plant. Cell. Tiss. Org. 122: 299-308.

[2] Lichtenthaler H.K., Buschmann C. (2001) [In:] Current protocols in food analytical chemistry. Ed. Lichtenthaler H.K., New York: Wiley: 171-178.

[3] Murashige T., Skoog F. (1962) Physiol. Plant. 15: 473-497.

[4] Pawłowska B., Żupnik M., Szewczyk-Taranek B., Cioć M. (2018) Hort. Environ. Biotechnol. 59: 115-123.

This study was supported by the Polish Ministry of Science and Higher Education: DS 3500/KRO/2017. 


\title{
Poster no. 40
}

\section{Multiplication of shoots and callus induction of Stevia rebaudiana Bertoni on media with an addition of active charcoal}

\author{
MAGDALENA DYDUCh-SIEMIŃSKA *, JACEK GAWROŃSKI \\ Institut of Plant Genetics Breeding and Biotechnology, University of Life Sciences in Lublin, Lublin, Poland \\ *magdadyduch@wp.pl
}

Stevia rebaudiana Bertoni is a perennial herb and belongs to the family Asteraceae. Stevia leaves are a source of basic chemical components and valuable biologically active substances, and above all, steviol glycosides which give the plant a sweet taste. The main compounds with a sweet taste found in the leaves are stevioside, rebaudioside $\mathrm{A}$ and dulcoside. The growing market demand for plant metabolites and the limitations of conventional methods of obtaining them have triggered attempts to find alternative, more efficient ways of their production. Such a chance is created by the in vitro cultivation of plant cells and tissues. Currently, much attention is devoted to research on the optimization of in vitro culture conditions, striving to obtain a large number of plants or biomass from which plant metabolites can be obtained with greater efficiency. Activated charcoal (AC) has a very fine network of pores with a large inner surface area on which many substances can be adsorbed. AC is often used in tissue culture to improve cell growth and development. The effect of $\mathrm{AC}$ on the growth regulator uptake is still unclear but it is believed that AC may gradually release certain adsorbed products, such as nutrients and growth regulators which become available to plants. The aim of this study was to investigate the effect of the addition of $\mathrm{AC}$ on the media used for micropropagation and callus induction of Stevia rebaudiana Bert. in in vitro cultures. S. rebaudiana sterile seedlings derived from in vitro cultures were used to initiate the culture. Shoots and leaves obtained from them were the source of explants for micropropagation and callus induction. Explants were inoculated in MS (Murashige and Skoog) media supplemented with growth regulators. For the micropropagation MS medium $+0.5 \mathrm{mg} / \mathrm{dm}^{3}$ BAP $+0.25 \mathrm{mg} / \mathrm{dm}^{3}$ kinetin (control) and medium of the same composition with AC $\left(3.0 \mathrm{~g} / \mathrm{dm}^{3}\right)$ were used. For callus induction MS medium $+2.0 \mathrm{mg} / \mathrm{dm}^{3} \mathrm{NAA}+\mathrm{mg} / \mathrm{dm}^{3} \mathrm{BAP}$ (control) and medium of the same composition with AC $\left(3.0 \mathrm{mg} / \mathrm{dm}^{3}\right)$ were used. In micropropagation on the control medium regeneration and shoot multiplication from the initial explant was observed. At the base of the regenerated shoots callus tissue was formed. On the AC medium shoot regeneration without multiplication was observed. The shoots were thicker and had large well-developed leaf blades and a root system. In the case of callus induction on the medium with the addition of AC, callus appeared only at the place of cutting in a very small amount. On the control medium, callus was formed on the entire surface of primary explants. The resulting callus was green-white and had a hard, globular structure. In the micropropagation of stevia as well as in the experiment with callus induction, AC inhibited both the production of shoots and the callus formation. The obtained results indicate that in this experiment the growth regulators used in the tested media were adsorbed by $\mathrm{AC}$ and become unavailable for explants. 
Poster no. 41

\title{
The influence of activated charcoal and ascorbic acid on the growth and morphological features of Paphiopedilum sp. orchids in vitro
}

\author{
Monika PoniewoziK *, Marzena Parzymies, Pawee Szot \\ Department of Ornamental Plants, Dendrology and Landscape Architecture, University of Life Sciences in Lublin, Poland \\ *monika87_1987@o2.pl
}

Paphiopedilum Pfitzer belongs to the Orchidaceae family. It is a very interesting orchid used as a decorative pot plant and in floristic compositions. Orchids from the Paphiopedilum genus have original and interesting flowers with lips similar to a shoe and irregular spots on flowers. Some of the varieties characterize ornamental leaves as well.

Paphiopedilum is commonly propagated through in vitro techniques. However, the quality of the plants might be a serious problem during cultivation. Due to browning of media around the basal part of plant, it might be assumed that phenolic compounds were present, therefore the research on the use of anti-oxidative substances was undertaken.

The aim of the study was to estimate the possibility of using activated charcoal (AC) and ascorbic acid (AA) in the tissue culture of Paphiopedilum sp. The experiment was to evaluate the effect of these compounds added to a medium on the growth and morphological features of slipper orchids microplants.

The starting material for the initiation of the tissue culture were microplants derived from a stabilized culture, obtained by the asymbiotic method of seed germination in vitro. They were $5 \mathrm{~mm}$ long, with 1-2 leaves. The explants were placed inside Erlenmeyer flasks on 1/2 MS (Murashige and Skoog, 1962) media with an addition of kinetin (KIN) in a concentration of $5.0 \mathrm{mg} / \mathrm{dm}^{3}$ and $1.0 \mathrm{mg} / \mathrm{dm}^{3}$ of benzyladenine (BA) and supplemented with AA in concentrations of: 10,20 and $30 \mathrm{mg} / \mathrm{dm}^{3}$ or AC: $1.0,2.0$ or $4.0 \mathrm{~g} / \mathrm{dm}^{3}$. The medium without the anti-oxidants was treated as the control. The $\mathrm{pH}$ of the media was adjusted to 5.8 . The medium was solidified with $6.75 \mathrm{~g} / \mathrm{dm}^{3}$ of agar. The flasks were placed in a growing room at the temperature of $28 \pm 2{ }^{\circ} \mathrm{C}, 16$-hour photoperiod and light intensity of $30 \mu \mathrm{mol} / \mathrm{m}^{2} \mathrm{~s}$.

The most multiplied plants were when medium was supplemented with $2 \mathrm{~g} / \mathrm{dm}^{3}$ of activated charcoal, however the obtained plants were characterized by the smallest leaves $(7.33 \mathrm{~mm}$ long and 3.04 wide). The addition of 20 , $30 \mathrm{mg} / \mathrm{dm}^{3}$ of AA and CM (control medium) promoted formation of the biggest number of leaves (3.23; 3.75 and 3.76 , respectively). The media supplemented with $30 \mathrm{mg} / \mathrm{dm}^{3}$ of AA or $1.0 \mathrm{~g} / \mathrm{dm}^{3}$ of AC influenced the elongation of leaves $(12.41 ; 12.46 \mathrm{~mm}$ respectively). The widest leaves were observed on the control medium and with addition of $1.0 \mathrm{~g} / \mathrm{dm}^{3}$ of $\mathrm{AC}$ (5.03 mm and $4.82 \mathrm{~mm}$ respectively). Plants cultivated in presence of activated charcoal, no matter their concentration, were characterized by the highest number of roots and fresh weight of the above and underground of microplantlets. It was also noted that most plants produced roots in the presence of 2.0 and $4.0 \mathrm{~g} / \mathrm{dm}^{3}$ of AC (95 and 95\% respectively). The longest roots were observed on the medium supplemented with $1 \mathrm{~g} \cdot \mathrm{dm}^{-3} \mathrm{AC}(10.86 \mathrm{~mm})$. Significantly shorter roots were noted when plants were cultivated on the control media or in the presence of ascorbic acid.

Taking into consideration the multiplication rate of Paphiopedilum sp. plants in the tissue culture and their quality, it might be recommended to add $1.0-2.0 \mathrm{~g} / \mathrm{dm}^{3}$ of activated charcoal to the media. 


\title{
Poster no. 42
}

\section{Different acclimatization strategies of two genus from the family Droseraceae to high light intensity cultivated in vitro}

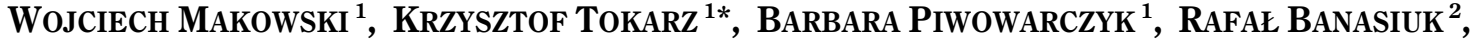 \\ ALEKSANDra KRólicka ${ }^{2}$, Michą DZiURKa ${ }^{3}$, RENATa WOJCIECHOWSKa ${ }^{1}$ \\ ${ }^{1}$ Institute of Plant Biology and Biotechnology, Faculty of Biotechnology and Horticulture, University of Agriculture in Krakow, Poland \\ ${ }^{2}$ Department of Biotechnology, Intercollegiate Faculty of Biotechnology, University of Gdansk and Medical University of Gdansk, Poland \\ ${ }^{3}$ The Franciszek Górski Institute of Plant Physiology, Polish Academy of Science, Kraków, Poland \\ *km.tokarz.ipbb@gmail.com; rafal.banasiuk@biotech.ug.edu.pl; m.dziurka@ifr-pan.edu.pl
}

Dionaea muscipula J. Ellis (Venus flytrap) and Drosera peltata Smith (shield sundew) are carnivorous plants belonging to the family Droseraceae, commonly known as unique ornamental plants and herbs used in folk medicine from centuries. Medical properties of these plants result from their ability to produce derivatives of phenolic compounds, among others plumbagin (1,4-naphtoquinone).

In natural conditions Venus flytrap and shield sundew occur on moistly, nutrient poor and open sun habitats where they are exposed to high light intensity. Plant reactions to excess light include anatomical and morphological changes and increased synthesis of secondary metabolites. The acclimatization mechanisms of plants from the family Droseraceae to high light intensity have not been described yet, therefore the aim of presented study was to characterize their strategies for dealing with excess light intensity in the context of metabolism of secondary metabolites.

Dionaea muscipula and D. peltata plants cultivated in in vitro conditions on $1 / 2$ MS medium, with $3 \%$ of sucrose ( $\mathrm{pH}=5.5$ adjusted prior autoclaving) without growth regulators, under fluorescence light of $50 \mu \mathrm{mol} \cdot \mathrm{m}^{-2} \cdot \mathrm{s}^{-1}$ photosynthetic photon flux density (PPFD) were used in experiment. Plants were transferred into fresh medium and cultivated under $50 \mu \mathrm{mol} \cdot \mathrm{m}^{-2} \cdot \mathrm{s}^{-1} \mathrm{PPFD}$ (control) and higher light - exposed to $120 \mu \mathrm{mol} \cdot \mathrm{m}^{-2} \cdot \mathrm{s}^{-1} \mathrm{PPFD}$ (high light intensity). After 30 days of cultures, the dry matter in both examined plants were estimated. Chlorophyll $a$ and $b$, carotenoids, total content of phenolic compounds, proline and malondialdehyde (MDA) were examined using spectrophotometric methods, quantity of plumbagin was measured with high performance liquid chromatography, while jasmonic acid (JA) and salicylic acid (SA) were measured using ultra-high performance liquid chromatography tandem mass spectrometry.

Application of the higher light intensity caused a statistically significant increase of chlorophyll $a, b$ and carotenoids contents in both examined genus. Drosera peltata accumulated significantly more dry matter, proline, plumbagin and salicylic acid, simultaneously showing significantly higher levels of lipid peroxidation than control plants, whereas in D. mucipula tissues the total content of phenolic compounds and plumbagin significantly increased.

Presented results might indicate two different acclimatization strategies of the examined plants to increased light intensity: in D. muscipula phenolic compounds act as a screening metabolites and/or antioxidant agents. In the case of $D$. peltata, the physiological reaction is associated with increased synthesis of salicylic acid (that as signaling compound induces proline synthesis) and increased activity of reactive oxygen species leads to lipid peroxidation and malondialdehyde accumulation. On the other hand, presented results show that high intensity of radiation can be used as an abiotic elicitor of phenolic compounds in tissue culture of selected plants from the family Droseraceae. 
Poster no. 43

\title{
Effect of sucrose treatment on somatic embryogenesis in Cyathea delgadii Sternb.
}

\author{
Wojciech Tomaszewicz * MaŁgorzata Grzyb, Jan JarosŁaw Rybczyński, AnNa MikUŁa \\ Department of Plant Experimental Biology, \\ Polish Academy of Sciences Botanical Garden - Center for Biological Diversity Conservation in Powsin, Poland \\ *w.tomaszewicz@obpan.pl
}

Somatic embryogenesis (SE) is the developmental pathway by which plant somatic cells differentiate into somatic embryos. Cyathea delgadii is the first fern for which SE has been described. Up till now, it was known, that somatic embryos of $C$. delgadii are initiated from the single epidermal cells. Our latest research revealed that they can also be formed by multicellular pathway [1]. However, in this way of somatic embryo production, the efficiency of SE is very low. Thus, the present study was undertaken to investigate the effect of sucrose treatment to improve SE induced on the internode explants of $C$. delgadii. Osmotic stress shows beneficial effect on the SE efficiency in many spermatophytes. In some cases, this factor can effectively replace the plant growth regulators that are conventionally using to stimulate the morphogenetic capacity in vitro [2].

The source of plant material were internodes excised from the first frond of five-month-old etiolated sporophytes cultured on $1 / 2$ MS medium supplemented with $2 \%(\mathrm{w} / \mathrm{v})$ sucrose, without any plant growth regulators. Internodes were stressed directly after their excision or after 2-day-long culture on the 1/2 MS with $1 \%$ sucrose. The effect of sucrose on SE was examined by treating the internode explants with the aqueous solutions of $0.3,0.4,0.5,0.6$ or $0.7 \mathrm{M}$ sucrose for $15,30,45$ or $60 \mathrm{~min}$. Following stress treatment, the explants were cultured on the medium mentioned above, in constant darkness, at $24^{\circ} \mathrm{C}$. In order to evaluate the embryogenic potential of internode explants, 60 explants for each treatment were examined. The efficiency of SE was expressed as the percentage of responding explants, the number of somatic embryos per responding explant (after 1 month of culture), and as the somatic embryo production capacity index (SEPCI; calculated by multiplying the percentage of responding explants by the number of somatic embryos formed per explant and then dividing the result by 100). For visualization and identification of somatic embryo origin and their development, the light and environmental scanning electron microscopes were used.

The SEPCIs of control explants were equal to 2.6-2.7. When internode explants were treated with $0.4 \mathrm{M}$ sucrose directly after their excision, the highest SEPCIs were about 4 -fold higher for $45 \mathrm{~min}$. and $60 \mathrm{~min}$. treatment, in comparison to the control. The frequency of explants producing somatic embryos decreased with increasing the sucrose concentration and prolonging the treatment time. Internodes treated with sucrose after two days of incubation on 1/2 MS medium were characterized by high frequency of somatic embryogenesis. The SEPCI of explants treated with $0.4 \mathrm{M}$ sucrose for $45 \mathrm{~min}$. was equal to 32.0. Microscopic analysis revealed that somatic embryos originated from many cells of both the epidermis and cortex. Sucrose treatment significantly increased the SE efficiency by changing the multicellular pathway of embryo origin to the unicellular pathway. To the best of our knowledge, this is the first report about this occurrence.

This research provides an excellent model for the study, which can help broaden our knowledge of somatic embryogenesis. This method for the in vitro reproduction of tree ferns may be valuable for the rapid propagation of these plants both for conservation and commercial purposes.

\section{References}

[1] Mikuła A., Grzyb M., Tomiczak K., Rybczyński J.J. (2018) [In:] Current Advances in Fern Research. Ed. Fernández H. Gewerbestrasse, Springer: 121-137.

[2] You X.L., Yi J.S., Choi Y.E. (2006) Protoplasma 227: 105-112. 


\title{
Poster no. 44
}

\section{Sodium nitroprusside affects root organogenesis in fiber flax (Linum usitatissimum L.) cultures in vitro}

\author{
Aneta AdamczuK *, Irena Siegień, Iwona Ciereszko \\ Department of Plant Physiology, University of Białystok, Poland \\ *a.adamczuk@uwb.edu.pl
}

Plant in vitro culture is a convenient material for investigating the mechanisms of nitrogen oxide (NO) in the regulation of plant growth and development. However, there are only few studies about effects of NO on plant regeneration in in vitro cultures. Positive effect on shoots and roots organogenesis were obtained e.g. in the cultures of flax [1] or vanilla [2]. These experiments refer to improvement of organogenesis after exposure to NO donors, including sodium nitroprusside (SNP) and S-nitrozo-N-acetyl-DL-penicillamine (SNAP).

The aim of our study was to determine the effect of SNP on the regeneration efficiency in fiber flax in vitro cultures and the answer to the following questions: 1 ) whether the vapours from the SNP (containing NO) generates oxidative stress in tissues; 2) to what extent the observed changes in root organogenesis depend on the activity of the antioxidant system?

In cultures of fiber flax (Linum usitatissimum L., cv. Selena), the differences in rhizogenesis were observed due to the action of vapors from $5 \mathrm{mM}$ SNP. Hypocotyl explants were cultured on Gamborg (B5) medium with PGRs: $\alpha$-naphthylacetic acid (NAA, $\left.1 \mathrm{mg} \cdot 1^{-1}\right)$ and 6-benzyladenine $\left(0.025 \mathrm{mg} \cdot 1^{-1}\right)$ or medium without PGRs, and then exposed to the SNP. The antioxidant capacity of tissue to the reduction of stable 1,1-diphenyl-2-picrylhydrazine free radical (DPPH), TBARS, and $\mathrm{H}_{2} \mathrm{O}_{2}$ content, and antioxidant enzymes activity: catalase (CAT), superoxide dismutase (SOD) and its isoforms were determined.

The vapours from the SNP solution were involved to improvement of tissue regeneration, both in NAA-enriched root cultures and in cultures grown without PGRs. Experiments with c-PTIO have confirmed NO's contribution to regulation of organogenesis in fiber flax. Cultures treated with $\mathrm{NO}$ showed better antioxidant capacity than control in reaction with DPPH. At the early stage of organogenesis these cultures were also characterized by reduced $\mathrm{H}_{2} \mathrm{O}_{2}$ and TBARS content. Increased synthesis of $\mathrm{H}_{2} \mathrm{O}_{2}$ was observed at the later stage of growth in PGRs-free cultures. SNP fumigation did not affect SOD and CAT activity in most cases, but in the later stage of culture growth, the increased activity of MnSOD was observed. The stimulation of rhizogenesis efficiency, as the result of NO action, probably occurred due to interaction of the NO and PGRs in medium, or via synthesis of hormones (e.g. cytokinins) and/or signalling molecules (e.g. $\mathrm{H}_{2} \mathrm{O}_{2}$ ) stimulation.

\section{References}

[1] Kalra C., Babbar S.B. (2010) Plant Cell Tiss. Organ Cult. 103: 353-359.

[2] Tan B.C., Chin C.F., Alderson P. (2013) In Vitro Cell. Dev. Biol. Plant 49: 626-630. 
Poster no. 45

\title{
Expression profile of the $H V S N A C 1$ gene (Stress-responsive $N A C$ ) in barley during cold stress treatment
}

\author{
Paulina KościelniaK *, Marzena Kurowska, Iwona SzareJKo \\ Department of Genetics, Faculty of Biology and Environmental Protection, University of Silesia in Katowice, Poland \\ *pkoscielniak1@us.edu.pl
}

Plant growth during cold stress induces the expression of numerous transcription factors (TF), which up-regulate or down-regulate a series of downstream genes. This cellular transcriptional network controls and modulates stress adaptive pathways. One of the major plant transcription factor families is NAC. Its name comes from the names of the first identified members of the NAM family (no apical meristem), ATAF1-2 (Arabidopsis thaliana transcription factor) and CUC2 (cup-shaped cotyledon). Proteins belonging to this family contain a highly conserved N-terminal dimerization and DNA-binding domain called the NAC domain. The $H_{V} S N A C 1$ gene analyzed in the study is a member of the NAC transcription factor family. Cold stress is a major environmental factor that affects plant growth, development and productivity. It adversely affects the growth and development of plants, directly, through the inhibition of metabolic reactions and indirectly, through the induction of oxidative stress. Barley (Hordeum vulgare) occupies the fourth place in terms of cultivated area in Poland and in the world, which shows that it is an important cereal crop. Understanding the molecular basis of resistance to cold stress in cereals is a very important aspect of research aimed at obtaining varieties with increased tolerance to cold stress. The aims of the study was to: a) investigate whether the expression of the $H_{V S N A C 1}$ gene in barley during cold stress is induced in different tissues (of leaf and root), and b) define the expression profile of this gene during different time points of stress treatment.

"Sebastian" cultivar of barley was used in the study. The 6-day-old barley seedlings grown in an aeroponics in a growth chamber at $21^{\circ} \mathrm{C} / 18^{\circ} \mathrm{C}, 16 / 8 \mathrm{~h}$ photoperiod were treated with cold stress by sudden cooling in ice and then transferring them into a growth chamber at $4^{\circ} \mathrm{C}$. The leaf and root tissues of seedlings for gene expression analyzes were collected at the different time points of stress treatment: 1, 6 and 24 hours and a control conditions. The total RNA from each barley sample was extracted using TriPure Isolation Reagent and next used to reverse transcription and qPCR reaction. The value of the relative expression level was normalized to a reference gene $A D P$ ( $A D P$ ribozylation factor 1 ). The LinReq software was used for data analysis. The relative expression level was calculated using the expression of a reference gene and the gene of interest under control conditions, according to the deltadelta $\mathrm{Ct}$ method. Three biological replications were used for the gene expression analysis, each sample was analyzed in two technical replicates.

We have shown that the relative level of $H_{V S N A C 1}$ gene expression increased during cold stress treatment in all investigated time points, both in leaf and root tissues. Interestingly, the expression pattern of the analyzed gene is very similar in both tissues to the highest up-regulation peak at 6 hours of cold treatment, 9 and 5 times in leaf and root, respectively. The $H_{V S N A C 1}$ is a transcription factor involved in the cold stress response in barley. 


\title{
Influence of different concentrations of sucrose on the growth and production of iridoid and phenylethanoid glycosides in hairy root cultures of Rehmannia elata N.E. Brown ex Prain.
}

\author{
Magdalena PiniaK \\ Department of Biology and Pharmaceutical Botany, Medical University of Łódź, Poland \\ magdalena.piniak@stud.umed.lodz.pl
}

Rehmannia elata N.E. Brown ex Prein. and other species from the genus Rehmannia (Orobanchaceae family) are perennial plants naturally occurring on mountain slopes and trails in China, Korea and Japan. The raw material obtained from the roots of some members of Rehmannia genus, called Rehmanniae Radix (also known as Di Huang), is officially listed in the Chinese Pharmacopoeia [3]. Rehmanniae Radix is widely used in Chinese traditional medicine as anti-anemic, anti-pyretic, anti-inflammatory, hypoglycemic, anti-hypertensive and anti-senescence agents [4]. The biological activity and therapeutical effects are related mainly to the presence of iridoid, phenylethanoid glycosides as well as polysaccharides (rehmannosides A-D) [2, 4]. The aim of the present study was to investigate the influence of different sucrose concentrations $\left(20,30,40\right.$ or $50 \mathrm{~g} / \mathrm{dm}^{3}$ ) on biomass (fresh and dry mass in $\mathrm{g} /$ flask) as well as on the production of iridoid and phenylethanoid glycosides in hairy roots of $R$. elata after 28 days of growth in liquid Woody Plant Medium (WPM) [1] without growth regulators. Additionally, growth indexes for fresh and dry weight were calculated for the roots after 28 days of growth. It was observed that the highest average fresh weight of the roots $(6.43 \pm 0.88 \mathrm{~g} /$ flask $)$ was in a medium with $20 \mathrm{~g} / \mathrm{dm}^{3}$ of sucrose. Slightly lower fresh weights (6.01-6.22 g/flask), but without statistical differences $(P \leq 0.05)$, were achieved in media with higher (30 and $40 \mathrm{~g} / \mathrm{dm}^{3}$ ) sucrose concentrations. A statistically lower average fresh weight was determined in a medium supplemented with $50 \mathrm{~g} / \mathrm{dm}^{3}$ of sucrose ( $5.36 \pm 0.90 \mathrm{~g} /$ flask). Dry weight of the hairy roots increased with the increase of sucrose concentration on the medium and ranged between $0.62 \mathrm{~g} / \mathrm{flask}\left(20 \mathrm{~g} / \mathrm{dm}^{3}\right.$ of sucrose $)$ and approx. $0.80 \mathrm{~g} / \mathrm{flask}$ (40 and $50 \mathrm{~g} / \mathrm{dm}^{3}$ of sucrose). Growth indexes varied depending on the sucrose concentrations. The lowest growth index was calculated for roots growing at the presence of $50 \mathrm{~g} / \mathrm{dm}^{3}$ of sucrose. In the further stage of the studies, the contents of iridoid and phenylethanoid glycosides were evaluated in methanol extracts of the hairy roots by UHPLC (Ultra High Performance Liquid Chromatography). It was found that several iridoids (harpagid, harpagoside and aucubin) and phenylethanoids (acteoside and isoacteside) were produced in the plant material. It was also observed that the levels of the compounds differed in tested extracts depending on the sucrose concentration in the growth medium.

\section{References}

[1] Lloyd G., McCown B. (1980) Int. Plant Propag. Soc. 30: 421-427.

[2] Oshio H. Inouye H. (1981) Phytochemistry 21: 133.

[3] Pharmacopoeia Commission of the People's Republic of China (2015) The pharmacopoeia of the People's Republic of China. Vol. 1. Chemical Industry Publishing House, Beijing.

[4] Zhang R.X., Li M.X., Jia Z.P. (2008) J. Ethnopharmacol. 117: 199-214. doi: 10.1016/j.jep.2008.02.018. 


\title{
Analysis of secondary metabolites in roots of in vitro-grown Gentiana cruciata, $G$. tibetica and their somatic hybrids
}

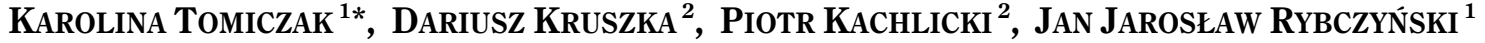 \\ ${ }^{1}$ Laboratory of Plant Biotechnology and Cryobiology, Department of Plant Experimental Biology, \\ Polish Academy of Sciences Botanical Garden - Center for Biological Diversity Conservation in Powsin, Poland \\ ${ }^{2}$ Department of Pathogen Genetics and Plant Resistance, Institute of Plant Genetics, Polish Academy of Sciences, Poznań, Poland \\ *k.tomiczak@obpan.pl
}

Plants belonging to the genus Gentiana are well known for their pharmaceutical values. These medicinal properties result from the synthesis of numerous biologically active secondary metabolites like secoiridoids, alkaloids, xanthones, flavonoids, steroles and anthocyanins. Secoiridoids, such as the most popular gentiopicroside, swertiamarine or amarogentin, are responsible for the bitterness of gentians and have gastroprotective, hepatoprotective, anti-inflammatory, antibacterial, and antitumor activity. Xanthones, like mangiferin or gentisin, are antidepressants and have antimicrobial, antioxidant, cardiotonic, and tuberculostatic effects [1,2].

Gentiana cruciata L. (Cross Gentian) and Gentiana tibetica King (Tibetan Gentian) are two endangered tetraploid gentian species of great medicinal importance. Gentiana cruciata is widespread in most of Europe and in Western Asia, but it is nowhere very common. Gentiana tibetica grows in western Nepal, Buthan and south-eastern Tibet, where is an important herbaceous plant in traditional medicine. In order to create cells with new genetic constitution, and consequently, with unique profiles of secondary metabolites, these two species have been artificially hybridized via protoplast electrofusion [3]. The obtained somatic hybrid plants grew vigorously and easily rooted in vitro. The aim of this work was to compare the concentration of gentiopicroside, swertiamarine and mangiferin in roots of selected in vitro-grown somatic hybrids and their parental species. The quantitative analysis was performed by ultra-performance liquid chromatography (UPLC) with the photodiode array detector (PDA). Additionally, a qualitative analysis of other secondary metabolites of $G$. cruciata and $G$. tibetica was carried out using high performance liquid chromatography (HPLC)/ion-trap mass spectrometry (MS).

The results showed that roots of somatic hybrids $G$. cruciata $(+)$ G. tibetica contain significantly less gentiopicroside (from 1.57 to $3.86 \mathrm{mg} / \mathrm{g}$ dry weight) than the parental species (on average $5.01 \mathrm{mg}$ and $9.72 \mathrm{mg} / \mathrm{g}$ dry weight for G. cruciata and G. tibetica, respectively). In the case of swertiamarin and mangiferin, only the limit of detection was achieved. Moreover, some secoiridoids like gelidoside, trifloroside, or derivatives of sweroside, as well as some valuable flavonoids (derivatives of isoorientin and isowitexin) were identified in the roots of G. cruciata and G. tibetica. The occurrence of these compounds has never been reported for these species before.

\section{References}

[1] Drobyk N.M., Mel'nyk V.M., Twardovska M.O., Konvalyuk I.I., Kunakh V.A. (2015) [In:] The Gentianaceae - Vol. 2: Biotechnology and Applications. Eds. Rybczyński J.J., Davey M., Mikuła A. Springer, Berlin-Heidelberg: 307-317.

[2] Mirzaee FA., Hosseini A., Bakhshi Jouybari H., Davoodi A., Azadbakht M. (2017) J. Trad. Compl. Med. 7(4): 400-408.

[3] Tomiczak K., Mikuła A., Rybczyński J.J. (2015) [In:] The Gentianaceae - Vol. 2: Biotechnology and Applications. Eds. Rybczyński J.J., Davey M., Mikuła A. Springer, Berlin-Heidelberg: 163-185. 


\title{
Poster no. 48
}

\section{The influence of $\mathrm{NaCl}$ and melatonin on Amaryllidaceae alkaloids biosynthesis}

\author{
Agata PtaK $^{1 *}$, Magdalena Simlat ${ }^{1}$, Emilia Morańska ${ }^{1}$, Dominique Laurain-MatTaR ${ }^{2}$ \\ ${ }^{1}$ Department of Plant Breeding and Seed Science, University of Agricultural in Krakow, Poland \\ ${ }^{2}$ Universite de Lorraine, SRSMC, Vandœuvre-les-Nancy, France \\ *mfptak@cyf-kr.edu.pl; Dominique.Mattar@univ-lorraine.fr
}

Leucojum aestivum L. (summer snowflake) produces pharmacologically active Amaryllidaceae alkaloids: galanthamine and lycorine. Galanthamine is used for Alzheimer's disease treatment as an acetylcholinesterase inhibitor, while lycorine has antiviral and antimalarial properties. Moreover, it may inhibit cell division. Currently, clinical trials are carried out to determine the use of galantamine in the treatment of cancer. Nowadays there are two ways of a wide-scale galanthamine production: a total chemical synthesis and the extraction from field-grown plants, such as: Narcissus sp., Leucojum aestivum, Lycoris radiata, Ungernia victoria. Both of them have considerable disadvantages, such as low effectiveness and a relatively high cost. The biotechnological approach to obtaining galanthamine can be considered as an alternative way for the production of this alkaloids. However, the use of in vitro systems for galanthamine production, still needs to be improved. It is known that the efficiency of alkaloid biosynthesis in in vitro cultures is affected by biological, chemical, and physical factors, including different environmental stresses often referred to as elicitors. The results of our previous studies have shown that elicitation of $L$. aestivum plant cultures with methyl jasmonate and salicylic acid stimulates biosynthesis of galanthamine and lycorine [1].

In the present research the influence of $\mathrm{NaCl}(50,100,150$ and $200 \mathrm{mM})$, melatonin $(1,5,10 \mu \mathrm{M})$ and $\mathrm{NaCl}$ $(200 \mathrm{mM})$ in combination with melatonin $(1,5,10 \mu \mathrm{M})$ on the accumulation of Amaryllidaceae alkaloids in in vitro L. aestivum cultures was tested. Alkaloid extracts were analyzed by gas chromatography-mass spectrometry, which allowed to quantify galanthamine and lycorine and to identify other alkaloids.

The plants of $L$. aestivum cultivated on the medium enriched with $10 \mu \mathrm{M}$ of melatonin were characterized by highest biomass increments $(0.81 \mathrm{~g} \mathrm{FW})$. The lowest biomass increments were observed in the plants grown on the medium contained $200 \mathrm{mM}$ of $\mathrm{NaCl}(0.16 \mathrm{~g} \mathrm{FW})$. Moreover, the plants obtained under these conditions showed a tendency to turn brown and die. Galanthamine and lycorine were detected in all the plant cultures. However, the highest concentration of galanthamine was found in plants cultured on the medium supplemented with $5 \mu \mathrm{M}$ of melatonin $\left(46.885 \mu \mathrm{g} / \mathrm{dm}^{3}\right)$. The observed contents were about 58.7 times higher than in control plants. The highest lycorine concentration $\left(116.435 \mu \mathrm{g} / \mathrm{dm}^{3}\right)$ was found in plants grown on the medium with the addition of $10 \mu \mathrm{M}$ of melatonin. In that case the lycorine content was about 1.5 times higher as compared to control plants. The addition of $\mathrm{NaCl}$ to the medium did not have a favorable effect on galanthamine or lycorine biosynthesis. Six alkaloids were identified in L. aestivum in vitro plants. Melatonin led to the highest diversity of alkaloids in tissue culture extracts.

The obtained results indicate that melatonin may stimulate $L$. aestivum in vitro plants grow and Amaryllidaceae alkaloids biosynthesis.

\section{Acknowledgements}

The research was financed by the Ministry of Science and Higher Education of the Republic of Poland.

\section{References}

[1] Ptak A., Morańska E., Saliba S., Zieliński A., Simlat M., Laurain-Mattar D. (2017) Plant Cell Tiss. Org. Cult. 128: $335-345$. 
Poster no. 49

\title{
Biochemical characterization of Chelidonium majus in vitro cultures
}

\author{
MAREK NiEWIAdOMSKi ${ }^{1}$, KATARZYNa KOSTYRKa ${ }^{1 *}$, SABina ChMARA ${ }^{1}$,

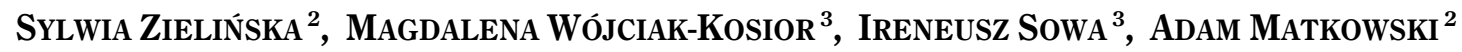 \\ ${ }^{1}$ Student Scientific Club no K 76, Department of Pharmaceutical Biology, Wroclaw Medical University, Poland \\ ${ }^{2}$ Department of Pharmaceutical Biology, Wroclaw Medical University, Poland \\ ${ }^{3}$ Department of Analytical Chemistry, Medical University of Lublin, Poland \\ *marek.niewiadomskii@gmail.com; sylwia.zielinska@umed.wroc.pl; kosiorma@wp.pl
}

Chelidonium majus L. (Papaveraceae) is a perennial species that prefers a nitrogen-rich soil. Of the two recognized species, $C$. majus is the only one that naturally occurs in Europe, parts of Asia and North America. The bioactive compounds present in this plant are isoquinoline alkaloids: benzophenanthridine derivatives (chelidonine, chelerythrine, sanguinarine), protoberberine, and protopine derivatives. The aim of this experiment was to introduce plants from this species into in vitro conditions and to check the impact of the culture conditions on the shoot and undifferentiated growth and development of cells, as well as the alkaloid accumulation. Aseptically obtained seedlings were used as a source of explants for the initiation of the cultures. Excised apical buds were placed on a proliferation medium. In order to induce callus tissue, explants from cotyledons, leaves, petioles and roots were used. The proliferation media consisted of B5, MS, and $1 / 2$ MS containing $3 \%$ or $1.5 \%$ of sucrose, and were optionally supplemented with a double amount of $\mathrm{NH}_{4}+$ ions and a simultaneous depletion of an equivalent amount of nitrate ions. MS proliferation media for the callus tissue induction and organogenic response were supplemented with plant growth regulators: three cytokinines: 6-benzylaminopurine - BA, kinetin - KIN, or thidiazuron - TDZ, and two auxins: 1-naphthaleneacetic acid - NAA, and 2,4-dichlorophenoxyacetic acid - 2.4-D. Shoots grown on eight different culture media differed significantly in terms of the number of nodes, the number and leaf blade size, and the number and the length of adventitious roots. The best explants for dedifferentiation and development of the callus were roots and leaves. In white light, an intense rhizogenesis appeared in the tissue cultured on the medium with 2,4-D + NAA + BA and 2,4-D + NAA + KIN. After the intersection of shoots, petioles and roots, milk juice (latex) with an intense red-orange color appeared, which indicated the prevailing sanguinarine content. The alkaloid composition was carried out using a VWR Hitachi Chromaster 600 chromatograph (Merck, Darmstadt, Germany) with a spectrophotometric detector (DAD) and EZChrom Elite software (Merck). Additionally, the chromatographic fractions eluted at the retention time characteristic for the investigated alkaloids were collected using a Foxy R1 fraction collector (Teledyne Isco, Lincoln, USA), and their identity was confirmed by a direct injection mass spectrometry (micrOTOF-Q II, Bruker Daltonics, Bremen, Germany) using Compass DataAnalysis software version 4.1. The quantitative analysis of quaternary alkaloids showed the presence of three classes of alkaloids, namely benzophenanthridine, protoberberine and protopine. Large differences were observed between shoots, roots and undifferentiated cell obtained in vitro. Coptisine was the most abundant compound in shoots, while sanguinarine and chelerythrine in roots. The callus was rich in protopine and sanguinarine. The methodology that has been developed in in vitro cultures will be used to optimize the composition of alkaloids and to study the influence of stress factors on the metabolic profile and antimicrobial bioactivity. 


\title{
Poster no. 50
}

\section{The relationship between the content of bioactive constituents and the sex of in vitro regenerated plantlets - the phytochemical analysis of dioecious species Rumex thyrsiflorus (Fingerh.)}

\author{
Katarzyna Dziedzic $^{1 *}$, Agnieszka Szopa ${ }^{2}$, Piotr Waligórski ${ }^{3}$, Halina EkierT $^{2}$, Halina ŚlesaK $^{1}$ \\ ${ }^{1}$ Department of Plant Cytology and Embryology, Jagiellonian University, Kraków, Poland \\ ${ }^{2}$ Department of Pharmaceutical Botany, Jagiellonian University Medical College, Kraków, Poland \\ ${ }^{3}$ The Franciszek Gorski Institute of Plant Physiology, Polish Academy of Sciences, Kraków, Poland \\ *katarzyna.dziedzic@doctoral.uj.edu.pl; a.szopa@uj.edu.pl; p.waligorski@ifr-pan.edu.pl
}

Rumex thyrsiflorus (Fingerh.) - pyramidal sorrel, thyrse sorrel, is a model dioecious plant species with heteromorphic sex chromosomes. The chromosome constitution of females is $2 \mathrm{n}=12 \mathrm{~A}+\mathrm{XX}$ and of males is $2 \mathrm{n}=12 \mathrm{~A}$ $+\mathrm{XY} 1 \mathrm{Y} 2$. This species is an attractive subject for studies on the evolution, structure and function of the polymorphic sex chromosomes system, the sex chromatin as well as the biased sex ratios observed at different stages of plant development in wild populations. Furthermore, extracts from the plant raw material of $R$. thyrsiflorus exhibit, for example haemostatic, anti - inflammatory, antiscorbutic, antiseptic and antiproliferative for human cancer cells activities. These biological activities are responsible various groups of bioactive substances, e.g.: flavonoids, phenolic acids, tanning agents, anthraquinones, proanthocyanidins and polyunsaturated fatty acids.

The aim of the conducted research was to analyze the quantitative and qualitative differences in the contents of phenolic acids (both, free and bound), catechins, and flavonoids between male and female plants regenerated under plant tissue cultures conditions.

The protocol of an efficient micropropagation was developed on Murashige and Skoog (MS) agar medium supplemented with $2.27 \mu \mathrm{M}$ thidiazuron (TDZ). The hypocotyls isolated from 11-day old sterile seedlings were used as the explants. The adventitious shoots formation via callus (indirect organogenesis) was observed during the regeneration.

To confirm the sex of the regenerated plantlets, PCR-based methods were used, which involved DNA markers located on Y chromosomes. The following pairs of primers were exploited: RAY - F (5'-ACT CGA ATG TAA GCA TTT GGT CCT A-3') and RAY - R (5'-ACT ACA CGA TTG TCC ATA AAG TGG A-3') to amplify male - specific sequence RAYI; UGR08 - F (5'-CCA ATT GGT CTC AAC TAG AAC A-3') and UGR08 - R (5'-TGT TAT AGG TTT TGG ACT GCC A-3') to amplify the male-specific repetitive sequence RAYII.

Additionally, to verify the template DNA quality, the amplification of RAE730 repetitive sequence located on the autosomes of $R$. thyrsiflorus, was carried out. For this purpose, the following primers were used: R730 - A ( $5^{\prime}-\mathrm{CTC}$ GGA CCA ATT ATC TCA T-3') and R730 - B (5'-CAT TAT TTG GGA GCC GAT-3').

Moreover, the qualitative evaluation of phenolic compounds was performed by the LC-DAD method. In the methanolic extracts from the studied micropropagated plants, acclimated ex vitro and growing in field conditions, 9 free phenolic acids (caffeic, chlorogenic, p - coumaric, cryptochlorogenic, ferulic, gallic, protocatechuic, neochlorogenic, vanillic), 9 flavonoids (apigenin, hyperoside, isoquercetin, myricetin, quercetin, quercitrin, rhamnetin, rutin, vitexin) and one catechin were confirmed. Additionally, in the methanolic extracts after acid hydrolysis two phenolic acids: p - hydroxybenzoic, syringic, beside caffeic, gallic, protocatechuic and vanillic were detected. The quantitative differences in the content of the phenolic compounds in male and female plants of $R$. thyrsiflorus regenerated in vitro were noticed, but a detailed elaboration of results is in progress. 
Poster no. 51

\title{
Echium plantagineum $\mathrm{L}$. hairy root cultures for the production of anticancer shikonin derivatives
}

\author{
Maegorzata Jeziorek ${ }^{1 *}$, Paulina Mazur ${ }^{1}$, Selin Gürcü ${ }^{2}$, Adrian Kasztelan ${ }^{1}$, \\ Katarzyna Sykeowska-BaraneK ${ }^{1}$, Agnieszka Pietrosiuk ${ }^{1}$ \\ ${ }^{1}$ Department of Pharmaceutical Biology and Medicinal Plants Biotechnology, \\ Faculty of Pharmacy with the Laboratory Medicine Division, Medical University of Warsaw, Poland \\ ${ }^{2}$ Ege University, Izmir, Turkey \\ *mjeziorek@wum.edu.pl
}

Echium plantagineum L. naturally occurs in many places all over the world including Europe, Australia and South America and is nowadays also often cultivated as an ornamental plant. The chemical profile of secondary metabolites for plants of this species collected in Australia confirmed the presence of pyrrolizidine alkaloids and shikonin derivatives, both characteristic for the Boraginaceae family [1]. Publications on the biotechnological research into Echium $s p$. are rare and include in vitro cultures of callus tissue, hairy roots and suspension cultures. Up to date studies of hairy roots have been conducted for $E$. acanthocarpum, demonstrating the presence of polyunsaturated fatty acids [2] and for $E$. rauwolfii dedicated to intensifying the production of pyrrolizidine alkaloids [3].

The aim of our study was to investigate the potential of $E$. plantagineum hairy roots for the production of shikonin derivatives such as acetylshikonin (ACS) and isobutyrylshikonin (IBS), known also for their anticancer potential [4]. The genetic transformation of E. plantagineum L. was performed using Agrobacterium rhizogenes ATCC 15834 as a vector. Four hairy root lines were obtained in order to produce red pigments and the genetic transformation for all examined root lines (TR1, TR2, TR6 and TR7) was confirmed using the PCR technique. The TR6 line was selected for further studies as showing the greatest potential for shikonin derivatives production. Further, the potential of DCR [5], WPM [6], B5 [7] and SH [8] media for the growth and production enhancement was tested. TR6 line showed a significant growth and shikonin derivatives production in all media used. Supplementing the media with $50 \mathrm{~g} / \mathrm{dm}^{3}$ sucrose highly potentiated root growth and metabolites production. The presence of 7 shikonin derivatives in root tissues and in post-culture media hexane extracts was demonstrated using the DAD HPLC technique. Among them the ACS and IBS presence was confirmed using standard substances by TLC and HPLC DAD. ACS predominated over IBS in the root extracts and in post-culture media extracts. Shikonin traces were found only in very few samples.

Our studies report for the first time the presence of ACS and IBS as shikonin derivatives in in vitro culture of E. plantagineum L. hairy roots and show its promising potential for the enhanced production of the biologically active naphthazarines.

\section{References}

[1] Weston P.A., Weston L.A., Hildebrand S. (2013) Phytochem. Rev. 12(4): 831-837.

[2] Cequier-Sánchez E., Rodríguez C., Dorta-Guerra R., Ravelo A.G., Zárate R. (2011) BMC Biotechnol. 11(1): 42.

[3] El-Mawla A. (2010) Orig. Artic. 65: 224-226.

[4] Jeziorek M., Sykłowska-Baranek K., Pietrosiuk A. (2017) Curr. Med. Chem. 24: 1-22.

[5] Gupta P.K., Durzan D.J. (1985) Plant Cell Rep. 4: 177-179.

[6] Lloyd G., McCown B. (1980) Comb. Proc. Int. Plant Propag. Soc. 30: 421-427.

[7] Gamborg O.L., Miller R.A., Ojima K. (1968) Exp. Cell Res. 50(1): 151-158.

[8] Schenk R.O.Y.U., Hildebrandt A.C. (1972) Can. J. Bot. 50: 199-204. 


\title{
Poster no. 52
}

\section{The impact of plant growth regulators on the accumulation of pharmaceutically important secondary metabolites in Vitex rotundifolia shoot cultures}

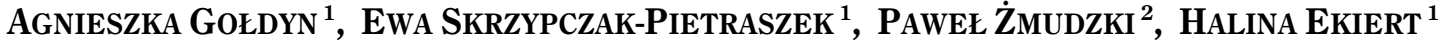 \\ ${ }^{1}$ Chair and Department of Pharmaceutical Botany, Faculty of Pharmacy, Collegium Medicum, Jagiellonian University, Kraków, Poland \\ ${ }^{2}$ Chair of Pharmaceutical Chemistry, Faculty of Pharmacy, Collegium Medicum, Jagiellonian University, Kraków, Poland
}

Vitex agnus castus is the best known medicinal species from the genus Vitex and its fruits has been devoted a monograph in the European Pharmacopoeia $9^{\text {th }}$ Edition. Vitex rotundifolia is an Asian species poorly known in Europe [1].

Iridoids, flavonoids and phenolic acids are important groups of plant secondary metabolites with different, valuable therapeutic properties (e.g. antiphlogistic, antioxidant, antiseptic) [2].

The aim of the study was to test the influence of five variants of Murashige and Skoog (MS) medium [3] supplemented with different combinations of plant growth regulators (PGRs) on the accumulation of iridoids (agnuside, aucubin), flavonoids and phenolic acids in Vitex rotundifolia L. (Lamiaceae) shoot cultures.

In vitro cultures were initiated from tops of $V$. rotundifolia shoots (plants from the greenhouse - Garden of Medicinal Plants, Jagiellonian University, Medical College, Cracow) on MS medium supplemented with BAP - $1.0 \mathrm{mg} / \mathrm{dm}^{3}$, $\mathrm{NAA}-0.5 \mathrm{mg} / \mathrm{dm}^{3}$ and $\mathrm{GA}_{3}-0.25 \mathrm{mg} / \mathrm{dm}^{3}$. Numerous shoots appeared after 3-4 weeks from initiation and shoot cultures were obtained. Five variants of MS medium supplemented with different combinations of PGRs were tested: A) BAP $-2.0 \mathrm{mg} / \mathrm{dm}^{3}$, NAA - $1.0 \mathrm{mg} / \mathrm{dm}^{3}$; B) BAP $-1.0 \mathrm{mg} / \mathrm{dm}^{3}$, NAA $-0.5 \mathrm{mg} / \mathrm{dm}^{3}, \mathrm{GA}_{3}-0.25 \mathrm{mg} / \mathrm{dm}^{3}$; C) BAP - $2.0 \mathrm{mg} / \mathrm{dm}^{3}$, NAA - $2.0 \mathrm{mg} / \mathrm{dm}^{3}$; D) BAP - $2.0 \mathrm{mg} / \mathrm{dm}^{3}$, NAA $-0.2 \mathrm{mg} / \mathrm{dm}^{3}$; E) BAP - $3.0 \mathrm{mg} / \mathrm{dm}^{3}$, NAA $-1.0 \mathrm{mg} / \mathrm{dm}^{3}$. V. rotundifolia shoots (inoculum $-1 \mathrm{~g}$ ) were transferred to Erlenmeyer flasks ( $300 \mathrm{ml}$ ) containing five variants of MS medium (100 ml of medium, 5 flasks per variant). The biomass was harvested after 6 weeks, dried, weighted and used for phytochemical analyses. Qualitative and quantitative HPLC-DAD analyses of flavonoids, phenolic acids and UPLC-MS/MS of iridoids in methanolic extracts from the biomass were conducted [4].

Two iridoids (agnuside, aucubin), five flavonoids (apigenin, casticin, luteolin, quercetin, vitexin), five phenolic acids (3,4-dihydroxyphenylacetic, neochlorogenic, $p$-hydroxybenzoic, protocatechuic and vanillic acids) were identified and quantified. The contents of the compounds were varied depending on the variant of the medium. The main compounds were: agnuside, vitexin, neochlorogenic and $p$-hydroxybenzoic acids (up to about 960, 430, 320 and $110 \mathrm{mg} / 100 \mathrm{~g}$ DW, respectively). The contents of aucubin and quercetin did not exceed $50 \mathrm{mg} / 100 \mathrm{~g} \mathrm{DW}$; the contents of apigenin, luteolin, casticin and protocatechuic, 3,4- ihydroxyphenylacetic and vanillic acids were below $20 \mathrm{mg} / 100 \mathrm{~g}$ DW. The best growth and production medium appeared to be variant (D).

There are only few publications on the production of secondary metabolites in in vitro cultures of plants from the genus Vitex and our results are the first concerning V. rotundifolia.

\section{References}

[1] Rani A., Sharma A. (2013) Phcog. Rev. 7: 188-198.

[2] Khadem S., Marles R. (2010) Molecules 15: 7985--8005.

[3] Murashige T., Skoog F. (1962) Physiol. Plant. 15: 473-497.

[4] Ellnain-Wojtaszek M., Zgórka G. (1999) J. Liq. Chrom. Rel. Technol. 22: 1457-1471. 
Poster no. 53

\title{
Sarracenia alata microcuttings as a potential source of biologically active substances
}

\author{
Barteomiej Skalny ${ }^{1}$, Renata GaleK ${ }^{1 *}$, Antoni Szumny ${ }^{2}$, JACEK ŁyCZKo $^{2}$ \\ ${ }^{1}$ Department of Genetics, Plant Breeding and Seed Production, University of Environmental and Life Sciences, Wrocław, Poland \\ ${ }^{2}$ Department of Chemistry, University of Environmental and Life Sciences, Wrocław, Poland \\ * renata.galek@upwr.edu.pl
}

Sarraceniaceae is a New World carnivorous plant family comprising three genera: Darlingtonia Torr. (monotypic), Heliamphora Benth. (ca. 23 species) and Sarracenia L. (ca. 11 species). The pale pitcher plant-yellow trumpets (Sarracenia alata) are one of the eleven species belonging to the family Sarraceniaceae which occupies in North America. Sarracenia purpurea and $S$. flava have been most studied in terms of metabolite profiling and their medical usefulness. These carnivorous plants were traditionally used by many aboriginal communities as a medicinal plant against a wide spectrum of diseases including tuberculosis. Various compounds found in Sarracenia have been reported, including volatiles, flavonoids, phytochemicals and pitcher fluid composition. Chemical constituents (especially volatiles) were applied to study the similarities among different species of Sarraceniaceae phylogeny. Sarracenia alata is relatively poorly described in terms of chemical constituents. Additionally knowledge about of microcutings possibility possessing is limited, because of fact that micropropagation takes place mainly in private companies. The aim of current study was to elaborate the protocol for micropropagation of $S$. alata and to analyze the particular chemical composition of volatile compounds of the studied plant. The initial material consisted of single trimmed plants $(2.53 .0 \mathrm{~cm}$ length). The effect of 6-benzylaminopurine (BAP) concentration $(0.5,1.0,1.5,2.0$, $\left.2.5,3.00 .0 \mathrm{mg} \cdot \mathrm{dm}^{-3}\right)$ with IAA combinations $\left(0.3 \mathrm{mg} \cdot \mathrm{dm}^{-3}\right)$ was tested as the first step of the experiments. MS was used as the basal medium with a reduced content of macro and micronutrients to $1 / 3$. In the second phase of experiment explants earlier obtained were first measured and then cut $(2.5-3.0 \mathrm{~cm}$ length), separated on the single plants and placed on 1/3 MS. They were cultivated for the next 14 weeks. Such features as the length of the plants, the number of leaves, new shoots, roots length $[\mathrm{cm}]$, the number of roots, plant fresh and dry weight $[\mathrm{g}]$ were assessed after 12 and 14 weeks. The new shoots and leaves proliferation were most effective on media supplemented in higher doses of BAP (1.5 up to 3.0 in combination with 0.3 IAA) and the number of new shoots ranged between $5-6$ with 25 leaves in the first phase of experiment. Similarly suitable results were noted at the second phase of the experiment in the case where plants were cultivated earlier on the previously mentioned medium. They produced on average four new shoots with 22 leaves. All subcultured shoots developed root system after 12 or the next 14 weeks of culture. The best plant rooting process was observed on 1/3 MS medium and 1/3 MS supplemented with 0.3 IAA (average 4 roots, length above $1 \mathrm{~cm}$ ). The media containing a higher concentration of BAP above $1.0 \mathrm{mg} \cdot \mathrm{dm}^{-3}$ in combination with 0.3 IAA had a significant influence on the greatest fresh and dry mass of plants. The volatile compounds presented in plant-yellow trumpets were analyzed using the solid-phase microextraction (SPME) approach on GC-MS Varian 2000/2000 apparatus. Several linear short-chain alcohols as well as aldehydes were found, e.g. 2,4-hexadien-1-ol; 2-hexenal, (E); 2-hexen-1-ol, (Z)-; 3-hexen-1-ol, acetate, (Z)-. The predominated sesquiterpenoid compounds were caryophyllene and $\alpha$-bergamotene. In volatiles the diterpenoid labda-8(20),12,14triene as well their isomers were identified. Some of the compounds found in $S$. alata could play a main role as attractants of insects. 


\title{
Poster no. 54
}

\section{The content of anthocyanins in potato plants in in vivo and in vitro cultures}

\author{
JÓZEF KLOCEK ${ }^{1 *}$, GUY COSTA ${ }^{2}$, MARCIN HORBOWICZ ${ }^{1}$ \\ ${ }^{1}$ Department of Botany and Plant Physiology, University of Podlasie, Siedlce, Poland \\ ${ }^{2}$ Laboratoire de Chimie des Substances Naturelles (LCSN EA 1069), Faculté des Sciences et Techniques, \\ Universitie de Limoges, France \\ *klocek@ap.siedlce.pl; guy.costa@unilim.fr
}

A number of plants produces anthocyanin compounds in in vitro cultures of cells, tissues and organs. Their list includes about 30 species. The most commonly used anthocyanin producers are: Daucus carota, Vitis vinifera, Perilla frutescens, Aralia cordata and Fragaria pineapple[1]. Increasingly, there are also potato varieties with varying levels of anthocyanins and attempts are being made to market them on the domestic market. The content of anthocyanins in plants of two potato varieties was examined. They were: 1) Bleue Fleur (introduced to trade, the country of origin: France), 2) Irga (widely used in households, the country of origin Poland) growing in in vitro and in vivo cultures under the conditions of breeding rooms at the Department of Botany and Physiology Plants UPH in Siedlce.

Assays for determining anthocyanins were collected weekly from the apical leaves of plants. Determinations of the anthocyanin content were made according to the modified method of Mancinelli et al. (1988) [2]. The extraction was carried out with $1 \% \mathrm{HCl}$ in methanol in the dark for 24 hours. The content of anthocyanins was converted into a cyanidin-3-glycoside.

Different amounts of anthocyanins were observed in the studied varieties. In plants of the Bleue Fleur cultivar, a high content of anthocyanins was observed in vivo followed by its gradual decrease and in plants growing in vitro, the initial anthocyanin content was low and only increased in the last trial. In plants of the cultivar Irga growing in vivo, first the high content of anthocyanins was observed, and then followed its gradual decrease, while in plants growing in vitro, also at the beginning of the research, the anthocyanin content was higher and then decreased. The varieties also differed in the anthocyanin content throughout the study period. On average, they contained slightly more Irga plants than Blue Fleur in in vivo culture and in the in vitro culture of Bleue Fleur. Thus, the potato varieties differed in the content of anthocyanins. Also, the in vivo or in vitro culture type influenced the content of these compounds in the studied plants. The Polish potato variety Irga in vivo had a slightly higher content of anthocyanins than the French variety with blue tubers Bleue Fleur.

In conclusion, we may state that the Polish potato variety Irga was not doing worse that the foreign variety regarding the content of anthocyanins.

\section{References}

[1] Grajek W. (2001) Food Chem. Toxicol. 69: 1667-1861.

[2] Mancinnelli A.L. (1988) Plant Physiol. 86: 749-753. 


\section{List of species}

A

Abelmoschus esculentus /okra Acalypha indica

Aconitum bucoviniense

Agrobacterium rhizogenes

Agrobacterium sp.

Agrobacterium tumefaciens

Allium sativum

Alyssum montanum

Arabidopsis thaliana 232, 252, 271, 272, 279, 315

Aralia cordata

Arnica montana

Avena fatua

Avena sativa /oat

B

Beta vulgaris / sugar beet

Brassica napus

Brassica oleracea

Brassica rapa

C

Camellia sinensis

Candida albicans

Cannabis sativa

Capsicum annuum

Carex buxbaumii

Carpesium divaricatum

Ceratopteris thalictroides

Chelidonium majus

Chrysanthemum $\times$ grandiflorum

Cicer arietinum

Cinnamomum altissimum

Citrus limon

Citrus sinensis

Codonopsis pilosula

Codonopsis tansheng

Coffea sp.

Cucumis melo

Cucumis sativus

Cucurbita maxima

Cucurbita moschata

Cucurbita pepo

Cyathea delgadii

Cycas sp.

D

Daphne mezereum

Darlingtonia sp.

Daucus carota /carrot

252

296

321

226,234

$226,270,278,281$

276

306

324

268

252

241, 244, 289

291, 308

$266,283,290$

266,274

266

227

264

299

227

268

264

270

319

236, 237

275

227

227

232

303

303

227

278

265

278

278

278

269,313

227

253

323

281, 286, 324
Dimocarpus longan

232

Dionaea muscipula

227, 312

Drosera peltata

312
E

Echium acanthocarpum 321

Echium plantagineum 321

Echium rauwolfii $\quad 321$

Embelia ribes 294

Eucalyptus angophoroides $\quad 227$

$\mathbf{F}$

Fagopyrum esculentum 255

Fallopia aubertii 228

Fragaria x ananasa $\quad 277$

Fragaria pineapple 324

Fusarium sp. 246, 256

\section{G}

Gentiana cruciata 317

Gentiana tibetica $\quad 317$

Gerbera jamesonii $\quad 309$

Glomus intraradices $\quad 257$

Glycine max/soyabean 230, 273

Gossypium hirsutum 232

H

Hebeloma crustuliniforme 257

Heliamphora sp. 323

Helodium blandowii 248

Hibiscus cannabinus $\quad 227$

Hordeum vulgare /barley 230, 234, 245, 288, 315

Huperzia pinifolia 267

Huperzia selago $\quad 267$

Hypericum perforatum 226

I

Inula sp.

264

$\mathbf{L}$

Lactuca sativa $\quad 252$

Lagenaria siceraria $\quad 278$

Lamprocapnos spectabilis $\quad 260$

Larix kaempferi 232

Larix leptolepis 232

Lathyrus cicera $\quad 275$

Lathyrus sativus $\quad 259$

Lens culinaris $\quad 275$

Leucojum aestivum $\quad 318$

Linnaea borealis 263 
Linum usitatissimum /flax

$233,238,256,302$,

305,314

278

Luffa cylindrical

Lupinus albus

Lupinus angustigolius

Lupinus mutabilis

Lycopodiella inundata

Lycopodium sp.

Lycoris radiata

\section{M}

Magnolia acuminata $\times M$. brooklynensis

Magnolia acuminata $\times M$. denudata

Magnolia grandiflora

Malus sp.

Manihot esculenta

Medicago truncatula

Miscanthus $\times$ giganteus

Miscanthus sinensis

Moluccella laevis

$\mathbf{N}$

Narcissus sp.

o

Oryza sativa / rice

$\mathbf{P}$

Paludella squarrosa

Paphiopedilum sp.

Paxillus involutus

Perilla frutescens

Perovskia abrotanoides

Perovskia atriplicifolia

Phaseolus vulgaris /common bean

Phlegmariurus squarrosus

Pinus sylvestris

Pisum sativum

Populus $\times$ canescens

Populus nigra

Prunus cerasifera

Prunus cerasus $\times P$. canescens

Prunus domestica

Pteris vittata

Pulsatilla patens

Pulsatilla pratensis

\section{$\mathbf{R}$}

Ranunculus illyricus

Rehmannia elata

Rehmannia glutinosa

Rhizophagus irregularis

Ribes grossularia

$230,235,252$

261,316

261

257

294
Ribes magellanicum $\quad 294$

Rindera graeca 262

Rosa rugosa $\quad 227$

Rubus sp. $\quad 294$

Rumex sp. 243

Rumex thyrsiflorus $\quad 320$

$\mathbf{S}$

Salix lapponum 249

Salix myrtilloides $\quad 249$

Salvia bulleyana $\quad 300$

Salvia miltiorrhiza $\quad 300$

Sarracenia alata $\quad 323$

Sarracenia flava 323

Sarracenia purpurea $\quad 323$

Scorpidium scorpioides 248

Secale cereale 239, 292

Silene sp. 243

Silene vulgaris $\quad 307$

Solanum lycopersicum / tomato $\quad 252,273,284$

Solanum sisymbriifolium 284

Solanum tuberosum / potato 280, 324

Staphylea pinnata 251

Staphylococcus aureus $\quad 264$

Stevia rebaudiana $\quad 310$

$\mathbf{T}$

Taraxacum pieninicum 295

Telekia speciosa $\quad 264$

Thelypteris palustris 297

Tomenthypnum nitens 248

Triticosecale/triticale 239, 240, 242, 287

Triticum aestivum/wheat 230, 242, 246, 288, 298

$\mathbf{U}$

Ungernia victoria

318

V

Vaccinium corymbosum 301

Vaccinium sp. 301, 294

Vicia faba 275

Vicia narbonensis $\quad 275$

Viola epipsila $\quad 268$

Vitex agnus-castus $\quad 322$

Vitex rotundifolia $\quad 322$

Vitis vinifera 324

\section{$\mathbf{W}$}

Wisteria sinensis 228

\section{$\mathbf{z}$}

Zea mays /maize 232, 235, 241, 252, 257, 273, 289 


\section{List of authors and participants}

A

Adamczuk Aneta

Adamski Tadeusz

Adamus Adela

Ayres Phil

B

Bajor Magdalena

Banach-Albińska Barbara

Banasiuk Rafał

Barański Rafał

Barzyk Paweł

Berko Petr

Biesaga-Kościelniak Jolanta

Błażejewska Kamila

Boba Aleksandra

Bocian Karol

Bohater Aleksandra

Bojarczuk Krystyna

Broda Zbigniew

Burza Wojciech

C

Cegielska-Taras Teresa

Cerazy-Waliszewska Joanna

Chmara Sabina

Chmielewska Beata

Chylewska Agnieszka

Cichorz Sandra

Ciereszko Iwona

Cioć Monika

Ciszak Kamil

Costa Guy

Czyczyło-Mysza Ilona

Ćwiek-Kupczyńska Hanna

Ćwiklińska Paulina

D

Domnicz Beata

Dreger Mariola

Dubas Ewa

Dubert Franciszek

Dyduch-Siemińska Magdalena

Działo Magdalena

Dziedzic Katarzyna

Dziubańska Paulina

Dziurka Kinga

Dziurka Michał

E

Ekiert Halina

\begin{tabular}{|c|c|}
\hline & $\mathbf{F}$ \\
\hline 314 & Figas Anna \\
\hline 285 & Fikowicz-Krośko Jakub \\
\hline 274,282 & Florek Edyta \\
\hline 228 & $\mathbf{G}$ \\
\hline & Gaj Małgorzata Danuta \\
\hline 248 & Gajecka Monika \\
\hline 249 & Galek Renata \\
\hline 227,312 & Gasparis Sebastian \\
\hline 281 & Gawroński Jacek \\
\hline 285 & Gidzinska Weronika \\
\hline 265 & Gieniec Miron \\
\hline 298 & Gołdyn Agnieszka \\
\hline 278 & Gośka Maria \\
\hline 256,280 & Górecka Krystyna \\
\hline 283 & Górecka Mirosława \\
\hline 304 & Gregory Franklin \\
\hline 257 & Grzebelus Ewa \\
\hline 292 & Grzegorczyk-Karolak Izabela \\
\hline 265 & Grzyb Małgorzata \\
\hline & Gürcü Selin \\
\hline 266 & H \\
\hline 235 & Hamann Heiko \\
\hline 319 & Hanus-Fajerska Ewa \\
\hline 245 & Heinrich Mary K. \\
\hline 227 & Hnitecka Agata \\
\hline 291 & Hofstadler Daniel \\
\hline 314 & Horbowicz Marcin \\
\hline 251,309 & Hornyák Marta \\
\hline 288 & 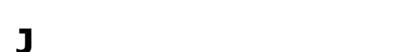 \\
\hline 324 & $J_{1}+1$ \\
\hline $241,244,289$ & Jacek Łyczko \\
\hline 285 & Janeczko Anna \\
\hline 248 & Jelonek Janusz \\
\hline & Jeziorek Małgorzata \\
\hline & Jeżowski Stanisław \\
\hline 274 & Joachimiak Andrzej \\
\hline 299 & Juzoń Katarzyna \\
\hline 287 & 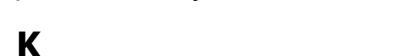 \\
\hline 255,298 & \\
\hline 310 & Kachlicki Piotr \\
\hline 238 & Kaczmarek Ewa \\
\hline 320 & Kaczmarek Zygmunt \\
\hline 248 & Kalisz Andrzej \\
\hline $241,244,289,298$ & Kała Maciej \\
\hline $255,289,298,312$ & Kamińska Monika \\
\hline & Kapłoniak Kamila \\
\hline & Karpiński Stanisław \\
\hline 320,322 & Kasztelan Adrian \\
\hline
\end{tabular}

232, 272, 279

282,323

230, 234

310

227

281

322

291

276

307

226

281, 286

300

247, 269, 313

321

228

306, 307

228

305

228

324

255

323

298

245

321

235

243

287

317

268

285

309

234

295

241, 244, 289

288, 308

321 
Kawka Mateusz

Kempa Michał

Kernbach Serge

Kępczyńska Ewa

Kępczyński Jan

Kieliszewska-Rokicka Barbara

Kiełkowska Agnieszka

Kistela Jolanta

Kistela Paweł

Kiszczak Waldemar

Klimek-Chodacka Magdalena

Klocek Józef

Kłeczek Natalia

Kocot Dawid

Kolniak-Ostek Joanna

Kopeć Przemysław

Kosieradzka Iwona

Kosiński Jan

Kostyn Kamil

Kostyrka Katarzyna

Koszelnik-Leszek Anna

Kościelniak Paulina

Kowalczyk Jan

Kowalska Monika

Kowalska Urszula

Kozak Bartosz

Kozak-Stankiewicz Kamila

Kozłowska Weronika

Koźmińska Aleksandra

Krela Rafał

Królicka Aleksandra

Kruszka Dariusz

Krychowiak Marta

Krzewska Monika

Kucharska Danuta

Kuczyńska Anetta

Kuksin Igor

Kulczyk-Skrzeszewska Magdalena

Kulińska Alicja

Kulma Anna

Kulpa Danuta

Kulus Dariusz

Kurasiak-Popowska Danuta

Kurowska Marzena

Kwiatkowski Wojciech

\section{$\mathbf{L}$}

Labudda Mateusz

Laurain-Mattar Dominique

Lema-Rumińska Justyna

Linkiewicz Anna

Łysienko Izabela

\section{M}

Maciejewska-Hoza Anna

Maćkowska Katarzyna

Makowczyńska Joanna

Makowski Wojciech

Malaga Sabina
258, 304
Malarz Janusz

Malicka Małgorzata 291

Malinowski Tadeusz 276

Mańko Katarzyna

Mańkowska Grażyna

262

Marasek-Ciołakowska Agnieszka

299, 302

Marcińska Izabela

273

Markiewicz Monika

$241,244,289$

Marzec Marek

277, 293

Matkowski Adam

Matusiewicz Magdalena

$258,304,319$

Mazur Paulina

225

321

Michalak Angelika $\quad 227$

Michalak Kornel M. $\quad 297$

Michałowska Emilia 236

Mikołajczak Krzysztof $\quad 285$

Mikołajczyk Sylwia

246,292

Mikuła Anna

$247,269,270,313$

Morańska Emilia

318

Mól Rafał

Muszyńska Ewa

307

$\mathbf{N}$

Nadolska-Orczyk Anna 230, 234

Narajczyk Magdalena $\quad 227$

Nielsen Stig $\quad 228$

Niemann Janetta 246

Niewiadomska-Wnuk Angelika 294

Niewiadomski Marek $\quad 319$

Noceń Joanna 308

Noga Angelika $\quad 241,244,289$

Nowak Katarzyna 279

Nowakowska Karolina 253

Nowicka Anna 287

$\mathbf{0}$

Ogrodowicz Piotr 285

Oleszczuk Sylwia 242

Oleszkiewicz Tomasz $\quad 281$

Olszak Marcin 265

Orczyk Wacław 230, 234

Ostrowska Agnieszka 298

Ozymko Zofia 248

\section{P}

Pacholczak Andrzej 253

Palenica Klaudia 248

Pałucka Małgorzata $\quad 268$

Parzymies Marzena 249, 311

Pastuszak Jakub 255

Pawłowska Bożena 251, 309

Piątczak Ewelina 261

Pietrosiuk Agnieszka 262, 267, 321

Pilarek Maciej 262

Piniak Magdalena $\quad 316$

Piosik Łukasz 284

Piwowarczyk Barbara 259, 312

Pluta Stanisław $\quad 301$

Płażek Agnieszka 255 
Pniewski Tomasz

Podwyszyńska Małgorzata 273

Pogorzelec Magdalena 249

Poniewozik Monika 311

Ponitka Aleksandra 235

Prokopiuk Barbara $\quad 251$

Przyborowski Mateusz 234

Ptak Agata 318

Pudelska Hanna

$\mathbf{R}$

Rokicki Michał

Radosz Joanna

Ruta-Piosik Maria

284

Rybczyński Jan Jarosław

$247,269,270,313,317$

\section{$\mathbf{S}$}

Sawicka-Sienkiewicz Ewa

Schmickl Thomas 228

Serafin Artur $\quad 249$

Siatkowski Idzi 246

Siegień Irena 314

Simlat Magdalena $\quad 318$

Sitarek Mirosław 273

Sitarski Adam 308

Siwek Julianna $\quad 281$

Siwik-Ziomek Anetta 283

Skalny Bartłomiej 323

Skała Ewa

Skrzypczak Tomasz

Skrzypczak-Pietraszek Ewa

Skrzypek Edyta

Słomka Aneta

Sobańska Karolina

Solarz Marta

Sosnowska Katarzyna

Sowa Ireneusz

Sowa Sławomir

Sowik Iwona

Stojakowska Anna

Stoy Kasper

Surma Maria

Sykłowska-Baranek Katarzyna

Szalata Milena

Szała Laurencja

Szarejko Iwona

Szczurowska Agnieszka

Szczygieł Krystyna

Szechyńska-Hebda Magdalena

Szewczyk-Taranek Bożena

Szklarczyk Marek

Szlachtowska Anna

Szopa Agnieszka

Szopa-Skórkowski Jan

Szot Paweł

Szumny Antoni

Szymański Tomasz

Szyp-Borowska Iwona

Szypuła Wojciech

Ślesak Halina

299,302

266,290

245,315

249

254

308

251,309

274

274

320

$233,238,256,280$

311

323

288

254

267

320
Ślusarkiewicz-Jarzina Aurelia

235,275

Swięcicki Wojciech 285

Świgoń Daria 227

Świstowska Alicja 249

$\mathbf{T}$

Taciak Magdalena

Thiem Barbara 263

Tokarz Krzysztof 259, 312

Tomaszewicz Wojciech 247, 313

Tomaszewska-Sowa Magdalena 283

Tomiczak Karolina

Tomkowiak Agnieszka

Trejgell Alina

$247,270,317$

246, 292

295

258

285

278

280

237

Tymoszuk Alicja

$\mathbf{U}$

Urbanowicz Oliwia

248

$\mathbf{W}$

Wadurkar Shraddha 228

Wahby Mostafa $\quad 228$

Wajdzik Marcelina 274

Waligórski Piotr 320

Warchoł Marzena 241, 244, 289

Weigt Dorota 246, 292

Wielgus Karolina 302

Wiszniewska Alina 306

Wiśniewski Kamil 258

Wojciechowicz Maria Katarzyna $\quad 275$

Wojciechowska Renata 312

Wojtania Agnieszka 277, 293

Wojtaszek Przemysław 228, 271

Wójciak-Kosior Magdalena 319

Wójcik Anna Maria

232, 279

272

308

259

303

Wróblewska Katarzyna

Wyłupek Izabela

Wysocka Marta

Wysokińska Halina

\section{Z}

Zahadat Payam

228

Zbieszczyk Justyna 245

Zdziechowska-Dudek Maria $\quad 288$

Zenkteler Elżbieta $\quad 284,297$

Zenkteler Maciej 284

Ziabka Magdalena $\quad 227$

Zielińska Sylwia $\quad 304,319$

Zieliński Kamil 287
Zimny Janusz

Zyprych-Walczak Joanna

229,239

246

322

Żmudzki Paweł

Żuk Magdalena

$233,238,305$

Żur Iwona

240, 287 